\title{
A comprehensive set of elemental abundances in damped Ly $\alpha$ systems: Revealing the nature of these high-redshift galaxies ${ }^{\star}$
}

\author{
M. Dessauges-Zavadsky ${ }^{1}$, F. Calura ${ }^{2}$, J. X. Prochaska ${ }^{3}$, S. D’Odorico ${ }^{4}$, and F. Matteucci², \\ 1 Observatoire de Genève, 51 Ch. des Maillettes, 1290 Sauverny, Switzerland \\ 2 Dipartimento di Astronomia-Universitá di Trieste, via G. B. Tiepolo 11, 34131 Trieste, Italy \\ ${ }^{3}$ UCO/Lick Observatory, University of California at Santa Cruz, Santa Cruz, CA 95064, USA \\ ${ }^{4}$ European Southern Observatory, Karl-Schwarzschildstr. 2, 85748 Garching bei München, Germany \\ 5 INAF, Osservatorio Astronomico di Trieste, via G. B. Tiepolo 11, 34131 Trieste, Italy
}

Received 3 September 2003 / Accepted 26 November 2003

\begin{abstract}
By combining our UVES-VLT spectra of a sample of four damped Ly $\alpha$ systems (DLAs) toward the quasars Q0100+13, Q1331+17, Q2231-00 and Q2343+12 with the existing HIRES-Keck spectra, we covered the total optical spectral range from 3150 to $10000 \AA$ for the four quasars. This large wavelength coverage and the high quality of the spectra allowed us to measure the column densities of up to 21 ions, namely of 15 elements - N, O, Mg, Al, Si, P, S, Cl, Ar, Ti, Cr, Mn, Fe, Ni, Zn. This comprehensive set of ionic column densities and elemental abundances severely contrasts with the majority of DLAs for which only a handful of ions and elements is typically observed. Such a large amount of information is necessary to constrain the photoionization and dust depletion effects, two important steps in order to derive the intrinsic chemical abundance patterns of DLAs. We evaluated the photoionization effects with the help of the $\mathrm{Al}^{+} / \mathrm{Al}^{++}, \mathrm{Fe}^{+} / \mathrm{Fe}^{++}, \mathrm{N}^{0} / \mathrm{N}^{+}$and $\mathrm{Ar} / \mathrm{Si}, \mathrm{S}$ ratios, and computed dust corrections. Our analysis revealed that the DLA toward Q2343+12 requires important ionization corrections. This makes the abundance determinations in this object uncertain. The access to the complete series of relatively robust intrinsic elemental abundances in the other three DLAs allowed us to constrain their star formation history, their age and their star formation rate by a detailed comparison with a grid of chemical evolution models for spiral and dwarf irregular galaxies. Our results show that the galaxies associated with these three DLAs in the redshift interval $z_{\mathrm{abs}}=1.7-2.5$ are either outer regions of spiral disks (radius $\geq 8 \mathrm{kpc}$ ) or dwarf irregular galaxies (showing a bursting or continuous star formation history) with ages varying from some $50 \mathrm{Myr}$ only to $\gtrsim 3.5 \mathrm{Gyr}$ and with moderate star formation rates per unit area of $-2.1<\log \psi<-1.5 M_{\odot} \mathrm{yr}^{-1} \mathrm{kpc}^{-2}$.
\end{abstract}

Key words. cosmology: observations - galaxies: abundances - galaxies: evolution - galaxies: quasars: absorption lines

\section{Introduction}

The study of high redshift galaxies and the access to their physical properties can be done using either emission or absorption line spectroscopy. Absorption line spectroscopy is a very powerful technique, and it presents several advantages compared to the information which can be derived from emission. First, it allows one to detect objects up to very high redshift. Indeed, the quasars (QSOs) detected up to redshifts of 6.2 (Fan et al. 2001) can be used as background searchlights for useful probes of the intervening Universe up to lookback times of $95 \%$ of the age of the Universe. Second, through the analysis of QSO absorption lines we can study the spatial distribution, motion, chemical enrichment and ionization histories of gaseous structures on a variety of scales, ranging from the intergalactic medium to high

Send offprint requests to: M. Dessauges-Zavadsky, e-mail: miroslava.dessauges@obs.unige.ch

* Based on the UVES observations collected during the ESO programmes ID No. 65.O-0296 and 67.A-0022 at the VLT/Kueyen telescope, Paranal, Chile. column density absorption systems associated with galaxies. Third, since the detection of material intercepting a line of sight to a given QSO is dependent only on the column density of the gas and the luminosity of the QSO, this is a unique technique for probing the chemical composition and physical conditions in the interstellar medium of various types of galaxies over a large range of lookback times, detected independently of their distance, luminosity, star formation history and morphology.

While the major part of the baryon content of the presentday galaxies is concentrated in stars, an epoch must have existed at which the essential of the mass of galaxies still lay in the gas. The damped Ly $\alpha$ systems (DLAs) observed in QSO spectra are characterized by large column densities of neutral hydrogen, $>2 \times 10^{20} \mathrm{~cm}^{-2}$, and dominate the cosmic mass density of neutral hydrogen gas (e.g. Storrie-Lombardi et al. 1996; Storrie-Lombardi \& Wolfe 2000). The general view is that the DLA systems probably represent some early stages in the evolution of the galaxies we see around us today, perhaps at a time shortly after they had condensed out of the intergalactic medium, but before they had time to form many stars, 
so that most of their mass still resided in the interstellar medium. These objects thus are by far our best laboratory for studying the galaxies at high redshift, in their early stages of evolution, and for tracking the galactic chemical evolution through the cosmic ages.

However, the reconstruction of the star formation histories of DLAs from the abundance pattern measurements is not straightforward and led to contradictory results (Lu et al. 1996; Prochaska \& Wolfe 1999; Centurión et al. 2000; Molaro et al. 2000; Vladilo 2002a). The access to abundance ratios involving two elements formed on different timescales, in particular the $\alpha / \mathrm{Fe}$ ratios, examined together with $[\mathrm{Fe} / \mathrm{H}]$, or any other metallicity tracer such as $[\mathrm{Zn} / \mathrm{H}]$, is crucial, since the star formation history of a galaxy is completely determined by the $[\alpha / \mathrm{Fe}]$ versus $[\mathrm{Fe} / \mathrm{H}]$ distributions (e.g. Matteucci 2001). The principal difficulty, however, is to disentangle the nucleosynthetic contributions from dust depletion effects. Because we are studying gas-phase elemental abundances in DLAs, the observed abundances may not represent the intrinsic composition of the system if part of the elements is removed from the gas to the solid phase (Savage \& Sembach 1996). Several pieces of evidence show that some dust is indeed present in DLAs (e.g. Pei et al. 1991; Prochaska \& Wolfe 2002). Another effect which has to be carefully examined, although usually assumed negligible is the photoionization effect (e.g. Viegas 1995; Vladilo et al. 2001), since in gas-phase studies a fraction of the gas may also be ionized.

The difficulty to correctly evaluate the photoionization effects and the degeneracy between dust depletion and nucleosynthesis in DLAs is accentuated by the limited number of ions and elements typically detected in these galaxies. To determine the "intrinsic" chemical abundance patterns, free from ionization and dust depletion effects and to be then able to study the DLA galaxies individually, we need to examine several column density ratios of adjacent ions of the same element and the relative abundances of as many elements as possible. We thus aimed at obtaining for a few damped Ly $\alpha$ systems the column density measurements of many ions of different ionization levels and the abundance measurements of the complete series of accessible elements in DLAs. Until now the DLA galaxy population has been analyzed as a whole and chemical evolution models were constructed in order to interpret the abundance patterns observed in DLAs as an ensemble, considering them as an evolutionary sequence (e.g. Matteucci et al. 1997; Jimenez et al. 1999; Hou et al. 2001; Mathlin et al. 2001), while several pieces of evidence - the low redshift deep imaging revealing a variety of morphological types belonging to the DLA population (e.g. Le Brun et al. 1997; Nestor et al. 2002), the large scatter in the $\alpha$ over Fe-peak element abundance ratios at a given metallicity and the large scatter observed in the metallicities show that the DLAs trace galaxies with different evolutionary histories. Some may have formed stars on timescales similar to that of the early Milky Way, while others apparently did so more slowly or intermittently, so that the Fe-peak elements could catch up with the $\alpha$-elements. The DLA systems thus likely sample a wide range of galaxy types, and consequently a variety of star formation histories. It is very important to determine the star formation history of each of these high redshift galaxies individually to better understand the galaxy formation and evolution.

We thus attempted a new approach for studying the DLA galaxy population, focusing on individual systems. By combining our UVES-VLT spectra with the existing HIRES-Keck spectra we obtained the column density measurements of some 21 ions, namely of 15 elements - N, O, Mg, Al, Si, P, S, Cl, Ar, $\mathrm{Ti}, \mathrm{Cr}, \mathrm{Mn}, \mathrm{Fe}, \mathrm{Ni}, \mathrm{Zn}-$, in four DLA systems in the redshift interval $z_{\mathrm{abs}}=1.7-2.5$. The complete analysis of the four DLA systems is described in Sects. 2 and 3. In Sect. 4, we address the question of photoionization effects, and in Sect. 5 we analyze the dust content and discuss for each system individually the intrinsic chemical abundances. Finally, the relative abundances as a function of the metallicity and the redshift are systematically compared with a grid of chemical evolution models for spiral and dwarf irregular galaxies in Sect. 6.

\section{Observations and data reduction}

The selected quasars Q0100+13, Q1331+17, Q2231-00 and Q2343+12 with four intervening DLAs in the redshift interval $z_{\text {abs }}=1.7-2.5$ are relatively bright with $V=16.5-17.5$ and already known from the literature. Indeed, the DLAs toward Q0100+13, Q1331+17 and Q2231-00 were carefully and accurately analyzed by Prochaska \& Wolfe (1999) and Prochaska et al. (2001) thanks to high-resolution spectra obtained with the HIRES echelle spectrograph on the Keck I telescope at Mauna Kea, in Hawaii, and some results also obtained from HIRESKeck spectra on the DLA toward Q2343+12 were presented by e.g. Lu et al. (1998).

To complete the wavelength coverage of the HIRES-Keck spectra of these quasars, we used the unique capability of the Ultraviolet-Visual Echelle Spectrograph UVES (D'Odorico et al. 2000) on the VLT $8.2 \mathrm{~m}$ Kueyen ESO telescope at Paranal, Chile, and we obtained high resolution, high signal-tonoise ratio spectra for each quasar in the blue $\lambda=3150-4500 \AA$ and in the far-red $\lambda=6700-10000 \AA$. The observations were performed in visitor mode in September 2000 for one object and in service mode in summer 2001 for the three other objects under good seeing conditions (between $0.5^{\prime \prime}$ and $1.0^{\prime \prime}$ ). For each observation, slit widths of $1^{\prime \prime}$ in the blue and of $0.9^{\prime \prime}$ in the red were chosen with a CCD binning of $2 \times 2$ resulting in a resolution of $F W H M \simeq 6.9 \mathrm{~km} \mathrm{~s}^{-1}$ and $6.4 \mathrm{~km} \mathrm{~s}^{-1}$, respectively. Relevant details of the observations are collected in Table 1. The total exposure times of each quasar were split in multiple exposures of 3600 or $4500 \mathrm{~s}$.

The spectra were reduced using the ESO data reduction package MIDAS and the UVES pipeline in an interactive mode available as a MIDAS context. A detailed description of the pipeline can be found in Ballester et al. (2000). To make sure of the best result, we made a systematic check of each step of the pipeline reduction. The wavelengths of the reduced onedimensional spectra were converted to a vacuum-heliocentric scale, and the individual spectra of each object were co-added using their signal-to-noise ratio as weights in order to get the maximum signal-to-noise ratios. The final step was the normalization of the resulting spectra obtained by dividing them by a spline function fitted to smoothly connect the regions 
Table 1. Journal of observations.

\begin{tabular}{lccclccc}
\hline \hline Quasar & $\begin{array}{c}V \\
{[\mathrm{mag}]}\end{array}$ & $z_{\mathrm{em}}$ & $z_{\mathrm{abs}}$ & Observing date & Mode & $\begin{array}{c}\text { Wavelength range } \\
{[\mathrm{nm}]}\end{array}$ & $\begin{array}{c}\text { Exposure time } \\
{[\mathrm{s}]}\end{array}$ \\
\hline Q0100+13 & 16.6 & 2.69 & 2.309 & September 2001 & dic2 $(390+860)$ & $330-452 / 670-1000$ & 7200 \\
Q1331+17 & 16.7 & 2.08 & 1.776 & June-July 2001 & $\operatorname{dic} 1(346+860)$ & $312-387 / 670-1000$ & 13500 \\
Q2231-00 & 17.5 & 3.02 & 2.066 & September 2000 & $\operatorname{dic} 2(390+800)$ & $335-452 / 610-990$ & 15700 \\
Q2343+12 & 17.0 & 2.55 & 2.431 & August 2001 & $\operatorname{dic} 1(346+860)$ & $315-387 / 670-1000$ & 14400 \\
\hline
\end{tabular}

free from absorption features. The continuum in the Ly $\alpha$ forest was fitted by using small regions deemed to be free of absorptions and by interpolating between these regions with a spline. An average signal-to-noise ratio per pixel of $\sim 30,55$ and 45 was achieved in the final spectra at $\lambda \sim 3700 \AA, 7500 \AA$ and $9000 \AA$ A, respectively.

\section{Data analysis and ionic column densities}

By combining our UVES-VLT spectra with the existing HIRES-Keck spectra obtained by Prochaska \& Wolfe (1999) we cover the total spectral range from 3150 to $10000 \AA$ for the four observed quasars, and hence have access for the first time to up to 21 ions and 15 elements for each of their intervening DLAs.

The column densities of the metal species were derived with the Voigt profile fitting technique. This technique consists in fitting theoretical Voigt profiles to the observed DLA absorption metal lines well described as a complex of components, each defined by a redshift $z$, a Doppler parameter $b$, a column density $N$ and the corresponding errors. The fits were performed using a $\chi^{2}$ minimization routine fitlyman in MIDAS (Fontana \& Ballester 1995). We assumed that metal species with similar ionization potentials can be fitted using identical component fitting parameters, i.e. the same $b$ (which means that macroturbulent motions dominate over thermal broadening) and the same $z$ in the same component, and allowing for variations from metal species to metal species in $N$ only. We distinguish three categories of metal species with similar ionization potentials: the low-ion transitions (i.e. the neutral and singly ionized species), the intermediate-ion transitions (e.g. Fe III, Al III), and the high-ion transitions (e.g. C IV, Si IV). We used relatively strong (but not saturated) lines to fix the component fitting parameters ( $b$ and $z$ ), and excellent profile fits could then be achieved for weak metal lines and for metal lines located in the Ly $\alpha$ forest where the probability of blending is high by allowing only the column density to vary. We had a sufficient number of relatively strong metal-line profiles to well constrain the fitting parameters in the four studied DLAs exhibiting multicomponent velocity structures.

Throughout the analysis we adopted the list of atomic data - laboratory wavelengths and oscillator strengths - presented in Table 2. The most recent measurements of $\lambda$ and $f$-values of the metal-ions that impact the abundances of DLA systems and their references are reported there. Information on the atomic data of additional useful metal-ions can be found in Prochaska et al. (2001) and on the web site of "The HIRES Damped Ly $\alpha$
Abundance Database" ${ }^{\text {1 }}$ maintained by Prochaska and collaborators. Finally, we adopt the solar meteoritic abundances from Grevesse \& Sauval (1998).

The measured component per component ionic column densities obtained from the fitting model solutions of the lowand intermediate-ion transitions are summarized in Tables 3-7. The reported errors are the $1 \sigma$ errors on the fits computed by fitlyman. They possibly underestimate the real error on the measure, since they do not take into account the uncertainty on the continuum level determination. For the components where the line profile is saturated, the column densities are listed as lower limits. The values reported as upper limits are cases with significant line blending with $\mathrm{H}$ I clouds of the Ly $\alpha$ forest or with telluric lines. The fitting solutions of the low- and intermediate-ion transitions are shown in Figs. 2, 4, 6 and 8 for the four DLA. In these velocity plots, $v=0$ corresponds to an arbitrary component, and all the identified components are marked by small vertical bars. The thin solid line represent the best-fitting solution. The telluric lines have been identified thanks to the spectra of a hot, fast rotating star taken in the same nights as the scientific exposures.

The neutral hydrogen column densities were estimated from the fits of the Ly $\alpha$ damping line profiles. The $b$-values were fixed at $20 \mathrm{~km} \mathrm{~s}^{-1}$, and the redshift $z$ were left as a free parameter or fixed to the redshift of the strongest component of the metal-line profiles according to the system (see comments in the following sub-Sections). When other lines of the Lyman series were accessible in our spectra, we used them to check the H I column densities derived from the Ly $\alpha$ lines. Figures 1, 3, 5 and 7 show the results of the H I fitting solutions for the four DLA systems. The small vertical bar corresponds to the redshift obtained in the best-fitting solution and the thin solid line represents the best fit.

We now briefly describe the fitting results for the four individual DLA systems. Their Mn II $\lambda 2576,2594,2606$, Ti II $\lambda_{\text {rest }}>3000$ and Mg II $\lambda 1239,1240$ fits and column density measurements have already been discussed in details in Dessauges-Zavadsky et al. (2002a, hereafter Paper I). Let's just remind that the $\mathrm{Mg}$ II $\lambda 1239,1240$ lines are detected in the DLA Ly $\alpha$ damping line red wing and that they have been fitted after a local renormalization of the spectrum around the $\mathrm{Mg}$ II lines with the fit of the Ly $\alpha$ damping wing profile. The errors on the measured $\mathrm{Mg}^{+}$column densities have been estimated by varying the continuum level by $5 \%$.

\footnotetext{
${ }^{1}$ http://kingpin.ucsd.edu/ hiresdla/
} 
Table 2. Atomic data.

\begin{tabular}{|c|c|c|c|c|c|c|c|}
\hline Transition & $\lambda$ & $f$-value & Reference & Transition & $\lambda$ & $f$-value & Reference \\
\hline О I $\lambda 950$ & 950.8846 & 0.00157100 & 1 & С I $\lambda 1328$ & 1328.8333 & 0.05804000 & 1 \\
\hline Р II $\lambda 961$ & 961.0410 & 0.34890000 & 1 & С II $\lambda 1334$ & 1334.5323 & 0.12780000 & 1 \\
\hline Р ІІ $\lambda 963$ & 963.8010 & 1.45800000 & 1 & C II ${ }^{*} \lambda 1335$ & 1335.7077 & 0.11490000 & 1 \\
\hline $\mathrm{N}$ I $\lambda 963.9$ & 963.9903 & 0.01837000 & 1 & $\mathrm{Cl}$ I $\lambda 1347$ & 1347.2400 & 0.11860000 & 1 \\
\hline N I $\lambda 964.6$ & 964.6256 & 0.01180000 & 1 & О $\lambda \lambda 1355$ & 1355.5977 & 0.00000124 & 1 \\
\hline N I $\lambda 965.0$ & 965.0413 & 0.00580100 & 1 & Ni II $\lambda 1370$ & 1370.1310 & 0.07690000 & 5 \\
\hline О г $\lambda 971$ & 971.7380 & 0.01480000 & 1 & Si II $\lambda 1526$ & 1526.7066 & 0.12700000 & 6 \\
\hline О $\lambda 2976$ & 976.4481 & 0.00330000 & 1 & С $л \lambda 1560$ & 1560.3092 & 0.08041000 & 1 \\
\hline C III $\lambda 977$ & 977.0200 & 0.76200000 & 1 & Fe II $\lambda 1608$ & 1608.4511 & 0.05800000 & 7 \\
\hline Ог $\lambda 988$ & 988.7734 & 0.04318000 & 1 & Fe II $\lambda 1611$ & 1611.2005 & 0.00136000 & 2 \\
\hline Si II $\lambda 989$ & 989.8731 & 0.13300000 & 1 & С $\lambda 1656$ & 1656.9283 & 0.14050000 & 1 \\
\hline $\mathrm{Cl} \lambda \lambda 1002$ & 1002.3464 & 0.07046000 & 1 & Al II $\lambda 1670$ & 1670.7874 & 1.88000000 & 1 \\
\hline S III $\lambda 1012$ & 1012.5020 & 0.03550000 & 1 & Ni II $\lambda 1709$ & 1709.6000 & 0.03240000 & 8 \\
\hline Si II $\lambda 1020$ & 1020.6989 & 0.02828000 & 1 & Ni II $\lambda 1741$ & 1741.5490 & 0.04270000 & 8 \\
\hline $\operatorname{Ly} \beta$ & 1025.7223 & 0.07912000 & 1 & Ni II $\lambda 1751$ & 1751.9100 & 0.02770000 & 8 \\
\hline $\mathrm{Cl}$ I $\lambda 1028$ & 1028.1740 & 0.02228000 & 1 & Si II $\lambda 1808$ & 1808.0126 & 0.00218000 & 9 \\
\hline $\mathrm{Cl} \mathrm{I} \lambda 1030$ & 1030.8845 & 0.02870000 & 1 & $\mathrm{Al}$ III $\lambda 1854$ & 1854.7164 & 0.53900000 & 1 \\
\hline $\mathrm{Cl} \mathrm{I} \lambda 1031$ & 1031.5070 & 0.15060000 & 1 & Al III $\lambda 1862$ & 1862.7895 & 0.26800000 & 1 \\
\hline С ІІ $\lambda 1036$ & 1036.3367 & 0.12310000 & 1 & Ті ІІ $\lambda 1910.60$ & 1910.6000 & 0.20200000 & 10 \\
\hline О г $\lambda 1039$ & 1039.2304 & 0.00919700 & 1 & Ті II $\lambda 1910.97$ & 1910.9700 & 0.09800000 & 10 \\
\hline $\operatorname{Ar} \mathrm{I} \lambda 1048$ & 1048.2199 & 0.24410000 & 1 & Zn II $\lambda 2026$ & 2026.1360 & 0.48900000 & 11 \\
\hline $\operatorname{Ar} I \lambda 1066$ & 1066.6600 & 0.06652000 & 1 & Cr II $\lambda 2026$ & 2026.2690 & 0.00471000 & 12 \\
\hline N II $\lambda 1083$ & 1083.9900 & 0.10310000 & 1 & Mg I $\lambda 2026$ & 2026.4768 & 0.11200000 & 1 \\
\hline Fe III $\lambda 1122$ & 1122.5260 & 0.05390000 & 2 & Cr II $\lambda 2056$ & 2056.2539 & 0.10500000 & 11 \\
\hline $\mathrm{N} I \lambda 1134.165$ & 1134.1653 & 0.01342000 & 1 & CrII $\lambda 2062$ & 2062.2340 & 0.07800000 & 11 \\
\hline N I $\lambda 1134.415$ & 1134.4149 & 0.02683000 & 1 & Zn II $\lambda 2062$ & 2062.6640 & 0.25600000 & 11 \\
\hline $\mathrm{N}$ I $\lambda 1134.98$ & 1134.9803 & 0.04023000 & 1 & Cr II $\lambda 2066$ & 2066.1610 & 0.05150000 & 11 \\
\hline P IIג1152 & 1152.8180 & 0.23600000 & 1 & Fe II $\lambda 2249$ & 2249.8768 & 0.00182100 & 13 \\
\hline С $2 \lambda 1157$ & 1157.1857 & 0.02440000 & 1 & Fe II $\lambda 2260$ & 2260.7805 & 0.00244000 & 13 \\
\hline S III $\lambda 1190$ & 1190.2080 & 0.02217000 & 1 & Fe II $\lambda 2344$ & 2344.2140 & 0.11400000 & 14 \\
\hline N I $\lambda 1199.55$ & 1199.5496 & 0.13280000 & 1 & Fe II $\lambda 2374$ & 2374.4612 & 0.03130000 & 14 \\
\hline N I $\lambda 1200.22$ & 1200.2233 & 0.08849000 & 1 & Fe II $\lambda 2382$ & 2382.7650 & 0.32000000 & 14 \\
\hline N I $\lambda 1200.71$ & 1200.7098 & 0.04423000 & 1 & Mn II $\lambda 2576$ & 2576.8770 & 0.35080000 & 1 \\
\hline Si III $\lambda 1206$ & 1206.5000 & 1.66000000 & 1 & Fe II $\lambda 2586$ & 2586.6500 & 0.06910000 & 14 \\
\hline $\operatorname{Ly} \alpha$ & 1215.6701 & 0.41640000 & 1 & Mn II $\lambda 2594$ & 2594.4990 & 0.27100000 & 1 \\
\hline Mg II $\lambda 1239$ & 1239.9250 & 0.00063000 & 3 & Fe II $\lambda 2600$ & 2600.1729 & 0.23900000 & 14 \\
\hline Mg II $\lambda 1240$ & 1240.3950 & 0.00035000 & 3 & Mn II $\lambda 2606$ & 2606.4620 & 0.19270000 & 1 \\
\hline S II $\lambda 1250$ & 1250.5840 & 0.00545300 & 1 & Mg II $\lambda 2796$ & 2796.3520 & 0.61230000 & 15 \\
\hline S II $\lambda 1253$ & 1253.8110 & 0.01088000 & 1 & Mg II $\lambda 2803$ & 2803.5310 & 0.30540000 & 15 \\
\hline S II $\lambda 1259$ & 1259.5190 & 0.01624000 & 1 & $\operatorname{Mg} \mathrm{I} \lambda 2852$ & 2852.9642 & 1.81000000 & 1 \\
\hline С I $\lambda 1277$ & 1277.2450 & 0.09665000 & 1 & Ti II $\lambda 3067$ & 3067.2451 & 0.04655000 & 1 \\
\hline С $2 \lambda 1280$ & 1280.1353 & 0.02432000 & 1 & Ti II $\lambda 3073$ & 3073.8770 & 0.10910000 & 1 \\
\hline Тi III $\lambda 1298$ & 1298.6970 & 0.09507000 & 1 & Ti II $\lambda 3230$ & 3230.1310 & 0.05860000 & 1 \\
\hline Огд1302 & 1302.1685 & 0.04887000 & 1 & Тi II $\lambda 3242$ & 3242.9290 & 0.18320000 & 1 \\
\hline Si II $\lambda 1304$ & 1304.3702 & 0.09400000 & 4 & Ті II $\lambda 3384$ & 3384.7400 & 0.34000000 & 1 \\
\hline Ni II $\lambda 1317$ & 1317.2170 & 0.07786000 & 5 & & & & \\
\hline
\end{tabular}

References: (1) Morton (1991), (2) Raassen \& Uylings (1998), (3) Theodosiou \& Federman (1999), (4) Tripp et al. (1996), (5) Fedchak \& Lawler (1999), (6) Schectman et al. (1998), (7) Bergeson et al. (1996), (8) Fedchak et al. (2000), (9) Bergeson \& Lawler (1993a), (10) Wiese et al. (2001), (11) Bergeson \& Lawler (1993b), (12) Verner et al. (1994), (13) Bergeson et al. (1994), (14) Morton (2000, 2001), (15) Verner (1996).

\section{1. $Q 0100+13, z_{a b s}=2.309$}

This system was carefully studied by Wolfe et al. (1994), and subsequently by Prochaska \& Wolfe (1999) and Prochaska et al. (2001). Thanks to the UVES spectra we confirm some of their column density measurements, namely $N\left(\mathrm{Zn}^{+}\right), N\left(\mathrm{Cr}^{+}\right)$ and $N\left(\mathrm{Fe}^{+}\right)$obtained from the Fe II $\lambda 2249,2260,2344$, 2374 lines not present in the HIRES spectra, and we obtain new column density measurements of S II, Ar I, P II, N I and Mg II (see Fig. 2). We also get upper limits on the column densities of two intermediate-ions, N II and Fe III located in the Ly $\alpha$ forest, by using the profile shapes of the intermediate-ion lines $\mathrm{Al}$ III $\lambda 1854,1862$ observed in the HIRES spectra to constrain the fitting parameters (see Fig. 2). 
Table 3. Component structure of the $z_{\mathrm{abs}}=2.309$ DLA system toward Q0100+13.

\begin{tabular}{|c|c|c|c|c|c|}
\hline No. & $z_{\mathrm{abs}}$ & $\begin{array}{c}v_{\text {rel }}^{*} \\
\mathrm{~km} \mathrm{~s}^{-1}\end{array}$ & $\begin{array}{c}b\left(\sigma_{b}\right) \\
\mathrm{km} \mathrm{s}^{-1}\end{array}$ & Ion & $\log N\left(\sigma_{\log N}\right)$ \\
\hline \multicolumn{6}{|c|}{ Low-ion transitions } \\
\hline \multirow[t]{8}{*}{1} & 2.309027 & 0 & $5.6(0.1)$ & Fe II & $14.86(0.01)$ \\
\hline & & & & $\mathrm{Zn}$ II & $12.28(0.01)$ \\
\hline & & & & Cr II & $13.15(0.01)$ \\
\hline & & & & S II & $14.85(0.05)$ \\
\hline & & & & Ar I & $13.99(0.15)$ \\
\hline & & & & P II & $12.88(0.08)$ \\
\hline & & & & $\mathrm{N}_{\mathrm{I}}$ & $14.79(0.08)$ \\
\hline & & & & Mg II & $15.41(0.07)$ \\
\hline \multirow[t]{8}{*}{2} & 2.309161 & +12 & $5.3(0.1)$ & $\mathrm{Fe} I I$ & $14.61(0.01)$ \\
\hline & & & & $\mathrm{Zn}$ II & $12.03(0.02)$ \\
\hline & & & & Cr II & $12.96(0.01)$ \\
\hline & & & & S II & $14.73(0.08)$ \\
\hline & & & & Ar I & $13.81(0.08)$ \\
\hline & & & & P II & $12.55(0.10)$ \\
\hline & & & & N I & $14.65(0.12)$ \\
\hline & & & & Mg II & $15.06(0.13)$ \\
\hline 3 & 2.309443 & +37 & $14.1(0.2)$ & $\mathrm{Fe}$ II & $14.02(0.03)$ \\
\hline 4 & 2.309486 & +52 & $2.8(0.3)$ & Fe II & $12.96(0.03)$ \\
\hline \multicolumn{6}{|c|}{ Intermediate-ion transitions } \\
\hline \multirow[t]{3}{*}{1} & 2.308980 & -4 & $6.0(1.9)$ & Al III & $12.18(0.12)$ \\
\hline & & & & Fe III & $<13.28$ \\
\hline & & & & $\mathrm{N}$ II & $<13.24$ \\
\hline \multirow[t]{3}{*}{2} & 2.309093 & +6 & $11.2(1.2)$ & Al III & $12.22(0.08)$ \\
\hline & & & & $\mathrm{Fe}$ III & $<13.22$ \\
\hline & & & & $\mathrm{N}$ II & $<13.64$ \\
\hline 3 & 2.309498 & +43 & $13.0(1.9)$ & Al III & $12.12(0.05)$ \\
\hline
\end{tabular}

* Velocity relative to $z=2.309027$.

The low-ion absorption line profiles of this system are characterized by 4 components. Their redshifts, $b$-values and column densities are presented in Table 3. Two of the 4 components, the components 3 and 4, are too weak to be accurately identified in the weak metal-lines and in the metal-lines located in the Ly $\alpha$ forest. Thus, we prefer to avoid measuring them in most of the observed transitions. Being weak and since we are interested in the relative elemental abundances, this has no impact on the final conclusions on this DLA system. The intermediate-ion lines show very similar profiles to the low-ion profiles suggesting they are coming from the same absorption regions. Their fitting solutions are given in Table 3 .

Figure 1 shows the fitting solutions of two Lyman lines of this DLA, Ly $\alpha$ and $\operatorname{Ly} \beta$, observed in the UVES spectra. The fits were obtained by fixing the $b$-value at $20 \mathrm{~km} \mathrm{~s}^{-1}$ and the redshift at $z=2.309027$, i.e. at the redshift of the strongest metalline component (the component 1 ). The measured H I column density, $\log N(\mathrm{HI})=21.37 \pm 0.08$, is very similar to the one derived by Pettini et al. (1990) from low-resolution spectra. Giving the high $\mathrm{H}^{0}$ column density, the red wing of the Ly $\alpha$ damping profile extends over the S II $\lambda 1250,1253,1259$ lines. Similarly to the Mg II $\lambda 1240$ line (the Mg II $\lambda 1239$ line being heavily blended), we had first to locally renormalize the spectrum with the fit of the Ly $\alpha$ damping wing profile before measuring the $\mathrm{S}^{+}$column density.
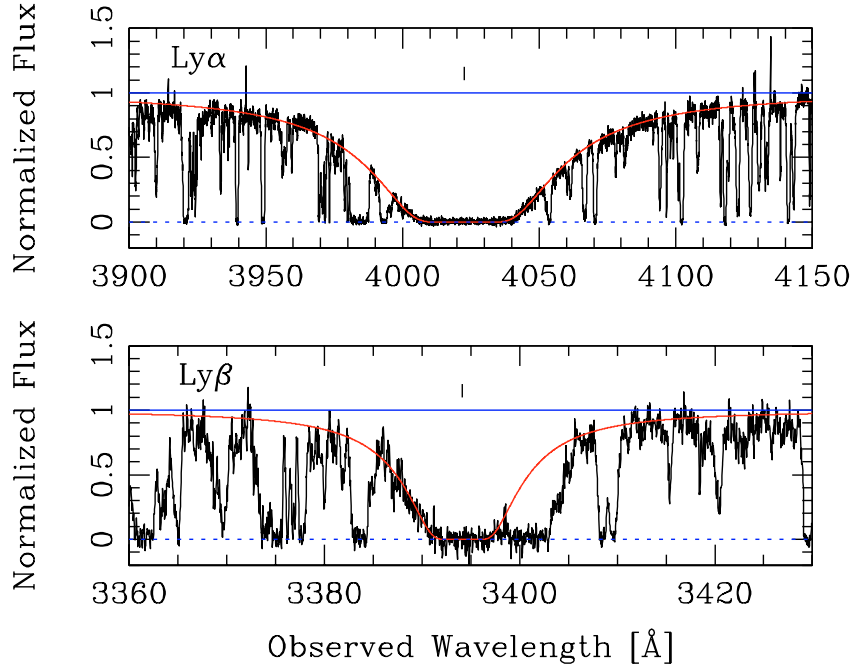

Fig. 1. Normalized UVES spectra of Q0100+13 showing the DLA Ly $\alpha$ and $\operatorname{Ly} \beta$ profiles with the Voigt profile fits. The vertical bar corresponds to the wavelength centroid of the component used for the best fit, $z=2.309027$. The measured HI column density is $\log N(\mathrm{H} i)=21.37 \pm 0.08$.

\section{2. $Q 1331+17, Z_{a b s}=1.776$}

This famous DLA system has been studied by a large number of authors. We mention here only the work of Prochaska \& Wolfe (1999) and Prochaska et al. (2001) who obtained the highest quality data and made the most accurate analysis. By extending their wavelength coverage with our UVES data, we obtained additional column density measurements of S II, P II, N I, Mg II, Mn II and Cli. We confirm the Prochaska \& Wolfe (1999) and Prochaska et al. (2001) column density measurements of Si II, Fe II, C I, Mg I and Al III (see Fig. 4). But, their $N\left(\mathrm{Ni}^{+}\right)$and $N\left(\mathrm{Cr}^{+}\right)$measurements differ from ours by almost 0.1 dex. We also confirm the revised value of $N\left(\mathrm{Zn}^{+}\right)$of Prochaska et al. (2001) obtained by correcting the contamination of Zn II $\lambda 2026$ by the Mg I $\lambda 2026$ profile. The detection of the relatively strong $\mathrm{Mg}$ I $\lambda 2852$ line in the UVES spectra has allowed us to measure even more reliably this contamination. Finally, we obtain a more constraining upper limit for $N\left(\mathrm{Ti}^{+}\right)$from the Ti II $\lambda 3384$ line than the higher value deduced by Prochaska et al. (2001) from the Ti II $\lambda 1910$ lines having a 3 times lower oscillator strength.

The fitting solutions of the detected components in the lowion metal-lines are presented in Table 4. A surprising particularity of this system known as a system exhibiting the largest dust depletion level of any DLA is that the fits of the refractory element lines require 6 components ( 2 components at $v \sim 0 \mathrm{~km} \mathrm{~s}^{-1}$, see Fig. 4), while the non-refractory and mildly refractory element lines are well fitted with 5 components only ( 1 component at $v=0 \mathrm{~km} \mathrm{~s}^{-1}$, see Fig. 4). The refractory elements showing weak absorption lines, like the Mg II $\lambda 1239,1240$ and Cr II $\lambda 2056,2062$ lines, are borderline cases, in the sense that the component 1 is detected but only marginally. Concerning the component 6 , it is only observed in the strongest metal-line transitions.

The detection of the $\mathrm{Cl}$ I $\lambda 1347$ line is exceptional. $\mathrm{Cl}^{0}$ has previously been observed only by Ledoux et al. (2002) and 

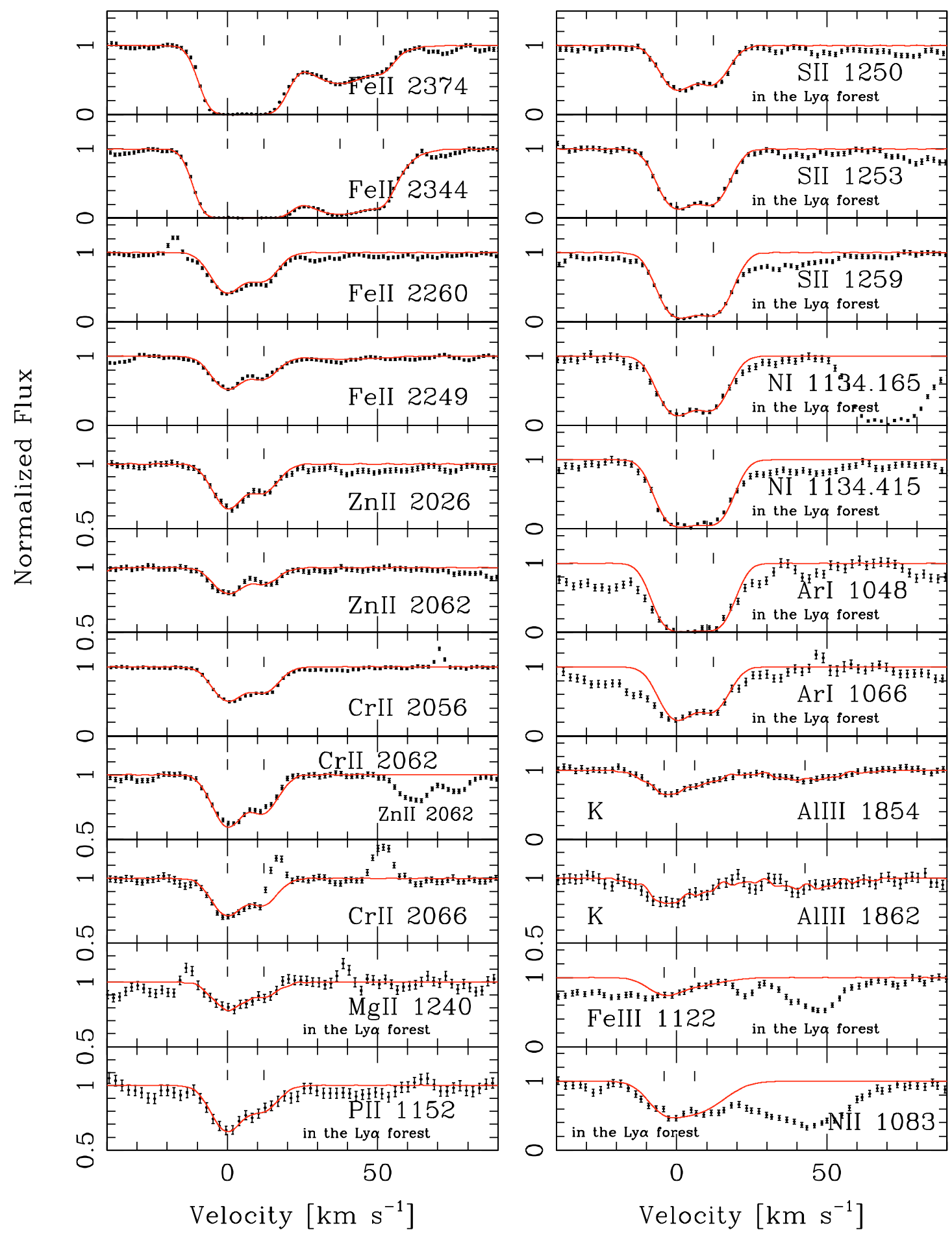

Fig. 2. Velocity plots of the metal line transitions (normalized intensities shown by dots with $1 \sigma$ error bars) for the DLA toward Q0100+13. The zero velocity is fixed at $z=2.309027$. For this and all the following figures with velocity plots, the vertical bars mark the positions of the fitted velocity components and the symbols $\oplus$ correspond to the telluric lines. The letter K refers to the Keck spectra.

Prochaska et al. (2003) in the DLA toward Q0551-366 and in the DLA toward FJ081240.6+320808, respectively. In the DLA system studied in this paper, we detect only the components 2 and 3 of the $\mathrm{Cl}$ I $\lambda 1347$ line, the other components 4, 5 and 6 are blended with $\operatorname{Ly} \alpha$ forest absorptions. However, since the line profiles of S II $\lambda 1250$, Si II $\lambda 1808$ and C I $\lambda 1656$ show very little absorption at $v>25 \mathrm{~km} \mathrm{~s}^{-1}$ (only $\sim 20 \%$ of the total column density for S II and Si II), we consider the $\mathrm{Cl}$ I column density derived from the components 2 and 3 as a value. We adopt an error on $N(\mathrm{Cl}$ I) of 0.10 dex to account for the possible contribution from the blended components 4,5 and 6. Nevertheless, the $\mathrm{Cl}^{0}$ column density measurement provides only a strict lower limit on the $\mathrm{Cl}$ absolute abundance of $[\mathrm{Cl} / \mathrm{H}]>-1.37$, since the dominant state of $\mathrm{Cl}$ should be $\mathrm{Cl}$ II 
Table 4. Component structure of the $z_{\mathrm{abs}}=1.776$ DLA system toward Q1331+17.

\begin{tabular}{|c|c|c|c|c|c|c|c|c|c|c|c|}
\hline No. & $z_{\mathrm{abs}}$ & $\begin{array}{c}v_{\text {rel }}^{*} \\
\mathrm{~km} \mathrm{~s}^{-1}\end{array}$ & $\begin{array}{c}b\left(\sigma_{b}\right) \\
\mathrm{km} \mathrm{s}^{-1}\end{array}$ & Ion & $\log N\left(\sigma_{\log N}\right)$ & No. & $z_{\mathrm{abs}}$ & $\begin{array}{c}v_{\text {rel }}^{*} \\
\mathrm{~km} \mathrm{~s}^{-1}\end{array}$ & $\begin{array}{c}b\left(\sigma_{b}\right) \\
\mathrm{km} \mathrm{s}^{-1}\end{array}$ & Ion & $\log N\left(\sigma_{\log N}\right)$ \\
\hline \multicolumn{12}{|c|}{ Low-ion transitions } \\
\hline \multirow[t]{5}{*}{1} & 1.776336 & -4 & $12.0(0.3)$ & Fe II & $13.90(0.04)$ & 4 & 1.776724 & +38 & $13.4(0.8)$ & Fe II & $14.07(0.02)$ \\
\hline & & & & Cr II & $9.00(0.25)$ & & & & & $\mathrm{Zn} \mathrm{II}$ & $11.50(0.06)$ \\
\hline & & & & Ni II & $12.82(0.13)$ & & & & & Cr II & $12.22(0.06)$ \\
\hline & & & & Mn II & $11.84(0.06)$ & & & & & Ni II & $12.87(0.04)$ \\
\hline & & & & Mg II & $11.00(0.25)$ & & & & & Mn II & $11.91(0.01)$ \\
\hline \multirow[t]{12}{*}{2} & 1.776370 & 0 & $9.8(0.1)$ & $\mathrm{Fe}$ II & $14.16(0.02)$ & & & & & Si II & $14.60(0.01)$ \\
\hline & & & & Zn II & $12.35(0.01)$ & & & & & S II & $14.15(0.11)$ \\
\hline & & & & Cr II & $12.67(0.01)$ & & & & & P II & $12.41(0.21)$ \\
\hline & & & & Ni II & $12.89(0.10)$ & & & & & $\mathrm{N} \mathrm{I}$ & $<14.30$ \\
\hline & & & & Mn II & $12.04(0.03)$ & & & & & Mg II & $14.99(0.14)$ \\
\hline & & & & Si II & $15.06(0.01)$ & 5 & 1.776859 & +53 & $5.5(0.3)$ & Fe II & $13.63(0.05)$ \\
\hline & & & & Ti II & $11.34(0.10)$ & & & & & Zn II & $11.43(0.04)$ \\
\hline & & & & S II & $14.88(0.12)$ & & & & & Cr II & $12.12(0.05)$ \\
\hline & & & & P II & $13.11(0.04)$ & & & & & $\mathrm{Ni}$ II & $12.58(0.05)$ \\
\hline & & & & N I & $<15.16$ & & & & & Mn II & $11.33(0.04)$ \\
\hline & & & & $\mathrm{Cl} \mathrm{I}$ & $12.90(0.03)$ & & & & & Si II & $14.05(0.03)$ \\
\hline & & & & Mg II & $15.27(0.12)$ & & & & & $S_{\text {II }}$ & $14.05(0.10)$ \\
\hline \multirow[t]{11}{*}{3} & 1.776538 & +18 & $6.0(0.3)$ & Fe II & $13.46(0.04)$ & & & & & P II & $12.00(0.30)$ \\
\hline & & & & Zn II & $11.81(0.02)$ & & & & & N I & $<12.82$ \\
\hline & & & & Cr II & $12.07(0.04)$ & & & & & Mg II & $14.31(0.26)$ \\
\hline & & & & Ni II & $12.27(0.10)$ & 6 & 1.776964 & +64 & $9.4(0.9)$ & $\mathrm{Fe} I I$ & $13.23(0.07)$ \\
\hline & & & & Mn II & $11.53(0.02)$ & & & & & Si II & $13.92(0.05)$ \\
\hline & & & & Si II & $14.40(0.01)$ & & & & & $\mathrm{NI}_{\mathrm{I}}$ & $<13.33$ \\
\hline & & & & S II & $14.27(0.07)$ & & & & & & \\
\hline & & & & $\mathrm{P}_{\text {II }}$ & $12.19(0.25)$ & & & & & & \\
\hline & & & & $\mathrm{NI}$ & $<13.60$ & & & & & & \\
\hline & & & & $\mathrm{Cl} \mathrm{I}$ & $12.50(0.06)$ & & & & & & \\
\hline & & & & Mg II & $14.52(0.19)$ & & & & & & \\
\hline 1 & 1.776299 & -8 & $15.0(1.0)$ & $\overline{M g} \mathrm{I}$ & $11.72(0.07)$ & 4 & 1.776684 & +34 & $8.6(0.4)$ & Mg I & $11.83(0.07)$ \\
\hline \multirow[t]{2}{*}{2} & 1.776365 & -0.5 & $6.0(0.2)$ & $\mathrm{Mg}_{\mathrm{I}}$ & $12.08(0.03)$ & 5 & 1.776814 & +48 & 9.1(1.1) & $\mathrm{Mg}_{\mathrm{I}}$ & $11.85(0.07)$ \\
\hline & & & & CI & $13.12(0.02)$ & 6 & 1.776947 & +62 & $3.0(1.3)$ & $\mathrm{Mg}_{\mathrm{I}}$ & $11.39(0.05)$ \\
\hline \multirow[t]{2}{*}{3} & 1.776523 & +17 & $3.4(0.3)$ & Mg I & $11.77(0.01)$ & & & & & & \\
\hline & & & & C I & $12.72(0.02)$ & & & & & & \\
\hline \multicolumn{12}{|c|}{ Intermediate-ion transitions } \\
\hline 1 & 1.776036 & -36 & $8.4(0.4)$ & Al III & $12.03(0.02)$ & 4 & 1.776694 & +35 & $15.1(1.4)$ & $\mathrm{Al}$ III & $12.49(0.04)$ \\
\hline 2 & 1.776300 & -7 & $8.1(1.1)$ & $\mathrm{Al}$ III & $12.29(0.13)$ & 5 & 1.776866 & +54 & $7.2(0.5)$ & Al III & $12.16(0.06)$ \\
\hline 3 & 1.776414 & +5 & $7.8(1.4)$ & $\mathrm{Al}$ III & $12.29(0.14)$ & & & & & & \\
\hline
\end{tabular}

${ }^{*}$ Velocity relative to $z=1.776370$.

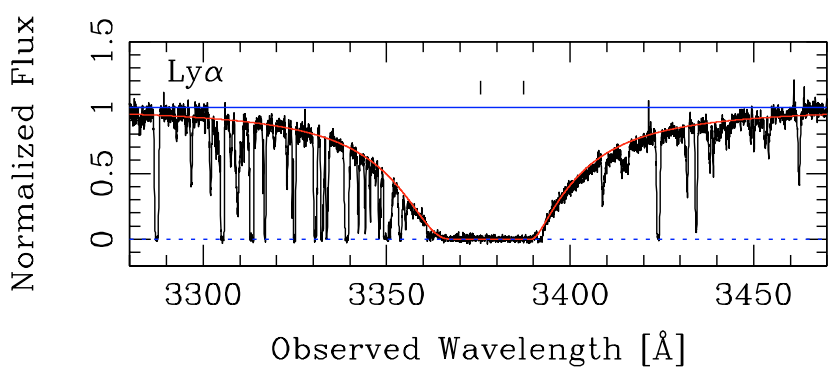

Fig. 3. Same as Fig. 1 but for Q1331+17. The vertical bars correspond to the wavelength centroids of the components used for the best fit, $z=1.776724$ and $z=1.786345$, referring to the DLA system and an additional absorber, respectively. The measured H I column densities $\operatorname{are} \log N(\mathrm{HI})=21.14 \pm 0.08$ and $19.80 \pm 0.10$, respectively. in DLAs (the ionization potential of $\mathrm{Cl}^{0}$ is $13.01 \mathrm{eV}$ which is lower than that of hydrogen). The $\mathrm{Cl}$ II column density cannot unfortunately be determined in this DLA system due to the low rest-wavelengths of the $\mathrm{Cl}$ II lines.

The N I column density is not easy to derive in this DLA. First, given the high $\mathrm{H}$ I column density of this DLA system, the NI triplet at $\lambda_{\text {rest }} \sim 1200 \AA$ is blended with the blue damping wing of the DLA Ly $\alpha$ absorption line. We thus have to renormalize the spectral region covering the N I $\lambda 1200$ triplet with the fit of the Ly $\alpha$ damping wing profile, before fitting the $\mathrm{N}$ I lines. Second, it is difficult to determine whether the derived N I column density is a lower or an upper limit. Indeed, the component 2 of all the detected N I lines is saturated, even in the weakest N I transition at $\lambda_{\text {rest }}=1134.98 \AA$. Thus, we are 

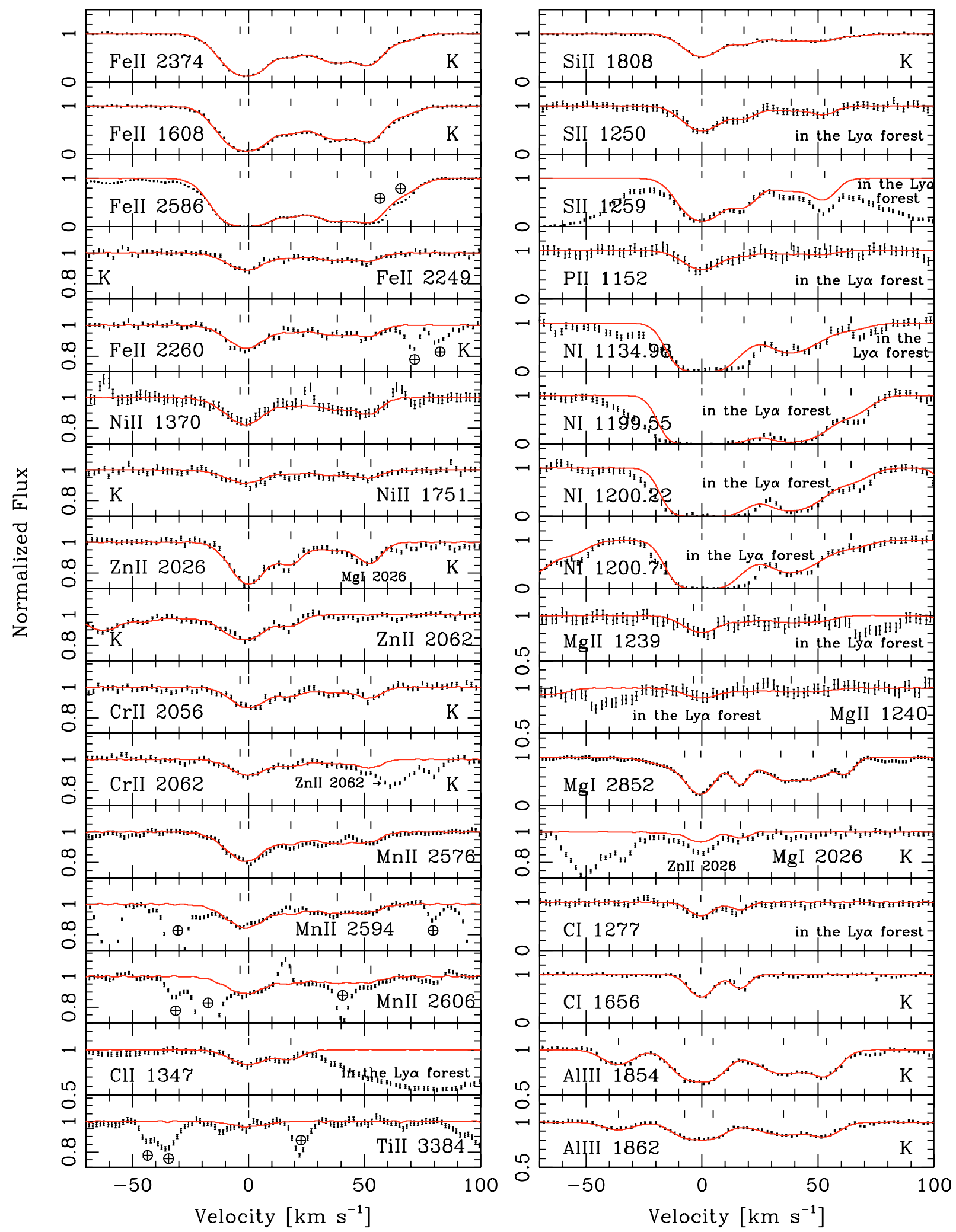

Fig. 4. Same as Fig. 2 for the DLA toward Q1331+17. The zero velocity is fixed at $z=1.776370$.

inclined to assume that the derived column density is a lower limit. But, when looking carefully at the N I line profiles, they all show a stronger component 4 than the component 5 , on the opposite to what is observed in other low-ionization transition profiles. This suggests that the N I lines are likely blended with
Ly $\alpha$ forest absorptions. Therefore, we finally assume that the derived $\mathrm{N}$ I column density is an upper limit.

While fitting the Ly $\alpha$ damping line, we found it necessary to include, besides the contribution of the DLA at $z=1.776724$ corresponding to the redshift of the metal-line component 4 , 


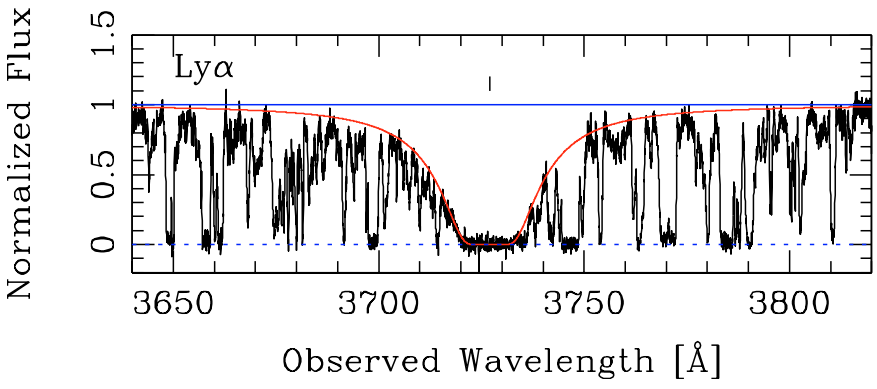

Fig. 5. Same as Fig. 1 but for Q2231-00. The vertical bar corresponds to the wavelength centroid of the component used for the best fit left as a free parameter, $z=2.065774$. The measured H I column density is $\log N(\mathrm{HI})=20.53 \pm 0.08$.

a second absorber shifted by about $1079 \mathrm{~km} \mathrm{~s}^{-1}$ redwards of the DLA system (see Fig. 3). The redshift of this second absorber, $z_{\mathrm{abs}}=1.786$, is accurately determined thanks to the detections of several associated metal-lines (see also Lopez \& Ellison 2003). The derived H I column densities of the DLA and the second absorber are $\log N(\mathrm{HI})=21.14 \pm 0.08$ and $19.80 \pm 0.10$, respectively. They confirm the previous measurements made by Pettini et al. (1994).

\section{3. $Q 2231-00, Z_{a b s}=2.066$}

This DLA system was previously observed and analyzed by Lu et al. (1996) and Prochaska \& Wolfe (1999), and recently completed by Prochaska et al. (2001). Throughout their analysis they adopted the H I column density measured by Lu \& Wolfe (1994). We obtain here a new value for the $\log N(\mathrm{HI})$ of $20.53 \pm 0.08$ from the Ly $\alpha$ damping line observed in the UVES spectra (see Fig. 5). The fit was performed by fixing the $b$-value at $20 \mathrm{~km} \mathrm{~s}^{-1}$ and by leaving the redshift as a free parameter.

The UVES spectra provide column density measurements of the $\mathrm{S}^{+}, \mathrm{P}^{+}, \mathrm{N}^{0}, \mathrm{Mn}^{+}$and $\mathrm{Mg}^{0}$ ions (see Fig. 6), and confirm the $N\left(\mathrm{Si}^{+}\right), N\left(\mathrm{Zn}^{+}\right), N\left(\mathrm{Cr}^{+}\right)$and $N\left(\mathrm{Ni}^{+}\right)$measurements of Prochaska \& Wolfe (1999). The fit of the Mg I $\lambda 2852$ line clearly shows that the contamination of $\mathrm{Zn}$ II $\lambda 2026$ by the $\mathrm{Mg}$ I $\lambda 2026$ profile is negligible. We note that we had to fit the $\mathrm{Mg}$ I profile with slightly different fitting parameters in comparison to the ones we have used for other low-ion metal lines (see Table 5). By combining the Fe II $\lambda 1608,1611$ lines detected in the HIRES spectra and the Fe II 12260, 2344, 2374 lines observed in the UVES spectra, we deduce a more accurate measurement of $N\left(\mathrm{Fe}^{+}\right)$. Finally, we obtain a revised value for $N$ (Ti II) of $12.66 \pm 0.08$ from the Ti II $\lambda 3067,3073$ lines. We succeed in deriving an upper limit on the column density of the intermediate-ion, Fe III, located in the Ly $\alpha$ forest, by using the Al III lines observed in the HIRES spectra to constrain the fitting parameters (see Fig. 6 and Table 5). The intermediate-ion lines show very similar profiles to the low-ion line profiles.

The low-ion line profiles are characterized by a relatively complex velocity structure composed of 11 components presented in Table 5 and spread over $180 \mathrm{~km} \mathrm{~s}^{-1}$ in velocity space. But, only the 5 strongest components, the components 4,5 , 9, 10 and 11, are detected in the weak metal-lines. They contain only about $75 \%$ of the total column density obtained by summing the contributions of the 11 components. This shows that when computing the abundance ratios $[X / Y]$, it is necessary to consider only the column densities of the velocity components detected in both the $X$ and $Y$ profiles to avoid under/overestimations of the relative abundances.

An additional difficulty which appears in absorption systems with complex metal-line profiles is that the probability of blending in the Ly $\alpha$ forest is higher over a larger velocity interval. For this reason it is more sensible to consider the measured $\mathrm{P}^{+}$and $\mathrm{N}^{0}$ column densities as upper limits, the $\mathrm{P}$ II and $\mathrm{N}$ I lines being located in a region of the Ly $\alpha$ forest with numerous absorption lines (see Fig. 6). The $\mathrm{S}^{+}$column density is a borderline case, and unfortunately only the $\mathrm{S}$ II $\lambda 1253$ line from the $\mathrm{S}^{+}$triplet can be used to determine $N\left(\mathrm{~S}^{+}\right)$, the two other lines are heavily blended with Ly $\alpha$ clouds. We consider the measured $N\left(\mathrm{~S}^{+}\right)$as a value, assuming that the adopted error on $N\left(\mathrm{~S}^{+}\right)$covers the possible $\mathrm{H}$ I blendings.

\section{4. $Q 2343+12, Z_{a b s}=2.431$}

This DLA system has first been studied by Sargent et al. (1988). The chemical abundance measurements obtained from HIRES spectra were mainly used in statistical samples (e.g. Lu et al. 1998; Prochaska \& Wolfe 1998; Vladilo et al. 2003). Recently, D'Odorico et al. (2002) and Vladilo et al. (2003) reported some additional abundance measurements from UVES spectra. We present here the first complete set of elemental abundances of this DLA.

From our UVES spectra, we measured the column densities of O I, Ar I, N I, Fe II, Zn II, Cr II and Mn II, and obtained an upper limit on the column density of P II contaminated by the Ly $\alpha$ clouds. The metal absorption profiles are characterized by an extremely complex velocity structure extended over $350 \mathrm{~km} \mathrm{~s}^{-1}$ in velocity space and composed of 23 components (see Fig. 8 and Table 6). The dominant feature is the group of 5 components around $v \sim+120 \mathrm{~km} \mathrm{~s}^{-1}$, with the components 18-20 being the strongest ones. These components are heavily saturated in strong metal-lines, but are the only components detected in weak metal-lines. They contain about $62 \%$ of the total column density.

The Ly $\alpha$ line is outside our UVES wavelength coverage, therefore we adopt the H I column density measurement $(\log N(\mathrm{HI})=20.35 \pm 0.05)$ obtained by D'Odorico et al. (2002). Figure 7 shows the fit of the $\operatorname{Ly} \beta$ line which confirms this $N\left(\mathrm{H}^{0}\right)$ measurement. This relatively low $\mathrm{H}^{0}$ column density and the presence of a strong N II $\lambda 1083$ line as well as of other intermediate-ion lines - Al III $\lambda 1854$, Fe III $\lambda 1122$ and S III $\lambda 1012$ - with very similar profiles to the low-ion metal line profiles (see Fig. 8 and Table 7) suggest that some photoionization corrections may be required in this DLA system. A discussion on the photoionization corrections is presented in Sect. 4.

\section{Photoionization corrections}

The photoionization effects have to be carefully examined in absorption metal-line systems, when the main goal is to provide a complete and unbiased interpretation of the chemical 
Table 5. Component structure of the $z_{\mathrm{abs}}=2.066$ DLA system toward Q2231-00.

\begin{tabular}{|c|c|c|c|c|c|c|c|c|c|c|c|}
\hline No. & $z_{\mathrm{abs}}$ & $\begin{array}{c}v_{\text {rel }}^{*} \\
\mathrm{~km} \mathrm{~s}^{-1}\end{array}$ & $\begin{array}{c}b\left(\sigma_{b}\right) \\
\mathrm{km} \mathrm{s}^{-1}\end{array}$ & Ion & $\log N\left(\sigma_{\log N}\right)$ & No. & $z_{\mathrm{abs}}$ & $\begin{array}{c}v_{\text {rel }}^{*} \\
\mathrm{~km} \mathrm{~s}^{-1}\end{array}$ & $\begin{array}{c}b\left(\sigma_{b}\right) \\
\mathrm{km} \mathrm{s}^{-1}\end{array}$ & Ion & $\log N\left(\sigma_{\log N}\right)$ \\
\hline \multicolumn{12}{|c|}{ Low-ion transitions } \\
\hline \multirow[t]{2}{*}{1} & 2.064746 & -138 & $6.6(0.5)$ & Fe II & $13.06(0.04)$ & 8 & 2.065887 & -27 & $4.9(0.1)$ & Fe II & $13.41(0.02)$ \\
\hline & & & & Si II & $13.53(0.12)$ & & & & & Si II & $13.87(0.01)$ \\
\hline \multirow[t]{2}{*}{2} & 2.064897 & -123 & $9.0(0.7)$ & Fe II & $13.20(0.07)$ & & & & & S II & $13.80(0.26)$ \\
\hline & & & & Si II & $13.79(0.06)$ & & & & & Ni & $13.59(0.22)$ \\
\hline \multirow[t]{2}{*}{3} & 2.065087 & -105 & $13.8(1.4)$ & Fe II & $13.58(0.04)$ & 9 & 2.066072 & -9 & $5.5(0.4)$ & Fe II & $14.00(0.05)$ \\
\hline & & & & Si II & $13.97(0.10)$ & & & & & Zn II & $11.71(0.06)$ \\
\hline \multirow[t]{9}{*}{4} & 2.065266 & -88 & $8.6(0.2)$ & Fe II & $14.19(0.01)$ & & & & & Cr II & $12.12(0.08)$ \\
\hline & & & & Zn II & $11.63(0.04)$ & & & & & Ni II & $12.73(0.03)$ \\
\hline & & & & Cr II & $12.46(0.03)$ & & & & & Mn II & $11.92(0.05)$ \\
\hline & & & & Ni II & $13.04(0.10)$ & & & & & Si II & $14.59(0.04)$ \\
\hline & & & & Mn II & $11.97(0.03)$ & & & & & Ti II & $11.88(0.18)$ \\
\hline & & & & Si II & $14.57(0.04)$ & & & & & S II & $14.33(0.13)$ \\
\hline & & & & S II & $14.19(0.13)$ & & & & & $\mathrm{P}_{\text {II }}$ & $<12.81$ \\
\hline & & & & P II & $<12.90$ & & & & & N I & $14.43(0.06)$ \\
\hline & & & & N I & $13.95(0.11)$ & 10 & 2.066161 & 0 & $3.8(0.2)$ & Fe II & $14.26(0.03)$ \\
\hline \multirow[t]{9}{*}{5} & 2.065422 & -72 & $4.2(0.5)$ & $\mathrm{Fe}$ II & $13.59(0.03)$ & & & & & Zn II & $11.98(0.03)$ \\
\hline & & & & Zn II & $10.92(0.17)$ & & & & & Cr II & $12.60(0.02)$ \\
\hline & & & & Cr II & $11.77(0.13)$ & & & & & Ni II & $13.01(0.02)$ \\
\hline & & & & Ni II & $12.44(0.17)$ & & & & & Mn II & $12.16(0.03)$ \\
\hline & & & & Mn II & $11.46(0.07)$ & & & & & Si II & $14.83(0.03)$ \\
\hline & & & & Si II & $13.90(0.06)$ & & & & & Ti II & $12.58(0.06)$ \\
\hline & & & & $S_{\text {II }}$ & $13.87(0.16)$ & & & & & S II & $14.66(0.13)$ \\
\hline & & & & P II & $<12.32$ & & & & & P II & $<13.03$ \\
\hline & & & & $\mathrm{N}_{\mathrm{I}}$ & $13.22(0.03)$ & & & & & N I & $14.76(0.22)$ \\
\hline \multirow[t]{4}{*}{6} & 2.065546 & -60 & $6.4(0.2)$ & Fe II & $13.58(0.02)$ & 11 & 2.066275 & +11 & $5.4(0.1)$ & $\mathrm{Fe}$ II & $13.72(0.01)$ \\
\hline & & & & Si II & $13.83(0.08)$ & & & & & Cr II & $12.07(0.08)$ \\
\hline & & & & $S_{\text {II }}$ & $13.99(0.14)$ & & & & & Ni II & $12.70(0.04)$ \\
\hline & & & & N I & $13.25(0.13)$ & & & & & Mn II & $11.60(0.06)$ \\
\hline \multirow[t]{4}{*}{7} & 2.065698 & -45 & $4.7(0.3)$ & $\mathrm{Fe}$ II & $13.18(0.02)$ & & & & & Si II & $13.94(0.03)$ \\
\hline & & & & Si II & $13.57(0.01)$ & & & & & S II & $14.06(0.21)$ \\
\hline & & & & S II & $13.89(0.25)$ & & & & & P II & $<12.74$ \\
\hline & & & & $\mathrm{N} \mathrm{I}$ & $13.30(0.23)$ & & & & & $\mathrm{NI}$ & $13.50(0.23)$ \\
\hline 1 & 2.064788 & -134 & $7.6(3.0)$ & Mg I & $11.04(0.12)$ & 6 & 2.065903 & -25 & $7.0(0.7)$ & $\overline{M g} \mathrm{I}$ & $11.36(0.03)$ \\
\hline 2 & 2.064981 & -115 & $7.9(2.1)$ & $\mathrm{Mg}_{\mathrm{I}}$ & $11.12(0.09)$ & 7 & 2.066064 & -9 & $5.2(0.4)$ & $\operatorname{Mg}_{I}$ & $11.72(0.05)$ \\
\hline 3 & 2.065233 & -91 & $8.8(0.5)$ & $\mathrm{Mg}_{\mathrm{I}}$ & $11.87(0.03)$ & 8 & 2.066155 & -0.6 & 3.1(1.3) & $\operatorname{Mg}_{I}$ & $12.11(0.06)$ \\
\hline 4 & 2.065461 & -68 & $14.7(1.7)$ & $\mathrm{Mg}_{\mathrm{I}}$ & $11.65(0.05)$ & 9 & 2.066274 & +11 & $4.6(1.0)$ & $\mathrm{Mg}_{\mathrm{I}}$ & $11.59(0.06)$ \\
\hline 5 & 2.065692 & -46 & $3.0(1.0)$ & Mg I & $11.05(0.06)$ & & & & & & \\
\hline \multicolumn{12}{|c|}{ Intermediate-ion transitions } \\
\hline \multirow[t]{2}{*}{1} & 2.064758 & -137 & $3.0(1.2)$ & $\overline{\mathrm{Al} \text { III }}$ & $11.72(0.07)$ & 6 & 2.065648 & -50 & $10.7(2.3)$ & $\mathrm{Al}$ III & $11.88(0.07)$ \\
\hline & & & & Fe III & $<13.64$ & & & & & Fe III & $<13.83$ \\
\hline \multirow[t]{2}{*}{2} & 2.064909 & -122 & $6.6(1.5)$ & $\mathrm{Al} \mathrm{III}$ & $12.00(0.05)$ & 7 & 2.065925 & -23 & $8.5(2.7)$ & $\mathrm{Al}$ III & $12.10(0.10)$ \\
\hline & & & & Fe III & $<13.92$ & & & & & $\mathrm{Fe}$ III & $<13.83$ \\
\hline \multirow[t]{2}{*}{3} & 2.065123 & -101 & $8.5(2.3)$ & $\mathrm{Al} \mathrm{III}$ & $12.01(0.10)$ & 8 & 2.066076 & -8 & $3.3(1.0)$ & $\mathrm{Al}$ III & $12.04(0.19)$ \\
\hline & & & & Fe III & $<13.78$ & & & & & Fe III & $<13.59$ \\
\hline \multirow[t]{2}{*}{4} & 2.065274 & -87 & $6.5(0.7)$ & $\mathrm{Al}$ III & $12.50(0.03)$ & 9 & 2.066165 & 0 & $5.1(2.5)$ & Al III & $12.51(0.09)$ \\
\hline & & & & $\mathrm{Fe}$ III & $<13.59$ & & & & & $\mathrm{Fe}$ III & $<13.74$ \\
\hline \multirow[t]{2}{*}{5} & 2.065435 & -71 & $3.0(1.6)$ & $\mathrm{Al}$ III & $11.97(0.02)$ & 10 & 2.066290 & +13 & $4.8(1.3)$ & $\mathrm{Al}$ III & $11.96(0.13)$ \\
\hline & & & & $\mathrm{Fe}$ III & $<13.77$ & & & & & $\mathrm{Fe}$ III & $<13.59$ \\
\hline
\end{tabular}

${ }^{*}$ Velocity relative to $z=2.066161$.

abundance patterns. Indeed, as we are studying gas-phase abundances, a fraction of the gas may be ionized and we need to determine this fraction to be sure that we are measuring the intrinsic abundances. The dust depletion effects also affect the gas-phase abundance measurements. The manner we tackle this issue is described in the next section.
It is generally assumed that in the DLA systems the ionization fraction is low, so that the dominant ionization state in $\mathrm{HI}$ regions is the neutral one for elements with the first ionization potential $>13.6 \mathrm{eV}$ (e.g. $\mathrm{O}^{0}, \mathrm{~N}^{0}$ ) and the singly ionized one for elements with the first ionization potential $<13.6 \mathrm{eV}$ and the second $>13.6 \mathrm{eV}$ (e.g. $\mathrm{Fe}^{+}, \mathrm{Si}^{+}$). The reason for this is 
M. Dessauges-Zavadsky et al.: A comprehensive set of elemental abundances in DLAs
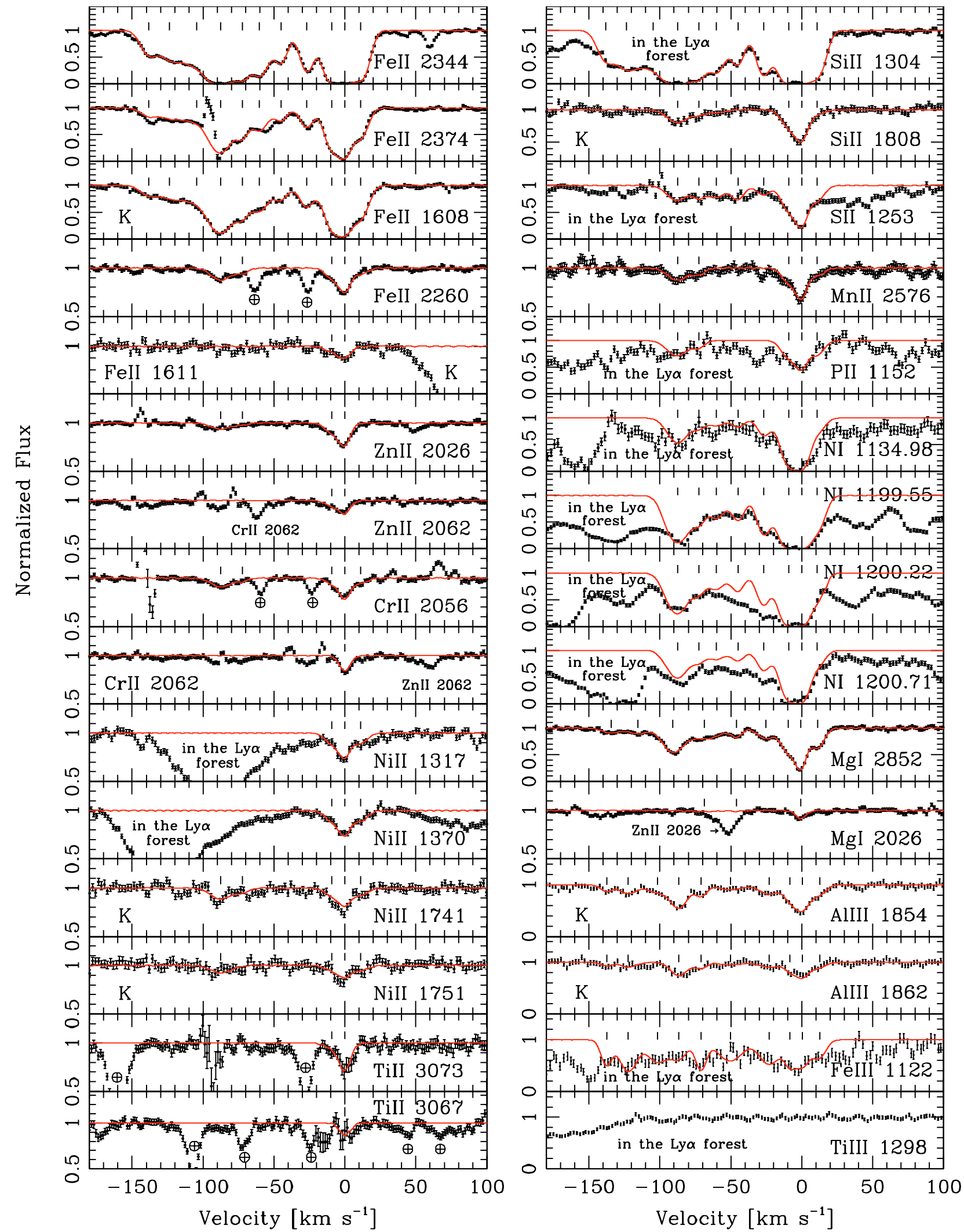

Fig. 6. Same as Fig. 2 for the DLA toward Q2231-00. The zero velocity is fixed at $z=2.066161$.

that the bulk of the $\mathrm{H}^{0}$ gas is self-shielded from $h v>13.6 \mathrm{eV}$ photons, but transparent to $h v<13.6 \mathrm{eV}$ photons. Under this assumption, the correction factors for ionization are small, and thus the column densities of low-ions are equal to the column densities of elements, e.g. $N\left(\mathrm{Si}^{+}\right) \simeq N(\mathrm{Si})$. However, the detection of intermediate-ionization transitions, like $\mathrm{Al}^{++}$, in the DLA systems provided doubts on the ionization levels in DLAs. The ionization potential of $\mathrm{Al}^{+}$is $18.8 \mathrm{eV}$, i.e. greater 
Table 6. Component structure of the $z_{\mathrm{abs}}=2.431$ DLA system toward Q2343+12.

\begin{tabular}{|c|c|c|c|c|c|c|c|c|c|c|c|}
\hline No. & $\bar{Z}$ Zabs & $\begin{array}{c}v_{\mathrm{rel}}^{*} \\
\mathrm{~km} \mathrm{~s}^{-1}\end{array}$ & $\begin{array}{c}c b\left(\sigma_{b}\right) \\
\mathrm{km} \mathrm{s}^{-1}\end{array}$ & Ion & $\overline{\log N\left(\sigma_{\log N}\right)}$ & No. & $z_{\mathrm{abs}}$ & $\begin{array}{c}v_{\text {rel }}^{*} \\
\mathrm{~km} \mathrm{~s}^{-1}\end{array}$ & $\begin{array}{c}b\left(\sigma_{b}\right) \\
\mathrm{km} \mathrm{s}^{-1}\end{array}$ & Ion & $\overline{\overline{\log N\left(\sigma_{\log N}\right)}}$ \\
\hline \multicolumn{12}{|c|}{ Low-ion transitions } \\
\hline \multirow[t]{3}{*}{1} & 2.428181 & -154 & $3.0(1.0)$ & Fe II & $12.44(0.03)$ & 15 & 2.430674 & +64 & $2.8(0.2)$ & Fe II & $12.38(0.03)$ \\
\hline & & & & O I & $14.38(0.03)$ & & & & & O I & $13.93(0.05)$ \\
\hline & & & & Si II & $13.59(0.02)$ & & & & & Si II & $13.13(0.01)$ \\
\hline \multirow[t]{3}{*}{2} & 2.428277 & -146 & $3.0(0.9)$ & Fe II & $12.94(0.02)$ & 16 & 2.430808 & +76 & $3.2(0.1)$ & $\mathrm{Fe}$ II & $12.65(0.02)$ \\
\hline & & & & O I & $14.98(0.04)$ & & & & & O I & $14.37(0.03)$ \\
\hline & & & & Si II & $13.81(0.06)$ & & & & & Si II & $13.32(0.01)$ \\
\hline \multirow[t]{3}{*}{3} & 2.428383 & -136 & $4.1(0.3)$ & Fe II & $13.18(0.01)$ & 17 & 2.431018 & +94 & $8.3(0.4)$ & $\mathrm{Fe}$ II & $13.45(0.02)$ \\
\hline & & & & O I & $14.52(0.02)$ & & & & & O I & $15.27(0.05)$ \\
\hline & & & & Si II & $14.12(0.06)$ & & & & & Si II & $14.01(0.09)$ \\
\hline \multirow[t]{3}{*}{4} & 2.428499 & -126 & $4.4(0.5)$ & Fe II & $12.49(0.04)$ & 18 & 2.431157 & +106 & $5.1(0.4)$ & $\mathrm{Fe}$ II & $13.98(0.04)$ \\
\hline & & & & O I & $13.30(0.19)$ & & & & & $\mathrm{Zn}$ II & $11.72(0.03)$ \\
\hline & & & & Si II & $13.37(0.01)$ & & & & & Cr II & $12.37(0.03)$ \\
\hline \multirow[t]{3}{*}{5} & 2.428749 & -104 & $8.5(0.3)$ & Fe II & $12.74(0.01)$ & & & & & Mn II & $11.82(0.04)$ \\
\hline & & & & O I & $14.51(0.02)$ & & & & & O I & $15.76(0.06)$ \\
\hline & & & & Si II & $13.08(0.05)$ & & & & & Si II & $14.55(0.03)$ \\
\hline \multirow[t]{3}{*}{6} & 2.428884 & -93 & $3.0(1.0)$ & Fe II & $12.68(0.02)$ & & & & & Ar I & $12.85(0.18)$ \\
\hline & & & & O I & $14.29(0.03)$ & & & & & $\mathrm{NI}_{\mathrm{I}}$ & $14.31(0.22)$ \\
\hline & & & & Si II & $12.95(0.02)$ & & & & & P II & $<12.67$ \\
\hline \multirow[t]{3}{*}{7} & 2.429009 & -82 & $5.9(0.4)$ & Fe II & $12.45(0.02)$ & 19 & 2.431288 & +118 & $6.2(0.6)$ & $\mathrm{Fe}$ II & $14.09(0.03)$ \\
\hline & & & & O I & $14.16(0.03)$ & & & & & $\mathrm{Zn}$ II & $11.87(0.02)$ \\
\hline & & & & Si II & $12.87(0.02)$ & & & & & Cr II & $12.38(0.03)$ \\
\hline \multirow[t]{3}{*}{8} & 2.429249 & -61 & $5.1(0.4)$ & Fe II & $12.50(0.02)$ & & & & & Mn II & $11.89(0.03)$ \\
\hline & & & & O I & $14.15(0.03)$ & & & & & O I & $15.90(0.05)$ \\
\hline & & & & Si II & $13.14(0.05)$ & & & & & Si II & $14.71(0.02)$ \\
\hline \multirow[t]{3}{*}{9} & 2.429355 & -51 & $3.0(0.8)$ & Fe II & $12.85(0.03)$ & & & & & Ar I & $12.86(0.10)$ \\
\hline & & & & O I & $14.54(0.02)$ & & & & & $\mathrm{NI}_{\mathrm{I}}$ & $14.32(0.15)$ \\
\hline & & & & Si II & $13.71(0.02)$ & & & & & $P_{\text {II }}$ & $<12.55$ \\
\hline \multirow[t]{3}{*}{10} & 2.429500 & -39 & $5.2(0.2)$ & Fe II & $12.65(0.01)$ & 20 & 2.431439 & +131 & $4.7(0.4)$ & $\mathrm{Fe}$ II & $13.82(0.03)$ \\
\hline & & & & O I & $14.22(0.03)$ & & & & & $\mathrm{Zn}$ II & $11.67(0.03)$ \\
\hline & & & & Si II & $13.28(0.09)$ & & & & & Cr II & $12.20(0.05)$ \\
\hline \multirow[t]{3}{*}{11} & 2.429702 & -21 & $4.4(0.5)$ & Fe II & $12.20(0.02)$ & & & & & Mn II & $11.64(0.05)$ \\
\hline & & & & O I & $13.97(0.05)$ & & & & & O I & $15.80(0.05)$ \\
\hline & & & & Si II & $12.81(0.06)$ & & & & & Si II & $14.54(0.03)$ \\
\hline \multirow[t]{3}{*}{12} & 2.429942 & 0 & $7.3(0.2)$ & Fe II & $13.28(0.01)$ & & & & & ArI & $12.44(0.22)$ \\
\hline & & & & O I & $14.89(0.01)$ & & & & & $\mathrm{NI}_{\mathrm{I}}$ & $13.58(0.25)$ \\
\hline & & & & Si II & $13.73(0.11)$ & & & & & P II & $<12.45$ \\
\hline \multirow[t]{3}{*}{13} & 2.430086 & +13 & $9.6(0.8)$ & Fe II & $12.86(0.06)$ & 21 & 2.431570 & +142 & $5.7(0.2)$ & $\mathrm{Fe}$ II & $13.48(0.04)$ \\
\hline & & & & O I & $14.59(0.02)$ & & & & & O I & $15.09(0.07)$ \\
\hline & & & & Si II & $13.33(0.01)$ & & & & & Si II & $13.76(0.16)$ \\
\hline \multirow[t]{4}{*}{14} & 2.430394 & +40 & $9.7(0.2)$ & Fe II & $13.19(0.01)$ & 22 & 2.431826 & +165 & $6.9(0.1)$ & Fe II & $13.02(0.01)$ \\
\hline & & & & O I & $14.75(0.01)$ & & & & & Si II & $13.77(0.01)$ \\
\hline & & & & Si II & $13.68(0.01)$ & 23 & 2.432126 & +191 & $6.9(0.5)$ & Fe II & $12.27(0.03)$ \\
\hline & & & & & & & & & & Si II & $12.58(0.03)$ \\
\hline
\end{tabular}

* Velocity relative to $z=2.429942$

than that of hydrogen, therefore $\mathrm{Al}^{++}$is likely present in ionized and not in neutral gas, since photons with $h v>18.8 \mathrm{eV}$ cannot so easily penetrate gas clouds with large H I column densities. In addition, the observations show a good correlation between the velocity structures of Al III and singly ionized and neutral species (e.g. Lu et al. 1996; Prochaska \& Wolfe 1999; Wolfe \& Prochaska 2000). To explain the similarity between the Al III and low-ionization species line profiles, Howk \& Sembach (1999) and Izotov \& Thuan (1999) proposed that these lines originate in the same ionized region or in a mix of neutral and ionized clouds, and stressed the importance of abundance corrections for ionization effects. Furthermore, indirect arguments to consider ionized gas in DLAs came from studies of the warm ionized medium in the Milky Way and other nearby galaxies (Howk et al. 1999; Sembach et al. 2000; Jenkins et al. 2000).

Several authors have investigated the ionization effects in the DLA systems (Viegas 1995; Howk \& Sembach 1999; Izotov et al. 2001; Vladilo et al. 2001). Although the different approaches used to deal with the problem of photoionization in DLAs have led to slightly different conclusions, it was generally found that ionization corrections in DLAs are negligible, 
Table 7. Component structure of the $z_{\mathrm{abs}}=2.431$ DLA system toward Q2343+12 - continued.

\begin{tabular}{|c|c|c|c|c|c|c|c|c|c|c|c|}
\hline No. & $z_{\mathrm{abs}}$ & $\begin{array}{c}v_{\text {rel }}^{*} \\
\mathrm{~km} \mathrm{~s}^{-1}\end{array}$ & $\begin{array}{c}b\left(\sigma_{b}\right) \\
\mathrm{km} \mathrm{s}^{-1}\end{array}$ & Ion & $\log N\left(\sigma_{\log N}\right)$ & No. & $z_{\mathrm{abs}}$ & $\begin{array}{c}v_{\text {rel }}^{*} \\
\mathrm{~km} \mathrm{~s}^{-1}\end{array}$ & $\begin{array}{c}b\left(\sigma_{b}\right) \\
\mathrm{km} \mathrm{s}^{-1}\end{array}$ & Ion & $\log N\left(\sigma_{\log N}\right)$ \\
\hline \multicolumn{12}{|c|}{ Intermediate-ion transitions } \\
\hline \multirow[t]{4}{*}{1} & 2.428178 & -154 & $6.5(2.2)$ & Al III & $11.93(0.25)$ & 7 & 2.430392 & +39 & $10.8(0.5)$ & Al III & $11.92(0.03)$ \\
\hline & & & & Fe III & $13.06(0.06)$ & & & & & Fe III & $<13.40$ \\
\hline & & & & N II & $13.99(0.01)$ & & & & & N II & $13.66(0.02)$ \\
\hline & & & & S III & $<14.17$ & & & & & S III & $<13.84$ \\
\hline \multirow[t]{4}{*}{2} & 2.428288 & -144 & $3.6(1.6)$ & Al III & $11.90(0.27)$ & 8 & 2.430696 & +66 & $10.8(0.4)$ & $\mathrm{Al}$ III & $11.72(0.05)$ \\
\hline & & & & Fe III & $13.19(0.04)$ & & & & & N II & $13.71(0.01)$ \\
\hline & & & & N II & $14.48(0.21)$ & & & & & S III & $<13.90$ \\
\hline & & & & S III & $<13.56$ & 9 & 2.431065 & +98 & $15.0(0.2)$ & $\mathrm{Al}$ III & $12.08(0.26)$ \\
\hline \multirow[t]{4}{*}{3} & 2.428426 & -133 & $9.3(0.6)$ & Al III & $12.59(0.03)$ & & & & & N II & $14.04(0.01)$ \\
\hline & & & & Fe III & $13.54(0.02)$ & & & & & S III & $<14.35$ \\
\hline & & & & N II & $14.36(0.01)$ & 10 & 2.431185 & +109 & $8.9(1.0)$ & $\mathrm{Al}$ III & $12.45(0.08)$ \\
\hline & & & & S III & $<14.22$ & & & & & N II & $>14.25$ \\
\hline \multirow[t]{4}{*}{4} & 2.429358 & -51 & $8.3(0.5)$ & $\mathrm{Al}$ III & $11.51(0.07)$ & & & & & S III & $<13.71$ \\
\hline & & & & Fe III & $<10.00$ & 11 & 2.431323 & +121 & $6.7(0.9)$ & Al III & $12.39(0.08)$ \\
\hline & & & & N II & $13.89(0.04)$ & & & & & N II & $>14.11$ \\
\hline & & & & S III & $<13.97$ & & & & & S III & $<14.36$ \\
\hline \multirow[t]{4}{*}{5} & 2.429487 & -40 & $19.5(1.3)$ & Al III & $11.83(0.05)$ & 12 & 2.431443 & +131 & $3.0(1.5)$ & $\mathrm{Al}$ III & $11.92(0.08)$ \\
\hline & & & & Fe III & $<14.09$ & & & & & N II & $>13.66$ \\
\hline & & & & N II & $13.80(0.04)$ & & & & & S III & $<13.45$ \\
\hline & & & & S III & $<13.62$ & 13 & 2.431545 & +140 & $10.5(1.5)$ & $\mathrm{Al}$ III & $12.23(0.06)$ \\
\hline \multirow[t]{6}{*}{6} & 2.429995 & +5 & $14.4(0.8)$ & Al III & $12.09(0.02)$ & & & & & N II & $>14.27$ \\
\hline & & & & Fe III & $<13.36$ & & & & & S III & $<14.59$ \\
\hline & & & & N II & $13.71(0.02)$ & 14 & 2.431849 & +167 & $7.7(0.2)$ & $\mathrm{Al} \mathrm{III}$ & $11.72(0.04)$ \\
\hline & & & & S III & $<13.79$ & & & & & N II & $14.01(0.01)$ \\
\hline & & & & & & & & & & S III & $<14.34$ \\
\hline & & & & & & 15 & 2.432135 & +192 & $15.0(2.1)$ & N II & $13.72(0.01)$ \\
\hline
\end{tabular}

${ }^{*}$ Velocity relative to $z=2.429942$.

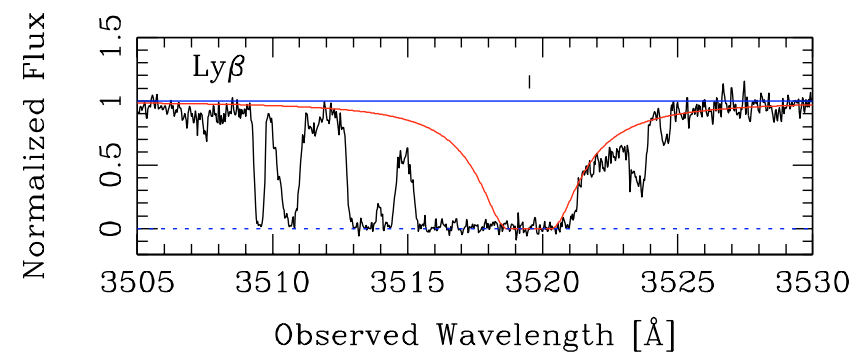

Fig. 7. Same as Fig. 1 but for Q2343+12. The Voigt fit was performed with the H I column density measured by D'Odorico et al. (2002, Sect. 3.2) from the $\operatorname{Ly} \alpha \operatorname{line}(\log N(\mathrm{HI})=20.35 \pm 0.05)$. The vertical bar corresponds to the wavelength centroid of the component used for the best fit by these authors, $z=2.43125$.

being below the measurement errors, except for some particular systems like the DLA toward GB 1759+7539 studied by Prochaska et al. (2002b). While the authors stress that this particular DLA system has several characteristics which separate it from the majority of DLAs, its properties highlight the importance of assessing the ionization state of each DLA system. The photoionization model computations are generally performed using the CLOUDY photoionization equilibrium software package (e.g. Ferland et al. 1998). To avoid computing heavy photoionization models, Prochaska et al. (2002b) defined a number of photoionization diagnostics which provides a qualitative "first-look" analysis of the ionization state in a DLA system and of the level of required ionization corrections. In the following sub-sections, we apply these photoionization diagnostics to each of the four DLAs studied and qualitatively evaluate the importance of ionization corrections.

\section{1. $Q 0100+13, Z_{a b s}=2.309$}

According to the anti-correlation between $\log N\left(\mathrm{H}^{0}\right)$ and $\log N\left(\mathrm{Al}^{++}\right) / N\left(\mathrm{Al}^{+}\right)$found by Vladilo et al. (2001), where the $\mathrm{Al}^{++} / \mathrm{Al}^{+}$column density ratio is assumed to be at a first approximation an indicator of the ionization level in the gas, the high H I column density, $\log N(\mathrm{HI})=21.37 \pm 0.08$, of this DLA system suggests that the ionization corrections are low. The following three additional and more reliable ionization indicators are at our disposal in this DLA system.

Thanks to the high efficiency of UVES in the blue, we have the spectral coverage for this DLA system of the rarely detected intermediate-ion transitions, Fe III and $\mathrm{N}_{\text {II }}$ at $\lambda_{\text {rest }}=$ 1122 and $1083 \AA$, respectively. The column density ratios $\mathrm{Fe}^{++} / \mathrm{Fe}^{+}$and $\mathrm{N}^{+} / \mathrm{N}^{0}$ are the most reliable ionization indicators, since the recombination coefficient of $\mathrm{Al}^{+}$is rather uncertain, being likely overestimated (Nussbaumer \& Storey 1986). The observed Fe III and N II lines show the same profiles as the low-ion profiles and are well fitted with the fitting parameters deduced for the low-ion lines. Located in the Ly $\alpha$ 

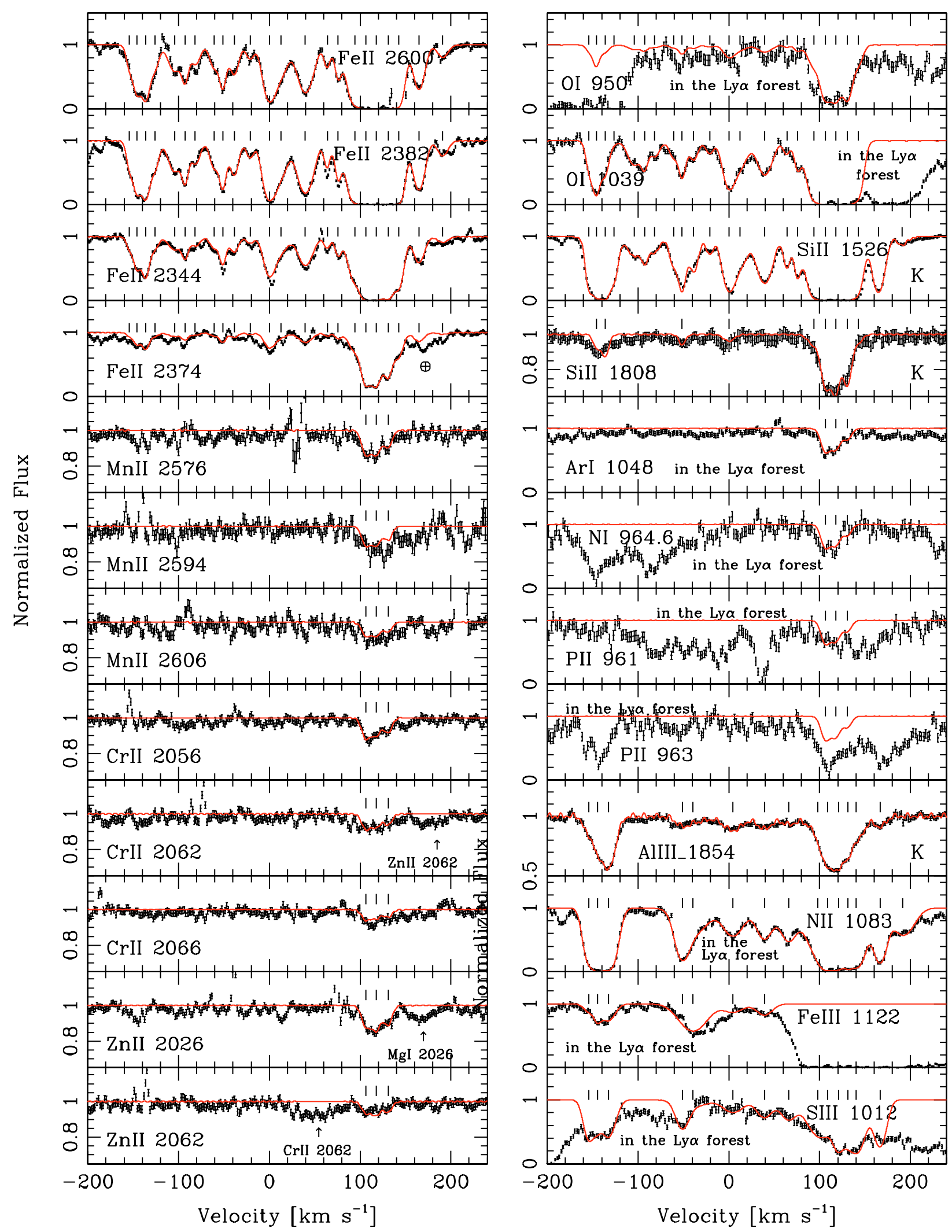

Fig. 8. Same as Fig. 2 for the DLA toward Q2343+12. The zero velocity is fixed at $z=2.429942$.

forest, one of the 2 components is clearly blended in both intermediate-ion lines (see Fig. 2), therefore we derived only upper limits on their column densities. These limits lead to $\log N\left(\mathrm{Fe}^{++}\right) / N\left(\mathrm{Fe}^{+}\right)<-1.54$ and $\log N\left(\mathrm{~N}^{+}\right) / N\left(\mathrm{~N}^{0}\right)<-1.24$.
According to the photoionization diagnostics established by Prochaska et al. (2002b), in a system where $\mathrm{Fe}^{++} / \mathrm{Fe}^{+}<$ $-1.60 \mathrm{dex}$ and $\mathrm{N}^{+} / \mathrm{N}^{0}<-1.00 \mathrm{dex}$, the ionization fraction, $x$, defined as the ratio of $\mathrm{H}^{+}$over $\left(\mathrm{H}^{0}+\mathrm{H}^{+}\right)$, is expected to be 
lower than $10 \%$. Thus, the measured $\mathrm{Fe}^{++}$and $\mathrm{N}^{+}$column densities are in agreement with the expectations for a DLA with a H I column density higher than $10^{21} \mathrm{~cm}^{-2}$.

Another ionization indicator that we can consider in this system is argon. Indeed, Ar has a very high photoionization cross-section for photons with energy higher than $13.6 \mathrm{eV}$ which is about ten times higher than the one of $\mathrm{HI}$, and thus $\mathrm{Ar}$ is very sensitive to ionization effects (Sofia \& Jenkins 1998). The comparison of the Ar abundance with the abundance of another $\alpha$-element - O, S, or $\mathrm{Si}$ - provides indication on the ionization level in the gas. In this system, we measure $[\mathrm{Ar} / \mathrm{S}]=$ $-0.20 \pm 0.14$, and according to Prochaska et al. (2002b), an observed value of $\left[\mathrm{Ar}^{0} / \mathrm{S}^{+}\right]>-0.20$ dex indicates $x<10 \%$.

In summary, the high $\mathrm{HI}$ column density, the $\mathrm{Al}^{++} / \mathrm{Al}^{+}$, $\mathrm{Fe}^{++} / \mathrm{Fe}^{+}$and $\mathrm{N}^{+} / \mathrm{N}^{0}$ column density ratios, and the $\mathrm{Ar}^{0} / \mathrm{S}^{+}$ abundance ratio, all point to a low ionization fraction in this DLA system, and thus to low ionization corrections. The ionization corrections should indeed be lower than 0.1 dex for all the elements, except probably for Ar.

\section{2. $Q 1331+17, Z_{a b s}=1.776$}

This is another very high HI column density system with $\log N(\mathrm{HI})=21.14 \pm 0.08$. The high $\mathrm{H}$ I column density suggests that the ionization level is low in this DLA (Vladilo et al. 2001). However, we do not detect any very reliable ionization indicator in this system to confirm this statement. Only the $\mathrm{Al}^{++} / \mathrm{Al}^{+}$column density ratio is available for which we derive an upper limit of $<-0.76 \mathrm{dex}$ from the HIRES spectra. This limit indicates that the ionization corrections should be low in this system.

\section{3. $Q 2231-00, Z_{a b s}=2.066$}

Two ionization indicators are accessible in this DLA with a $\mathrm{H}$ I column density of $\log N(\mathrm{HI})=20.53 \pm 0.08$. The first one is the $\mathrm{Al}^{++} / \mathrm{Al}^{+}$column density ratio obtained from the HIRES spectra, and the second one is the upper limit on the $\mathrm{Fe}^{++} / \mathrm{Fe}^{+}$column density ratio obtained from the UVES spectra. However, the Al II line is so heavily saturated that no reliable $N\left(\mathrm{Al}^{+}\right)$lower limit can be deduced. We thus derive an estimation of the $\mathrm{Al}^{++} / \mathrm{Al}^{+}$column density ratio by using $\mathrm{Si}^{+}$as a proxy of $\mathrm{Al}^{+}$assuming the $\log N\left(\mathrm{Si}^{+}\right)$versus $\log N\left(\mathrm{Al}^{+}\right)$correlation found by Vladilo et al. (2001). We obtain $\log N\left(\mathrm{Al}^{++}\right) / N\left(\mathrm{Al}^{+}\right)=-0.76 \pm 0.19$. With regard to the second ionization indicator, the Fe III $\lambda 1122$ line is located in the $\operatorname{Ly} \alpha$ forest and is strongly blended with H I clouds. Consequently, the deduced $\mathrm{Fe}^{++}$column density upper limit is not stringent. We get $\log N\left(\mathrm{Fe}^{++}\right) / N\left(\mathrm{Fe}^{+}\right) \ll-0.09$.

Unfortunately the Ar I lines as well as the N II line are beyond the quasar flux cut-off, no other ionization indicator is hence accessible in this DLA system. If we trust the derived $\mathrm{Al}^{++} / \mathrm{Al}^{+}$column density ratio measurement, the ionization corrections should not be significant.

\section{4. $Q 2343+12, z_{a b s}=2.431$}

The low H I column density of $\log N(\mathrm{H} \mathrm{I})=20.35 \pm 0.05$ suggests that the ionization corrections might be relevant in this system. Several strong intermediate-ion lines with very similar profiles to the low-ion line profiles are observed in this system, namely Al III, Fe III, N II and S III (see Fig. 8). This is indeed a first indication that photoionization is playing an important role here. The second straightforward indication is provided by the $\mathrm{Ar} / \mathrm{O}$ and $\mathrm{Ar} / \mathrm{Si}$ abundance ratio measurements. They are very low in this system. We find $[\mathrm{Ar} / \mathrm{O}]=-0.72 \pm 0.17$ and $[\mathrm{Ar} / \mathrm{Si}]=-0.81 \pm 0.16$, in agreement with the measurements by Vladilo et al. (2003). The $\mathrm{Ar} / \mathrm{Si}$ abundance ratio in this DLA system is even lower than the [Ar/Si] abundance ratio of $-0.68 \pm 0.04$ measured in the DLA system toward GB $1759+7539$ by Prochaska et al. (2002b) whose analysis showed important ionization corrections.

The measured $\log N\left(\mathrm{Al}^{++}\right) / N\left(\mathrm{Al}^{+}\right)=-0.62 \pm 0.19$ obtained by taking $\mathrm{Si}^{+}$as a proxy of $\mathrm{Al}^{+}$according to the correlation identified by Vladilo et al. (2001), $\log N\left(\mathrm{Fe}^{++}\right) / N\left(\mathrm{Fe}^{+}\right)<0.37$ derived from the Fe III $\lambda 1122$ line which is only partially blended with Ly $\alpha$ clouds (in the components 4 and 5), and $\log N\left(\mathrm{~N}^{+}\right) / N\left(\mathrm{~N}^{0}\right)>-0.10$ confirm that the ionization corrections are important in this DLA system. Indeed, a system with $\mathrm{Fe}^{++} / \mathrm{Fe}^{+}>-1$ dex and $\mathrm{N}^{+} / \mathrm{N}^{0}>-0.2$ dex is expected to have an ionization fraction, $x$, higher than $50 \%$ (Prochaska et al. 2002b). We suspect that the ionization corrections in this DLA system are of similar magnitude as the ones of the DLA toward GB $1759+7539$, namely between 0.1 to $>0.5$ dex depending on the ion. Such high ionization corrections prevent us from deriving reliable chemical abundances in this DLA system. Therefore, we do not include this DLA in the following Sections where we discuss the chemical abundances of individual systems and make a detailed comparison with chemical evolution models.

\section{Chemical abundances}

The small sample of DLAs studied in this paper is unique, because by combining the UVES-VLT data with the HIRES-Keck data we could measure the abundances of up to 15 elements N, O, Mg, Al, Si, P, S, Cl, Ar, Ti, Cr, Mn, Fe, Ni, Zn. This contrasts with the majority of DLAs for which only a handful of elements ( $\mathrm{Si}, \mathrm{Fe}$, occasionally $\mathrm{Cr}, \mathrm{Zn}, \mathrm{Ni}$ ) is usually detected (e.g. Lu et al. 1996; Prochaska \& Wolfe 1999; Prochaska et al. 2001). This low amount of information on individual systems has until now severely limited the interpretation of the DLA abundance patterns.

\subsection{Dust content}

The interpretation of the elemental abundance patterns in DLAs is by far not straightforward, the principal difficulty is to disentangle the nucleosynthetic contributions from the dust depletion effects. Several pieces of evidence show that dust is indeed present in DLAs (e.g. Pei et al. 1991; Pettini et al. 1994; Hou et al. 2001; Prochaska \& Wolfe 2002). Therefore, as we are measuring gas-phase elemental abundances in DLAs, 
in presence of dust the observed abundances may not represent the intrinsic chemical composition of the system if part of the elements is removed from the gas to the solid phase, as it happens in the interstellar medium of our Galaxy (Savage $\&$ Sembach 1996). Consequently, the refractory elements (e.g. $\mathrm{Si}, \mathrm{Fe}, \mathrm{Cr}, \mathrm{Ni}$ ) preferentially incorporated into dust grains are not the best diagnostic elements and their relative ratios have to be cautiously interpreted, since their differential depletion can mimic the expected nucleosynthetic abundance patterns.

In Sect. 4 we have seen that at least the ionization effects are playing a negligible role in three out of the four DLAs studied in this paper. To constrain now the dust depletion effects, the access to a large number of elements is crucial, since elements with the same nucleosynthetic origin provide information on the dust depletion level in DLAs.

Fe-peak elements and $\mathrm{Zn}$. When comparing the absolute abundances of different iron-peak elements, $\mathrm{Cr}, \mathrm{Fe}, \mathrm{Ni}$, and of Zn measured in the DLAs toward Q0100+13, Q1331+17 and Q2231-00, we notice variations, while in the Galactic stars, Cr, $\mathrm{Fe}, \mathrm{Ni}$ and $\mathrm{Zn}$ track each other and have solar values relative to $\mathrm{Fe}$ (apart from $\mathrm{Zn}$ which seems to be enhanced by at maximum 0.1-0.2 dex relative to Fe, see Prochaska et al. 2000; Mishenina et al. 2002). These absolute abundance variations are in line with the variations observed in the Galactic ISM due to the differential depletion (e.g. Savage \& Sembach 1996), and are thus suggestive as being the result of a differential depletion present in the DLA systems. Hence, the measured [ $\mathrm{Zn} / \mathrm{Fe}, \mathrm{Cr}, \mathrm{Ni}]$ ratios, when assumed as being the result of the different degree of incorporation into dust grains of $\mathrm{Zn}$, a volatile element (almost undepleted), and of $\mathrm{Fe}, \mathrm{Cr}$ and $\mathrm{Ni}$, refractory elements, clearly show that dust depletion has to be seriously taken into account in the DLA systems toward Q1331+17 and Q2231-00, for which we find $[\mathrm{Zn} / \mathrm{Fe}]=+0.75 \pm 0.05$ and $+0.45 \pm 0.07$, respectively. The DLA toward Q1331+17, in particular, exhibits one of the largest dust depletion level of any DLA. On the contrary, the DLA toward Q0100+13 shows a low [Zn/Fe] ratio of $+0.25 \pm 0.04$, and thus requires negligible dust corrections.

Alpha-elements. Another similar way to highlight the presence of dust in the DLA systems is the comparison of the absolute abundances of different $\alpha$-elements. In the Galactic stars the $\alpha$-elements track more or less each other (within $\pm 0.10 \mathrm{dex}$ ). Thus, by comparing $\alpha$-elements of different dust depletion levels, their relative ratios when differing from the solar value are indicative of the presence of dust. In the DLA toward $\mathrm{Q} 0100+13$ we measure $[\mathrm{S} / \mathrm{Mg}]=-0.10 \pm 0.12$, where $\mathrm{Mg}$ is relatively strongly depleted in the Galactic ISM and S is a nonrefractory element (Savage \& Sembach 1996). This ratio is in agreement with the measured $[\mathrm{Zn} / \mathrm{Fe}]$ ratio, both of them suggest a small amount of dust in this DLA system. In the DLA toward $\mathrm{Q} 1331+17$ we measure $[\mathrm{S} / \mathrm{Si}]=+0.16 \pm 0.12$. Since $\mathrm{Si}$ is only a mildly refractory element (Savage \& Sembach 1996), the observed $\mathrm{S}$ overabundance relative to $\mathrm{Si}$ indicates important amount of dust. We also have the measurement of $[\mathrm{S} / \mathrm{Mg}]=-0.07 \pm 0.18$ in this DLA system. This abundance ratio rather indicates no presence of dust, but we ascribe this result to the weak accuracy of the Mg II column density measurement, which may be slightly overestimated due to some blends in the Ly $\alpha$ forest (see Paper I and Sect. 3.2). Finally, in the
DLA toward Q2231-00 we measure $[\mathrm{S} / \mathrm{Si}]=+0.21 \pm 0.16$ and $[\mathrm{Si} / \mathrm{Ti}]=-0.25 \pm 0.09$. The $[\mathrm{S} / \mathrm{Si}]$ ratio is in agreement with the conclusions derived from the $[\mathrm{Zn} / \mathrm{Fe}]$ ratio, which highlights the presence of dust in this DLA system. On the other hand, the measured undersolar [Si/Ti] ratio, while $\mathrm{Ti}$ is a strongly refractory element, does not confirm these findings. At a first glance, the undersolar [ $\mathrm{Si} / \mathrm{Ti}]$ ratio can only be the result of an overestimation of the Ti II column density.

\subsection{Dust corrections}

Three main approaches can be used to circumvent the problem of dust depletion in the studies of DLA abundance patterns. The first one consists in considering only the DLA systems with no or low dust depletion, $[\mathrm{Zn} / \mathrm{Cr}] \lesssim 0.3$ (Pettini et al. 2000; Molaro et al. 2000; Lopez et al. 2002). Indeed, if the amount of dust is small, the dust depletion levels are negligible. This is valid for the DLA toward Q0100+13, which has a low $[\mathrm{Zn} / \mathrm{Cr}]=+0.12 \pm 0.02$ ratio. However, for the two other DLAs, other solutions have to be found. The second approach consists in trying to quantify the dust depletion effects and provide dust corrections to the measured abundances. Several authors have considered different methods to compute the dust depletion corrections (e.g. Vladilo 1998; Savaglio et al. 2000). Recently, Vladilo (2002a,b) elaborated a new and more complete method for correcting for dust depletion. Finally, the third approach consists in focusing on non-refractory and mildly refractory elements, such as N, O, S, Ar, and Zn. In this way one has directly access to the intrinsic abundances of DLAs (Centurión et al. 2000; Molaro et al. 2000; Dessauges-Zavadsky et al. 2002b). This approach to tackle the problem of dust depletion is by far the most accurate one. It is applicable to all DLAs whatever their amount of dust, and is independent of any assumption on the properties of dust in DLAs and of errors that may be introduced by a complex dust correction procedure.

In our study we considered the second and the third approach to circumvent the problem of dust depletion. In Table 8 we summarize all the absolute abundances, and in Table 9 we summarize the relative abundances which are further used in the paper. Both the observed and dust-corrected values are reported. The dust corrections were performed using the method developed by Vladilo (2002a,b). This method groups together several dust correction models based on different assumptions. They are labeled E00, E11 when one assumes that the intrinsic $[\mathrm{Zn} / \mathrm{Fe}]$ ratio is equal to $+0.10 \mathrm{dex}$ and $\mathrm{S} 00, \mathrm{~S} 11$ when the intrinsic $[\mathrm{Zn} / \mathrm{Fe}]$ ratio is equal to $+0.00 \mathrm{dex}$ (see more details in Vladilo 2002a). The absolute abundances, $[\mathrm{X} / \mathrm{H}]$, were obtained by summing the contributions of all the components reported in Tables 3-6. The relative abundances, $[\mathrm{X} / \mathrm{Y}]$, were computed by considering only the column densities of the components detected both in the $\mathrm{X}$ and $\mathrm{Y}$ profiles. In this way one avoids an overestimation of abundances derived from strong metal-line profiles relative to the abundances derived from weaker metal-line profiles in which generally only the strongest components are detected. In the case of very weak lines, like the Ti II lines for instance, one can indeed underestimate the $[\mathrm{X} / \mathrm{Fe}]$ ratios by up to $0.3-0.4$ dex by considering the 
Table 8. Summary of the absolute abundances in the three DLA systems studied.

\begin{tabular}{|c|c|c|c|c|c|c|c|}
\hline \multicolumn{2}{|c|}{ Quasar } & \multicolumn{2}{|c|}{ Q0100+13 } & \multicolumn{2}{|c|}{ Q1331+17 } & \multicolumn{2}{|c|}{ Q2231-00 } \\
\hline \multirow{2}{*}{\multicolumn{2}{|c|}{$\begin{array}{c}z_{\mathrm{abs}} \\
\log N(\mathrm{H} \mathrm{I})\end{array}$}} & \multicolumn{2}{|c|}{2.309} & \multicolumn{2}{|c|}{1.776} & \multicolumn{2}{|c|}{2.066} \\
\hline & & \multicolumn{2}{|c|}{$21.37(0.08)$} & \multicolumn{2}{|c|}{$21.14(0.08)$} & \multicolumn{2}{|c|}{$20.53(0.08)$} \\
\hline $\log N(\mathrm{Zn}$ II $)$ & {$[\mathrm{Zn} / \mathrm{H}]_{\mathrm{obs}}$} & $12.47(0.01)$ & $-1.57(0.09)$ & $12.54(0.02)$ & $-1.27(0.09)$ & $12.30(0.05)$ & $-0.90(0.10)$ \\
\hline $\log N(\mathrm{Fe}$ II $)$ & {$[\mathrm{Fe} / \mathrm{H}]_{\mathrm{obs}}$} & $15.09(0.01)$ & $-1.78(0.08)$ & $14.63(0.03)$ & $-2.01(0.09)$ & $14.83(0.03)$ & $-1.20(0.09)$ \\
\hline & {$[\mathrm{Fe} / \mathrm{H}]_{\mathrm{cor}}(\mathrm{E} 00)$} & & $-1.63(0.10)$ & & $-1.23(0.12)$ & & $-0.83(0.14)$ \\
\hline & {$[\mathrm{Fe} / \mathrm{H}]_{\mathrm{cor}}(\mathrm{E} 11)$} & & $-1.63(0.10)$ & & $-1.15(0.13)$ & & $-0.83(0.14)$ \\
\hline & {$[\mathrm{Fe} / \mathrm{H}]_{\text {cor }}(\mathrm{S} 00)$} & & $-1.52(0.10)$ & & $-0.95(0.13)$ & & $-0.69(0.14)$ \\
\hline & {$[\mathrm{Fe} / \mathrm{H}]_{\text {cor }}(\mathrm{S} 11)$} & & $-1.52(0.10)$ & & $-0.95(0.13)$ & & $-0.69(0.14)$ \\
\hline $\log N(\mathrm{Cr} I \mathrm{I})$ & {$[\mathrm{Cr} / \mathrm{H}]_{\mathrm{obs}}$} & $13.37(0.01)$ & $-1.69(0.08)$ & $12.95(0.03)$ & $-1.88(0.09)$ & $13.00(0.04)$ & $-1.22(0.09)$ \\
\hline & {$[\mathrm{Cr} / \mathrm{H}]_{\text {cor }}(\mathrm{E} 00)$} & & $-1.61(0.09)$ & & $-1.23(0.13)$ & & $-0.94(0.13)$ \\
\hline & {$[\mathrm{Cr} / \mathrm{H}]_{\text {cor }}(\mathrm{E} 11)$} & & $-1.60(0.10)$ & & $-1.14(0.15)$ & & $-0.94(0.13)$ \\
\hline & {$[\mathrm{Cr} / \mathrm{H}]_{\text {cor }}(\mathrm{S} 00)$} & & $-1.53(0.10)$ & & $-1.07(0.12)$ & & $-0.80(0.13)$ \\
\hline & {$[\mathrm{Cr} / \mathrm{H}]_{\text {cor }}(\mathrm{S} 11)$} & & $-1.51(0.10)$ & & $-0.94(0.13)$ & & $-0.82(0.13)$ \\
\hline $\log N(\mathrm{Ni}$ II $)$ & {$[\mathrm{Ni} / \mathrm{H}]_{\mathrm{obs}}$} & $13.87(0.01)^{a}$ & $-1.75(0.08)^{a}$ & $13.44(0.08)$ & $-1.95(0.11)$ & $13.54(0.06)$ & $-1.24(0.10)$ \\
\hline & {$[\mathrm{Ni} / \mathrm{H}]_{\text {cor }}(\mathrm{E} 00)$} & & $-1.62(0.10)$ & & $-1.23(0.15)$ & & $-0.91(0.14)$ \\
\hline & {$[\mathrm{Ni} / \mathrm{H}]_{\mathrm{cor}}(\mathrm{E} 11)$} & & $-1.60(0.10)$ & & $-1.09(0.17)$ & & $-0.87(0.15)$ \\
\hline & {$[\mathrm{Ni} / \mathrm{H}]_{\text {cor }}(\mathrm{S} 00)$} & & $-1.52(0.10)$ & & $-0.95(0.14)$ & & $-0.78(0.14)$ \\
\hline & {$[\mathrm{Ni} / \mathrm{H}]_{\text {cor }}(\mathrm{S} 11)$} & & $-1.50(0.10)$ & & $-0.88(0.15)$ & & $-0.73(0.14)$ \\
\hline $\log N(\mathrm{Mn}$ II $)$ & {$[\mathrm{Mn} / \mathrm{H}]_{\mathrm{obs}}^{\dagger}$} & $\ldots$ & $\ldots$ & $12.50(0.03)$ & $-2.17(0.09)$ & $12.59(0.04)$ & $-1.47(0.09)$ \\
\hline $\log N(\mathrm{Si}$ II $)$ & {$[\mathrm{Si} / \mathrm{H}]_{\mathrm{obs}}$} & $>14.72^{a}$ & $>-2.21^{a}$ & $15.30(0.01)$ & $-1.40(0.08)$ & $15.29(0.04)$ & $-0.80(0.09)$ \\
\hline & {$[\mathrm{Si} / \mathrm{H}]_{\text {cor }}(\mathrm{E} 00)$} & & $>-2.21$ & & $-1.22(0.11)$ & & $-0.78(0.10)$ \\
\hline & {$[\mathrm{Si} / \mathrm{H}]_{\text {cor }}(\mathrm{E} 11)$} & & $>-2.21$ & & $-1.16(0.12)$ & & $-0.78(0.10)$ \\
\hline & {$[\mathrm{Si} / \mathrm{H}]_{\text {cor }}(\mathrm{S} 00)$} & & $>-2.21$ & & $-0.98(0.11)$ & & $-0.74(0.11)$ \\
\hline & {$[\mathrm{Si} / \mathrm{H}]_{\text {cor }}(\mathrm{S} 11)$} & & $>-2.21$ & & $-1.01(0.11)$ & & $-0.74(0.10)$ \\
\hline $\log N(\mathrm{~S}$ II $)$ & {$[\mathrm{S} / \mathrm{H}]_{\mathrm{obs}}$} & $15.09(0.06)$ & $-1.48(0.11)$ & $15.08(0.11)$ & $-1.26(0.14)$ & $15.10(0.15)$ & $-0.63(0.17)$ \\
\hline $\log N(\mathrm{Mg}$ II $)$ & {$[\mathrm{Mg} / \mathrm{H}]_{\mathrm{obs}}$} & $15.57(0.09)$ & $-1.38(0.11)$ & $15.53(0.14)$ & $-1.19(0.15)$ & $\ldots$ & $\ldots$ \\
\hline & {$[\mathrm{Mg} / \mathrm{H}]_{\text {cor }}(\mathrm{E} 00)$} & & $-1.38(0.11)$ & & $-1.05(0.16)$ & & $\ldots$ \\
\hline & {$[\mathrm{Mg} / \mathrm{H}]_{\text {cor }}(\mathrm{E} 11)$} & & $-1.38(0.11)$ & & $-0.90(0.19)$ & & $\ldots$ \\
\hline & {$[\mathrm{Mg} / \mathrm{H}]_{\text {cor }}(\mathrm{S} 00)$} & & $-1.37(0.11)$ & & $-0.87(0.17)$ & & $\ldots$ \\
\hline & {$[\mathrm{Mg} / \mathrm{H}]_{\text {cor }}(\mathrm{S} 11)$} & & $-1.37(0.11)$ & & $-0.76(0.18)$ & & $\ldots$ \\
\hline $\log N(\mathrm{Ti}$ II $)$ & {$[\mathrm{Ti} / \mathrm{H}]_{\mathrm{obs}}{ }^{*}$} & $<12.21^{b}$ & $<-2.10^{b}$ & $<11.34$ & $<-2.74$ & $12.66(0.08)$ & $-0.81(0.11)$ \\
\hline $\log N(\operatorname{Ar} \mathrm{I})$ & {$[\mathrm{Ar} / \mathrm{H}]_{\mathrm{obs}}$} & $14.21(0.12)$ & $-1.68(0.15)$ & $\ldots$ & $\ldots$ & $\ldots$ & $\ldots$ \\
\hline $\log N(\mathrm{~N} \mathrm{I})$ & {$[\mathrm{N} / \mathrm{H}]_{\mathrm{obs}}$} & $15.03(0.10)$ & $-2.31(0.14)$ & $<15.23$ & $<-1.88$ & $<15.02$ & $<-1.48$ \\
\hline $\log N(\mathrm{P}$ II $)$ & {$[\mathrm{P} / \mathrm{H}]_{\mathrm{obs}}$} & $13.05(0.09)$ & $-1.85(0.13)$ & $13.25(0.10)$ & $-1.42(0.13)$ & $<13.51$ & $<-0.55$ \\
\hline $\log N(\mathrm{Al}$ II $)$ & {$[\mathrm{Al} / \mathrm{H}]_{\mathrm{obs}}$} & ... & $\ldots$ & $>13.74^{a}$ & $>-1.89^{a}$ & $\ldots$ & $\ldots$ \\
\hline $\log N(\mathrm{Cl} \mathrm{I})$ & {$[\mathrm{Cl} / \mathrm{H}]_{\mathrm{obs}}$} & $\ldots$ & $\ldots$ & $13.05(0.10)$ & $>-1.37^{c}$ & $\ldots$ & $\ldots$ \\
\hline
\end{tabular}

${ }^{a}$ Prochaska \& Wolfe (1999).

${ }^{b}$ Prochaska et al. (2001).

${ }^{c}$ The $[\mathrm{Cl} / \mathrm{H}]$ absolute abundance derived from the $\mathrm{Cl}$ I column density has to be considered as a strict lower limit, since the dominant state of $\mathrm{Cl}$ should be $\mathrm{Cl}$ II in DLAs.

$\dagger$ We do not compute the dust corrections for $[\mathrm{Mn} / \mathrm{H}]$, since this element when analyzed is compared with Fe, and these two elements have very similar dust depletions.

* We do not compute the dust corrections for [Ti/H], since this element when analyzed is compared with Fe, and in this case dust depletion and nucleosynthesis tend to work in the opposite sense (see Paper I).

In the dust correction models E00 and E11, Vladilo (2002a) assumes that the intrinsic [Zn/Fe] ratio is equal to +0.10 dex.

In the dust correction models S00 and S11, Vladilo (2002a) assumes that the intrinsic $[\mathrm{Zn} / \mathrm{Fe}]$ ratio is equal to $+0.00 \mathrm{dex}$.

total Fe abundance generally derived from strong Fe II lines. In the three DLAs studied this effect is particularly important in the DLA toward Q2231-00 which show complex metal-line profiles, with a large number of components. The same approach has already been used in Paper I.

\subsection{Intrinsic abundance patterns}

In this section we describe the derived intrinsic abundance patterns of the DLAs toward Q0100+13, Q1331+17 and Q2231-00. Their interpretation will be presented in Sect. 6. To present the abundance patterns we use the same type of diagrams as the ones used by Prochaska et al. (2003). The dustcorrected elemental abundances are plotted on a logarithmic scale, where hydrogen is defined to have $\epsilon(\mathrm{H})=12$ and, in a general way, for any element $\mathrm{X}, \epsilon(\mathrm{X})=\log (\mathrm{X} / \mathrm{H})+12$, and are compared with the solar abundance pattern from Grevesse \& Sauval (1998) (shown by the solid line) scaled to match the observed sulfur metallicity of each DLA system. This way of showing the elemental abundances as a function of the atomic number, $Z$, has the advantage that we see in the same plot all the measured abundances in a system, and this allows us to directly identify possible deviations from solar values. 
Table 9. Summary of the relative abundances in the three DLA systems studied.

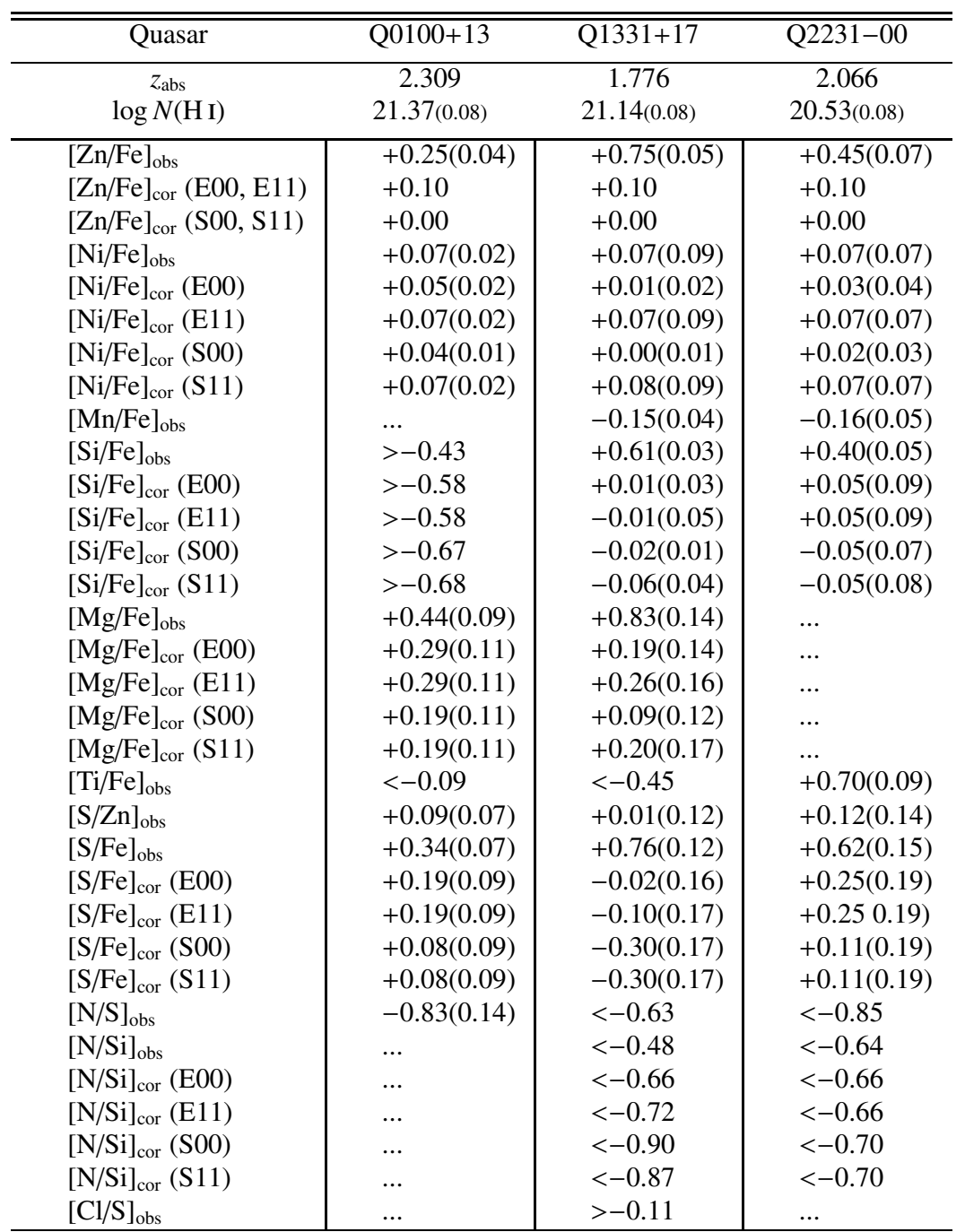

Figures 9-11 show the nucleosynthetic patterns of the DLAs toward Q0100+13, Q1331+17 and Q2231-00, respectively. At a first glance, the abundance patterns of these high redshift galaxies resemble that of the solar neighborhood indicating that their nucleosynthetic enrichment histories are not too dissimilar from our Galaxy. However, at closer inspection, one notes several important differences.

\subsection{1. $\mathrm{Q} 0100+13, z_{\mathrm{abs}}=2.309$}

The DLA toward Q0100+13 (Fig. 9) shows a slight enhancement of the $\alpha$-elements $\mathrm{S}$ and $\mathrm{Mg}$ abundances relative to the $\mathrm{Zn}$ and Fe-peak element abundances, namely $[\mathrm{S} / \mathrm{Zn}]=+0.19 \pm$ 0.09 and $[\mathrm{Mg} / \mathrm{Fe}]_{\text {cor }}=+0.29 \pm 0.11$. This $\alpha / \mathrm{Fe}$-peak enhancement is suggestive of an enrichment by massive stars. Indeed, the $\alpha$-elements are produced in less than $2 \times 10^{7} \mathrm{yrs}$ by type II supernovae $(\mathrm{SNe})$ resulting from massive stars and the $\mathrm{Fe}-$ peak elements are mainly produced by type Ia $\mathrm{SNe}$ on longer timescales, between $10^{8}-10^{9} \mathrm{yrs}$.

The dust-corrected ratio of the two $\alpha$-elements $\mathrm{Mg}$ and $\mathrm{S},[\mathrm{Mg} / \mathrm{S}]_{\text {cor }}=+0.10 \pm 0.12$, shows that these two elements closely track each other in the DLA. On the other hand, the $\alpha$-element $\mathrm{Ar}$ is slightly underabundant relative to $\mathrm{S}$, as noted in Sect. 4. The Fe-peak elements $\mathrm{Fe}, \mathrm{Ni}$ and $\mathrm{Cr}$ show solar values one relative to the other, as observed in the Galactic stars with similar metallicities (see Table 8).

In this system, one also observes the odd-even effect, which corresponds to an underabundance of odd- $Z$ elements relative to the even- $Z$ elements of the same nucleosynthetic origin. Indeed, we obtained $[\mathrm{P} / \mathrm{S}]=-0.37 \pm 0.12$ at $[\mathrm{P} / \mathrm{H}]=$ $-1.85 \pm 0.13$, which even shows evidence for an enhanced odd-even effect. This value is similar to the $[\mathrm{P} / \mathrm{Si}]=-0.21$ at $[\mathrm{P} / \mathrm{H}]=-1.16,[\mathrm{P} / \mathrm{Si}]=-0.40$ at $[\mathrm{P} / \mathrm{H}]=-2.30$ and $[\mathrm{P} / \mathrm{Si}]=-0.30$ at $[\mathrm{P} / \mathrm{H}]=-1.20$ values measured toward other DLAs by Levshakov et al. (2002), Molaro et al. (2001) and Outram et al. (1999), respectively, and is indicative of massive supernovae. Phosphorus abundance measurements in DLAs are very important to understand the nucleosynthesis of this element, because no $\mathrm{P}$ abundance measurement exists in the Galactic stars. The observed odd-even effect in the $\mathrm{P} / \mathrm{S}$ ratio is in agreement with the findings of Goswami \& Prantzos (2000). 


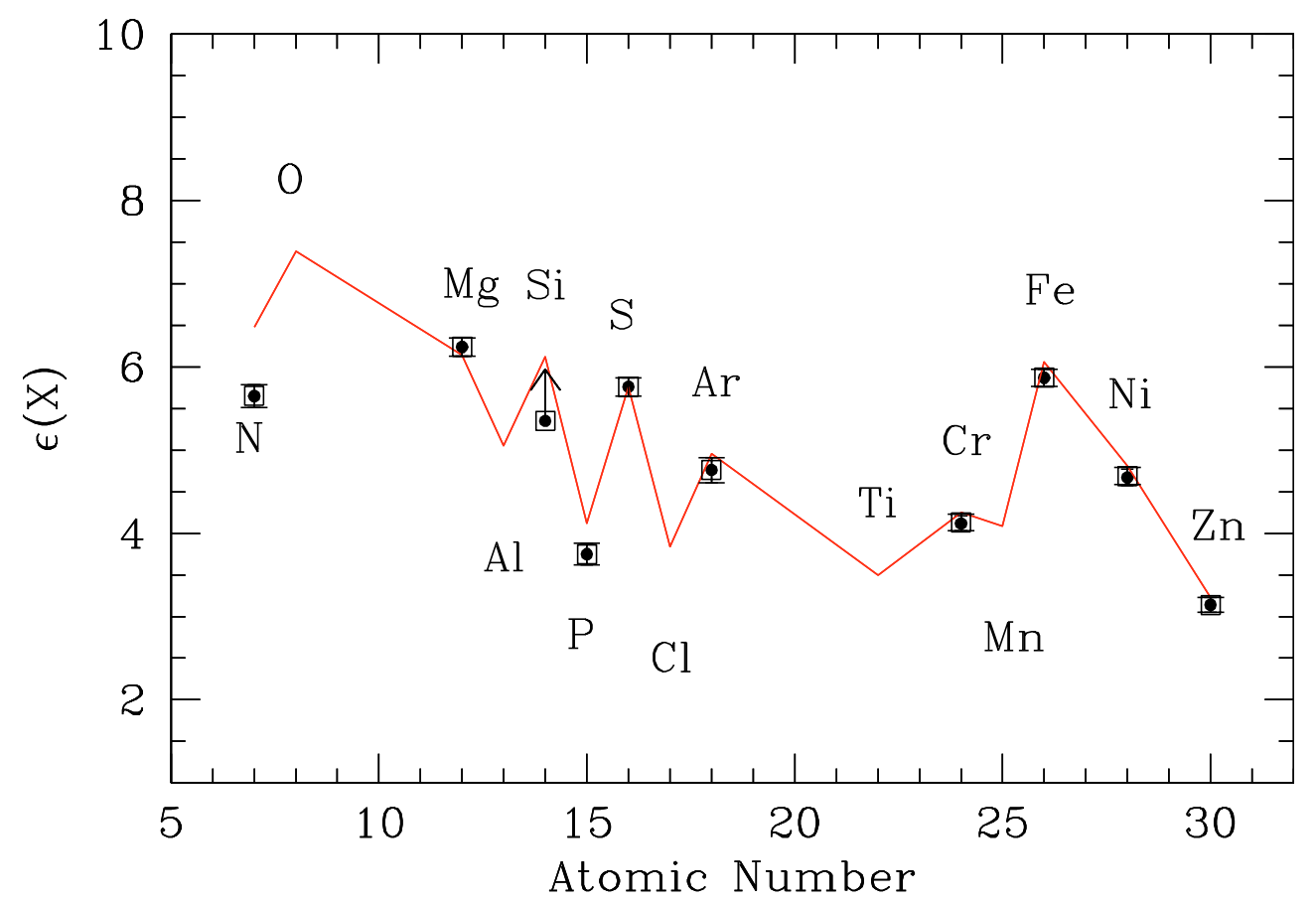

Fig. 9. The nucleosynthetic abundance pattern of the DLA at $z_{\text {abs }}=2.309$ toward Q0100+13. The dust-corrected elemental abundances are represented on a logarithmic scale, where hydrogen is defined to have $\epsilon(\mathrm{H})=12$ and in a general way, for any element $\mathrm{X}, \epsilon(\mathrm{X})=\log (\mathrm{X} / \mathrm{H})+12$. They are compared with the solar abundance pattern from Grevesse \& Sauval (1998) shown by the solid line and scaled to match the observed sulfur metallicity, $[\mathrm{S} / \mathrm{H}]=-1.44$, of the DLA system. The dust corrections were obtained via the Vladilo (2002a,b) method. The filled circles correspond to the dust-corrected abundances obtained with the E00 dust correction model and the open squares to the dust-corrected abundances obtained with the E11 dust correction model.

Finally, the DLA shows an undersolar $[\mathrm{N} / \mathrm{S}]$ ratio of $-0.83 \pm 0.14$ at $[\mathrm{S} / \mathrm{H}]=-1.48 \pm 0.11 . \mathrm{N}$ is an element of particular interest, since it has a complex nucleosynthetic origin. Synthesized in the $\mathrm{CNO}$ cycle of stars, $\mathrm{N}$ is either a secondary element in the sense that it is produced in proportion to $\mathrm{C}$ and $\mathrm{O}$ originally present in the star, or a primary element in the sense that it can be produced starting from $\mathrm{C}$ and $\mathrm{O}$ manufactured by the star "in situ". In the $\mathrm{N} / \alpha$ versus $\alpha / \mathrm{H}$ diagram, the secondary $\mathrm{N}$ over $\alpha$-element ratio is expected to increase steeply with the increasing metallicity, while the primary $\mathrm{N}$ over $\alpha$ element ratio remains constant when the metallicity increases (Talbot \& Arnett 1974). The progenitors of primary N have not been fully constrained yet. The most recent stellar models of Meynet \& Maeder (2002) including the stellar rotation indicate that the intermediate-mass stars are the main producers of $\mathrm{N}$. The $[\mathrm{N} / \mathrm{S}]$ ratio reached in the DLA toward Q0100+13 is a "high" value, in the sense that it is close to the primary $\mathrm{N}$ "plateau" at $[\mathrm{N} / \mathrm{S}] \simeq-0.75$. It is at the upper end of the range of $\mathrm{N} / \mathrm{S}$ values measured in DLAs and in good agreement with the typical values observed in $\mathrm{H}$ II regions at similar metallicities (e.g. Izotov \& Thuan 1999; Pilyugin et al. 2002).

\subsection{2. $\mathrm{Q} 1331+17, z_{\mathrm{abs}}=1.776$}

The DLA toward Q1331+17 (Fig. 10) has almost a solar abundance pattern. Very few differences are observed. Indeed, the Fe-peak elements $\mathrm{Fe}, \mathrm{Ni}$ and $\mathrm{Cr}$ closely track each other, the two $\alpha$-elements $\mathrm{S}$ and $\mathrm{Si}$ also (see Table 8), and the $\alpha$-element over Fe-peak element ratios - Si/Fe, S/Zn and S/Fe - are all solar. Only the $\mathrm{Mg} / \mathrm{Fe}$ ratio is slightly oversolar (see Table 9). We would like to note that the S00 and S11 dust correction models of Vladilo (2002a) which assume a solar ratio of $\mathrm{Zn} / \mathrm{Fe}$, lead to $[\mathrm{S} / \mathrm{Fe}]_{\text {cor }}=-0.30 \pm 0.17$ (see Table 9). Such an undersolar $\alpha$-element over Fe-peak element ratio is neither observed in Galactic stars nor predicted by chemical evolution models. Thus, this provides evidence that $[\mathrm{Zn} / \mathrm{Fe}]>0$ (intrinsically) in this DLA system. Ti exhibits a large underabundance relative to the Fe-peak elements and this, although Ti is an $\alpha$-element, and even after having applied the dust corrections. At a first glance, the only way to explain this observation is that we have underestimated the Ti upper limit.

We measure the abundances of $\mathrm{P}$ and $\mathrm{Mn}$, two odd- $\mathrm{Z}$ elements. The odd-even effect is less marked in this system than in the DLA toward Q0100+13. We get $[\mathrm{P} / \mathrm{Si}]_{\mathrm{cor}}=-0.20 \pm 0.11$ when the $\mathrm{E} 00$ dust correction is applied and $[\mathrm{P} / \mathrm{Si}]_{\text {cor }}=-0.26 \pm$ 0.12 when the $\mathrm{E} 11$ dust correction is applied at $[\mathrm{P} / \mathrm{H}]=-1.42 \pm$ 0.13 , and $[\mathrm{Mn} / \mathrm{Fe}]=-0.15 \pm 0.04$ (without dust corrections, and $-0.07 \pm 0.05,-0.37 \pm 0.05$ when the E00, E11 dust corrections are applied, respectively) at $[\mathrm{Fe} / \mathrm{H}]_{\mathrm{cor}}=-1.23 \pm 0.12$.

We have detected the ClI $\lambda 1347$ line in this DLA system, which allows us to measure the $\mathrm{Cl}^{0}$ column density and to provide a lower limit on the total $\mathrm{Cl}$ absolute abundance. $\mathrm{Cl}$ is also an odd- $\mathrm{Z}$ element. This is the third $\mathrm{Cl}$ abundance upper limit derived in a DLA system, the other two were obtained by Ledoux et al. (2002) and Prochaska et al. (2003). Cl abundance measurements in DLAs are very important to constrain the $\mathrm{Cl}$ nucleosynthesis in the low metallicity regime, in particular because no $\mathrm{Cl}$ abundance measurement exists in Galactic stars. 


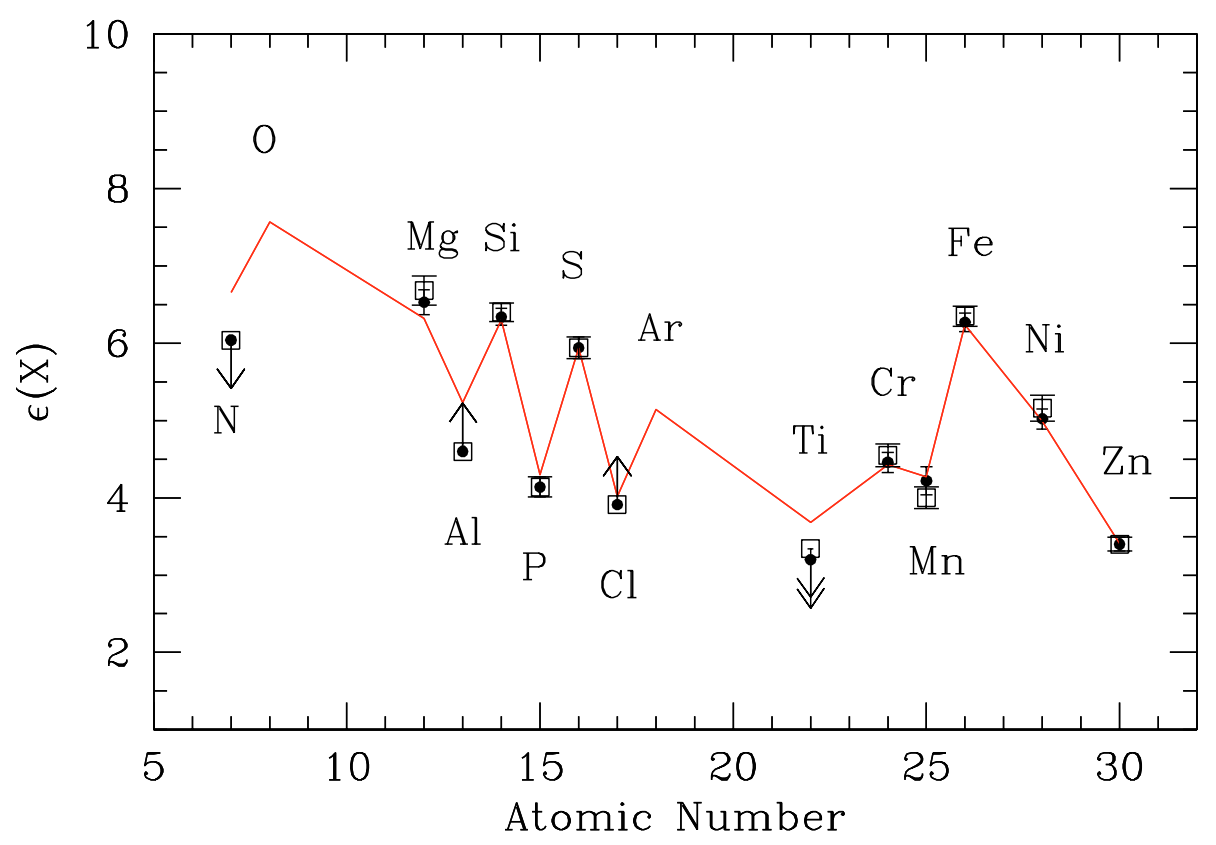

Fig. 10. The nucleosynthetic abundance pattern of the DLA at $z_{\mathrm{abs}}=1.776$ toward Q1331+17. The solar values were scaled to match the observed sulfur metallicity, $[\mathrm{S} / \mathrm{H}]=-1.26$. Same as Fig. 9 .

$\mathrm{Cl}$ measurements are available in planetary nebulae of the Galactic disk (PNe; e.g. Costa et al. 1996; Kwitter et al. 2003) and in the Galactic disk ISM (e.g. Welty et al. 1999). Oversolar $[\mathrm{Cl} / \mathrm{S}]$ ratios between +0.09 and $+0.5 \mathrm{dex}$ are observed in type II PNe, and undersolar $[\mathrm{Cl} / \mathrm{S}]$ ratios of -0.6 and -0.2 dex are measured in the cold and warm Galactic disk ISM, respetively, suggesting that $\mathrm{Cl}$ is dust depleted. In the DLA studied here we derive a lower limit on the $[\mathrm{Cl} / \mathrm{S}]$ ratio of $>-0.11$ at $[\mathrm{Cl} / \mathrm{H}]>-1.37$, and in the DLA studied by Ledoux et al. (2002) and Prochaska et al. (2003) $[\mathrm{Cl} / \mathrm{S}]>-0.33$ and $>-0.68$, respectively. These three lower limits measured in DLAs suggest that the $\mathrm{Cl} / \mathrm{S}$ ratios likely are solar to oversolar similarly to what is observed in $\mathrm{PNe}$, thus leaving very little space for an odd-even effect for $\mathrm{Cl}$. The chemical evolution models of Goswami \& Prantzos (2000) computed with the Woosley \& Weaver (1995) yields predict a solar $\mathrm{Cl} / \mathrm{S}$ ratio, and hence a negligible odd-even effect for $\mathrm{Cl}$.

Prochaska \& Wolfe (1999) also reported the detection of $\mathrm{C} \mathrm{II}^{*}$ in the DLA toward Q1331+17. The presence of both strong $\mathrm{C} \mathrm{II}^{*}$ and $\mathrm{Cl} \mathrm{I}$ absorptions suggests that the gas resides in a cold neutral medium, characteristic of highly depleted gas in the Milky Way. This DLA precisely shows an important dust depletion level, with $[\mathrm{Zn} / \mathrm{Fe}]=+0.75 \pm 0.05$. Furthermore, the observation of significant $\mathrm{Cl}$ I requires at least a modest molecular hydrogen fraction. Indeed, in regions where $\mathrm{H}_{2}$ is optically thick, $\mathrm{Cl}$ II reacts rapidly with $\mathrm{H}_{2}$ to form $\mathrm{HCl}^{+}$, which in turn leads to $\mathrm{Cl}$ I and $\mathrm{HI}$. Because the conversion of $\mathrm{Cl}$ II to $\mathrm{Cl}$ I is faster than the photoionization of $\mathrm{Cl} \mathrm{I}, \mathrm{Cl}$ is primarily neutral in regions where $\mathrm{H}_{2}$ is abundant but otherwise is primarily ionized (Jura \& York 1978).

Finally, the derived upper limits on $[\mathrm{N} / \mathrm{S}]$ and $[\mathrm{N} / \mathrm{Si}]$ are at the upper end of the range of $\mathrm{N} / \alpha$ values measured in DLAs (see Table 9). They are close to the primary N "plateau".

\subsection{3. $Q 2231-00, z_{\mathrm{abs}}=2.066$}

The DLA toward Q2231-00 (Fig. 11) shows a solar [Si/Fe] ratio, but enhanced $[\mathrm{S} / \mathrm{Zn}, \mathrm{Fe}]$ and $[\mathrm{Ti} / \mathrm{Zn}, \mathrm{Fe}]$ ratios (see Table 9). The $\mathrm{S}$ abundance was derived from a single $\mathrm{S}$ II line located in the $\operatorname{Ly} \alpha$ forest (see Sect. 3.3), and we believe that it may be partly blended with H I clouds. The comparison of the dustcorrected absolute abundances of $\mathrm{Si}$ and $\mathrm{S}$, two $\alpha$-elements, shows further evidence for a slight overestimation of the abundance of $\mathrm{S}$, since $[\mathrm{S} / \mathrm{Si}]_{\text {cor }}=+0.19 \pm 0.16$ when the E00 dust correction is applied. Although the adopted large error on the $\mathrm{S}$ abundance measurement takes into account this slight overestimation, in Fig. 11 we scale the solar values to match the dust corrected silicon metallicity, instead of the observed sulfur metallicity as done in Figs. 9 and 10. The Ti abundance is also very difficult to measure, thus it is very likely that it is overestimated, as already suspected in Sect. 5.1.

The Fe-peak elements, $\mathrm{Fe}, \mathrm{Ni}$ and $\mathrm{Cr}$, track very well each other, as observed in the Galactic stars with similar metallicities (see Table 8). With the abundance measurement of the Fe-peak element $\mathrm{Mn}$, we highlight a rather pronounced odd-even effect in this DLA system, $[\mathrm{Mn} / \mathrm{Fe}]=-0.16 \pm 0.05$ at $[\mathrm{Fe} / \mathrm{H}]_{\text {cor }}=$ $-0.83 \pm 0.14$. Another odd-Z element detected in this system is $\mathrm{P}$. Only an upper limit on the $[\mathrm{P} / \mathrm{Si}]$ abundance ratio is derived, $[\mathrm{P} / \mathrm{Si}]_{\text {cor }}<+0.34$, which is potentially in line with an odd-even effect for $\mathrm{P}$.

The obtained [N/Si, S] upper limits (see Table 9) are again "high", close to the primary N "plateau". We do not believe that the observed N I lines are heavily blended with Ly $\alpha$ forest lines. 


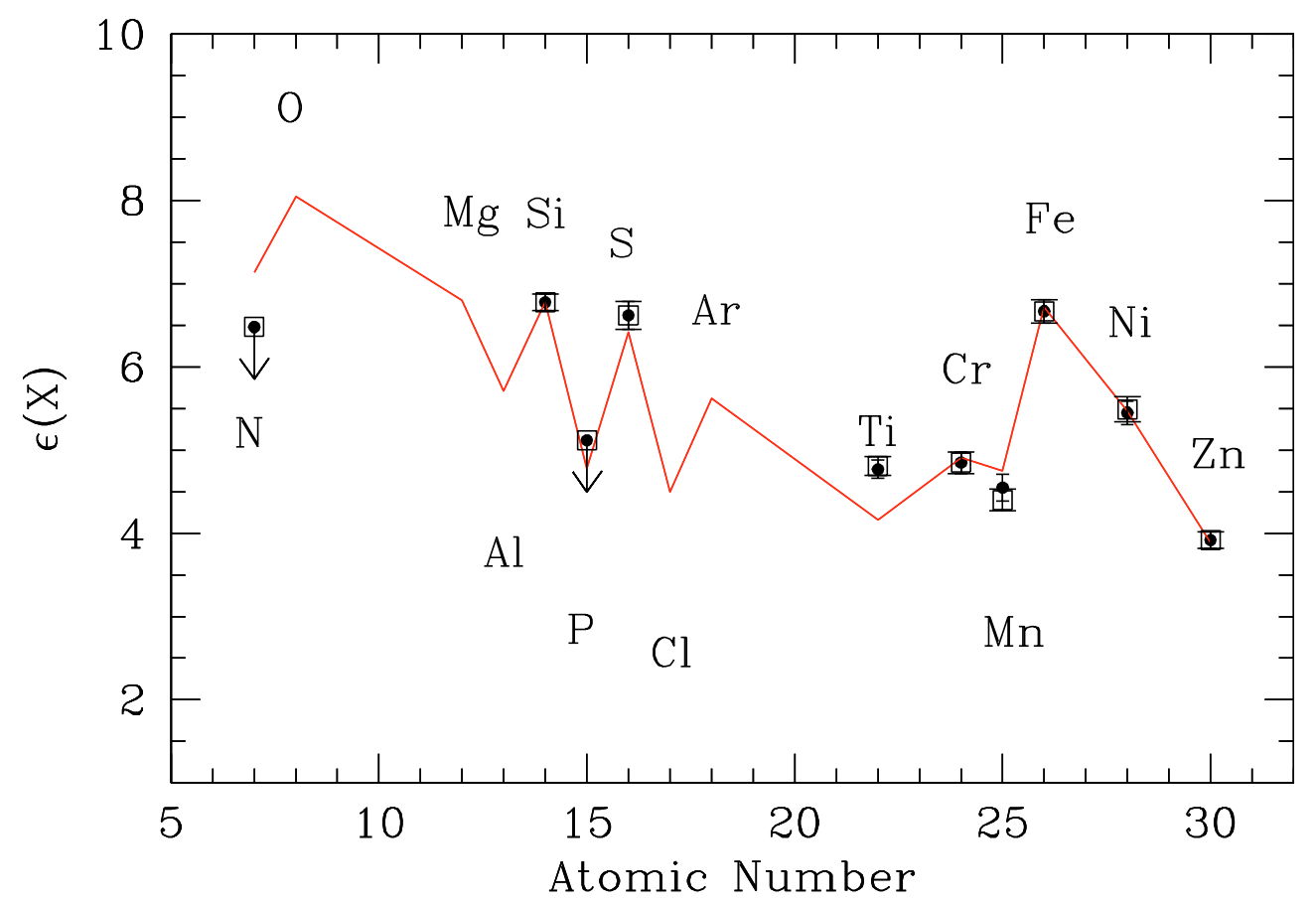

Fig. 11. The nucleosynthetic abundance pattern of the DLA at $z_{\mathrm{abs}}=2.066$ toward Q2231-00. The solar values were scaled to match the dust-corrected silicon metallicity, $[\mathrm{Si} / \mathrm{H}]_{\mathrm{cor}}=-0.78$. Same as Fig. 9.

\section{Determination of the star formation histories}

Until now the DLA galaxy population has been analyzed as a whole. Several chemical evolution models were constructed in order to interpret the abundance patterns observed in DLAs as an ensemble, considering them as an evolutionary sequence, i.e. objects caught in a different phase of their evolution (e.g. Matteucci et al. 1997; Jimenez et al. 1999; Hou et al. 2001; Mathlin et al. 2001). However, several pieces of evidence - like the low redshift deep imaging revealing a variety of morphological types belonging to the DLA population (e.g. Le Brun et al. 1997; Nestor et al. 2002), the large scatter in the $\alpha$ over Fe-peak element abundance ratios at a given metallicity and the large scatter observed in the metallicities - indicate that the DLAs trace galaxies with different star formation histories. Some may have formed stars on timescales similar to that of the early Milky Way, while others apparently did so more slowly or intermittently, so that the Fe-peak elements could catch up with the $\alpha$-elements.

Thanks to the large number of elements detected in the DLA systems studied in this paper, we are for the first time in a very appropriate situation to analyze these high redshift galaxies individually. The different elemental abundances at disposal allow us, in particular, to constrain the star formation history (hereafter SFH, and SF for the star formation) and the age of the associated galaxies by means of a direct comparison with a grid of chemical evolution models for spiral and dwarf irregular galaxies (see Calura et al. 2003) rather than just by means of a comparison with the solar abundance pattern as presented in Sect. 5.3. We present below the approach which has been applied. The derived results for the DLAs toward Q0100+13, Q1331+17 and Q2231-00 are summarized in Table 10.

\subsection{Chemical evolution models}

A chemical evolution model allows one to follow in detail the evolution of abundances of several chemical species, starting from the matter reprocessed by the stars and restored into the ISM through stellar winds and supernova explosions. Here we briefly summarize the major ingredients of the chemical evolutions models used in this work which we identify as "spiral" and "dwarf irregular" models according to the type of galaxies they do match best. These models are the same as the models used by Calura et al. (2003) in their study of the DLA nature. A detailed description of the "spiral" model can be found in Chiappini et al. $(1997,2001)$ and of the "dwarf irregular" model in Bradamante et al. (1998) and Recchi et al. (2001, 2002). In both models no instantaneous recycling approximation is adopted, namely the stellar lifetimes are properly taken into account.

\subsection{1. "Spiral" model}

The spiral galaxies are assumed to form as a result of two main infall episodes (Chiappini et al. 1997). During the first episode the halo forms and the gas shed by the halo rapidly gathers in the center leading to the formation of the bulge. During the second episode, a slower infall of external gas gives rise to the disk with the gas accumulating faster in the inner than in the outer region ("inside-out" scenario, Matteucci \& François 1989). The process of disk formation is much longer than the halo and bulge formation, with timescales varying from $\sim 2 \mathrm{Gyr}$ in the inner disk to $\sim 7 \mathrm{Gyr}$ in the solar region and up to 13 Gyr in the outer disk. In particular, a timescale of $7 \mathrm{Gyr}$ in the solar neighborhood is required to fit the G-dwarf metallicity 
Table 10. Nature of the galaxies associated with the DLA systems studied.

\begin{tabular}{lcccc}
\hline \hline DLA system & Model & $\begin{array}{c}\text { Star formation characteristics } \\
v\left[\mathrm{Gyr}^{-1}\right] / \Delta t[\mathrm{Gyr}] / t_{\mathrm{b}}[\mathrm{Gyr}]\end{array}$ & $z_{\mathrm{f}}$ & $\begin{array}{c}\text { Age } \\
{[\mathrm{Gyr}]}\end{array}$ \\
\hline Q0100+13, $z_{\text {abs }}=2.309$ & Spiral & solar neighborhood: $R=8 \mathrm{kpc}$ & $\sim 2.5$ & $0.25 \pm 0.20$ \\
& Dwarf irregular & bursting SF: $0.9 / 0.07 / 2.0$ & $(2.35-2.47)$ & $0.05-0.20$ \\
Q1331+17, $z_{\text {abs }}=1.776$ & Spiral & outer disk: $R=16 \mathrm{kpc}$ & $\sim 2.8$ & $1.5 \pm 0.4$ \\
& Dwarf irregular & continuous SF: $0.03 / 13 / \ldots$ & $\sim 10$ & $\gtrsim 3.5$ \\
Q2231-00, $z_{\text {abs }}=2.066$ & Dwarf irregular & bursting SF: $4.2 / 0.10 / 0.5$ & $(2.10-2.16)$ & $0.05-0.15$ \\
\hline
\end{tabular}

$z_{\mathrm{f}}$ in brackets $=$ Values obtained under the assumption that the galaxy has undergone a single burst of SF.

distribution (Chiappini et al. 1997; Boissier \& Prantzos 1999). The adopted $S F R$ expression is:

$\psi(R, t)=v\left[\frac{\sigma(R, t)}{\sigma\left(R_{\odot}, t\right)}\right]^{2(k-1)}\left[\frac{\sigma\left(R, t_{\mathrm{Gal}}\right)}{\sigma(R, t)}\right]^{k-1} \sigma_{\mathrm{gas}}^{k}(R, t)$

where $v$ is the star formation efficiency, namely the inverse of the typical timescale for star formation, $\sigma(R, t)$ is the total (gas + stars) mass surface density at a radius $R$ and time $t$, $\sigma\left(R_{\odot}, t\right)$ is the total mass surface density in the solar region, and $\sigma_{\text {gas }}(R, t)$ is the gas mass surface density. For the gas density exponent $k$ a value of 1.5 has been assumed by Chiappini et al. (1997) in order to ensure a good fit to the observational constraints in the solar vicinity. The star formation efficiency is set to $v=1 \mathrm{Gyr}^{-1}$, and becomes zero when the gas surface density drops below a certain critical threshold. A threshold density $\sigma_{\text {th }} \sim 7 M_{\odot} \mathrm{pc}^{-2}$ is adopted in the disk as suggested by Chiappini et al. (1997). The initial mass function (IMF) is taken from Scalo (1986). The galactic wind is considered not efficient (Matteucci 2001). All the input parameters of this "spiral" model were constrained in order to successfully reproduce the observed properties first in the solar vicinity, such as the solar abundances, the G-dwarf metallicity distribution (see above) and the abundance ratios, and then at the global scale, such as the radial gas and stellar density profiles and the abundance gradients of various elements observed in the stars of the Milky Way.

Due to the "inside-out" scenario and the threshold density adopted in the "spiral" model, the SFH is different at different galactocentric radii. Indeed, in the inner regions of the disk where the rate of accretion of matter onto the disk is fast, a high gas surface density, well above the critical threshold, is reached and maintained during a long period, and hence the SF is almost continuous through the galaxy lifetime. On the other hand, in the outer regions of the disk (radius $R \gtrsim 12 \mathrm{kpc}$ ) where the rate of accretion of matter onto the disk is slow, the SFH proceeds in a gasping way, due to the fact that in these regions the gas is always close to the critical threshold. As a consequence, the external regions of the disk look like the Magellanic irregulars where this kind of SF seems to take place (Tosi et al. 1991). These different star formation histories at different galactocentric radii are associated with different abundance patterns which can be compared with observations.

When comparing the "spiral" model with our observations of DLA systems, we run a set of models each of them corresponding to a different galactocentric radius $R$. This radius represents the position at which the QSO line of sight crosses the disk of the observed DLA galaxy. Finally, we need to constrain the formation redshift, $z_{\mathrm{f}}$, of the "spiral" model, which is the single free parameter.

\subsection{2. "Dwarf irregular" model}

We adopt a model based on the work of Bradamante et al. (1998), in which the dwarf irregular galaxies form owing to a continuous infall of pristine gas with an infall timescale of $0.5 \mathrm{Gyr}$, until a mass of $\sim 10^{9} M_{\odot}$ is accumulated. The evolution of dwarf irregular galaxies is assumed to be characterized by $a$ bursting star formation history. This particular model was built to reproduce the chemical properties of the local blue compact galaxies (BCGs). The parameters which need to be defined in this model are the number of bursts of SF that the galaxy has undergone, and for each burst, the star formation efficiency, $v$, (same definition as in the "spiral" model), the burst duration, $\Delta t$, and the time of occurrence of the burst, $t_{\mathrm{b}}$, i.e. the time necessary for the infall of pristine gas before the SF starts. Hence, the star formation rate in the "dwarf irregular" model can proceed either in short bursts of a duration from 10 to $200 \mathrm{Myr}$ separated by long quiescent periods, or at a low regime but continuously, namely in one or two long episodes of inefficient SF lasting between 3 and 13 Gyr. In this model, the adopted $S F R$ is:

$\psi(t)=v \sigma_{\mathrm{gas}}(t)$

where $\sigma_{\text {gas }}(t)$ represents the gas density at time $t$ and $v$ is the star formation efficiency.

The dwarf irregular galaxies are particularly sensitive to outflows resulting from the energy injection from the SN explosions. Indeed, galactic winds develop when the thermal energy of the gas equals its binding energy (Matteucci \& Tornambé 1987). In the "dwarf irregular" model used in this paper, the rate of gas loss via galactic winds is assumed to be proportional to the SF, and the recent chemodynamical results of Recchi et al. (2001), suggesting that the wind is differential, are adopted. This implies that the ejecta of type Ia SNe and intermediate-mass stars are lost from the parent galaxy more easily than type II SN ejecta, so that the $\mathrm{Fe}$ and $\mathrm{N}$ ejection efficiencies are larger than the ejection efficiencies of $\alpha$-elements (e.g. $\mathrm{O}, \mathrm{Mg}$ ). This is due to the fact that the type Ia $\mathrm{SNe}$ can transfer into the ISM more energy than the type II SNe, since 


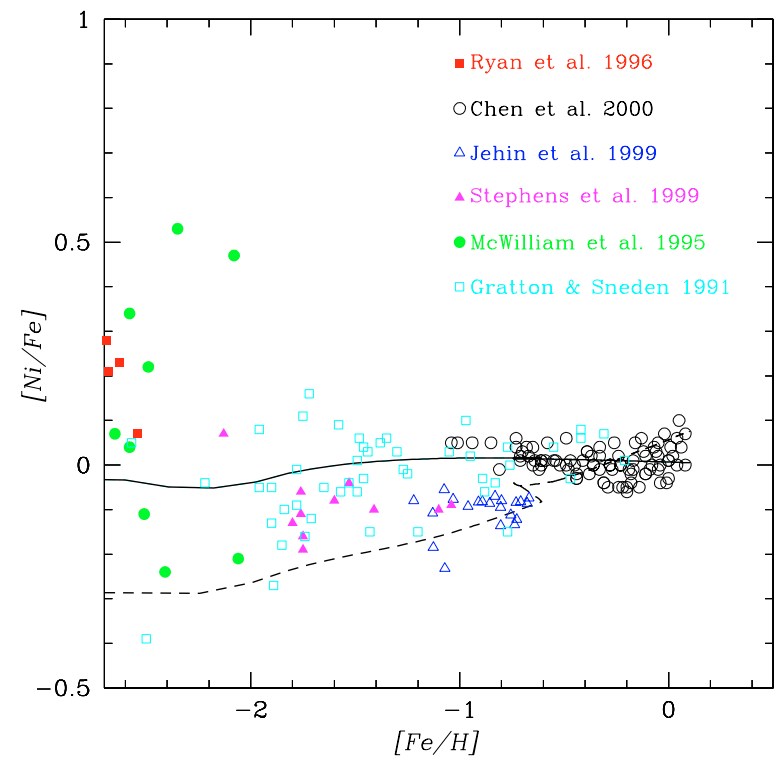

Fig. 12. Observed and predicted $[\mathrm{Ni} / \mathrm{Fe}]$ versus $[\mathrm{Fe} / \mathrm{H}]$ distributions in the solar neighborhood for two different nucleosynthetic prescriptions for the production of $\mathrm{Ni}$. The dashed line corresponds to the model with $\beta=0.0065$ and the solid line to the model with $\beta=0.0049$, where $\beta$ is a multiplicative factor between the Fe and Ni yields (see text). The solid squares are the data from Ryan et al. (1996), the open circles the ones from Chen et al. (2000), the open triangles the ones from Jehin et al. (1999), the filled triangles the ones from Stephens (1999), the filled circles the ones from McWillian et al. (1995), and the open squares the ones from Gratton \& Sneden (1991).

they explode in an already rarefied and hot medium. The IMF is taken from Salpeter (1955).

For the comparison of the "dwarf irregular" model with our observations of DLA systems, we assume that these high redshift galaxies have undergone a single burst of SF. This can be justified by the fact that the DLA galaxies are probably young galaxies. We thus need to essentially constrain three free parameters, the burst star formation efficiency, $v$, the burst duration, $\Delta t$, and the time of occurrence of the burst, $t_{\mathrm{b}}$.

\subsubsection{Stellar yields}

We describe here the nucleosynthesis prescriptions adopted in both the "spiral" and "dwarf irregular" models. They include the yields of Nomoto et al. (1997a) for massive stars $(M>$ $10 M_{\odot}$ ), the yields of van den Hoeck \& Groenwegen (1997) for low- and intermediate-mass stars $\left(0.8 \leq M / M_{\odot} \leq 8\right)$ and the yields of Nomoto et al. (1997b) for type Ia SNe (model W7). For $\mathrm{Zn}$ and Ni we consider the following specific prescriptions.

The nucleosynthesis of $\mathrm{Zn}$ is a debated issue, since what process is making $\mathrm{Zn}$ is rather uncertain. According to stellar models, the production of $\mathrm{Zn}$ can ensue via s-processes in lowand high-mass stars during He-core burning as well as during explosive nucleosynthesis events occurring in type Ia and II $\mathrm{SNe}$ (Matteucci et al. 1993, and references therein). This uncertainty in the $\mathrm{Zn}$ production has repercussions on the reliability of $\mathrm{Zn}$ yields. In this paper we use the $\mathrm{Zn}$ yields adopted by Calura et al. (2003), which were estimated by extrapolating the
Galactic star abundance observations in the solar neighborhood to the high redshift Universe, assuming Fe and $\mathrm{Zn}$ track each other as observed in the metallicity range where the DLAs lie (Prochaska et al. 2000; Mishenina et al. 2002).

The Ni yields have also some difficulties to reproduce the Galactic star abundance observations. Therefore, similarly to $\mathrm{Zn}$, we assumed for the Ni production the results of Matteucci et al. (1993), in which a good fit to the solar abundance of $\mathrm{Ni}$ is found if the bulk of its production is ascribed to type Ia SNe. However, since a non-negligible fraction of $\mathrm{Fe}$ also comes from type II SNe (Nomoto et al. 1997a) and since the abundance of Fe seems to vary in lockstep with that of $\mathrm{Ni}$ in the solar vicinity, the type II SNe should also produce some Ni. In our models the amount of Ni produced through explosive nucleosynthesis in massive stars scales with the Fe yields, according to $Y_{\mathrm{Ni}}=\beta \times Y_{\mathrm{Fe}}$, where $\beta$ represents a multiplicative factor. We have run a chemical evolution model for the solar vicinity varying $\beta$ in order to reproduce the $[\mathrm{Ni} / \mathrm{Fe}]$ versus $[\mathrm{Fe} / \mathrm{H}]$ distribution observed in Galactic stars of different metallicities. Figure 12 shows this $[\mathrm{Ni} / \mathrm{Fe}]$ versus $[\mathrm{Fe} / \mathrm{H}]$ distribution compared with the predictions for the solar neighborhood model, when two values of $\beta$ are adopted (dashed line: $\beta=0.0065$; solid line: $\beta=0.0049$ ). The choice of $\beta=0.0049$ gives a satisfactory agreement between the predictions and the observations, in particular in the metallicity range occupied by the DLAs $([\mathrm{Fe} / \mathrm{H}] \leq-2)$. For $\mathrm{Ni}$ produced in type Ia SNe, we assume a constant value $M_{\mathrm{Ni}} \sim 2.3 \times 10^{-2} M_{\odot}$.

\subsection{Comparing the DLA abundances with the models}

To be able to constrain the star formation history of DLAs from the comparison of their abundance patterns with chemical evolution models, we first need to understand how the abundance patterns can provide information on the star formation history of galaxies. In few words, the absolute abundances depend on the model assumptions (i.e. the SFH), whereas the relative abundances depend only on the nucleosynthesis, the stellar lifetimes and the IMF. Relative abundances can therefore be used as cosmic clocks if they involve two elements formed on different timescales, like it is the case of the $\alpha$-element over Fe-peak element ratios and the $\mathrm{N}$ over $\alpha$-element ratios. As a consequence, these abundance ratios when examined together with the absolute abundances $[\mathrm{Fe} / \mathrm{H}]$, or any other metallicity tracer such as $[\mathrm{Zn} / \mathrm{H}]$, and $[\alpha / \mathrm{H}]$, respectively, completely determine the star formation history of a galaxy. On the other hand, when these abundance ratios are examined as a function of the redshift, they provide constraints on the age of a galaxy, defined as the epoch at which the galaxy has started to form stars (Matteucci 2001).

The relative abundances that we have at disposal in the DLA systems studied here are [Si/Fe], [S/Zn], [S/Fe], [Mg/Fe], $[\mathrm{Ni} / \mathrm{Fe}],[\mathrm{N} / \mathrm{Si}]$ and $[\mathrm{N} / \mathrm{S}]$. For each DLA individually, we consider all of them at once to first constrain the SFH and then the age of the associated galaxy by means of a detailed comparison with a grid of chemical evolution models. This grid includes the "spiral" model computed at different galactocentric radii from $R=2$ to $18 \mathrm{kpc}$ and the "dwarf irregular" model computed 
for a single burst of SF with different characteristics defined by varying the three free parameters $v, \Delta t$ and $t_{\mathrm{b}}$. The adopted cosmology is $H_{0}=65 \mathrm{~km} \mathrm{~s}^{-1} \mathrm{Mpc}^{-1}$ (and hence $h=0.65$ ), $\Omega_{\mathrm{M}}=0.3$ and $\Omega_{\Lambda}=0.7$.

To determine the best chemical evolution model reproducing the abundance patterns observed in each DLA system by taking into account all the different informations available on the system, we use a statistical test when the best solution cannot be clearly identified by eye. This test consists first in determining the minimal distance between the data point and the curve of a given chemical model in each abundance diagram at disposal. For this purpose, we consider the $1 \sigma$ error on the data point (or more precisely the covariance matrix of the $1 \sigma$ measurement error), and we derive this minimal distance by computing the distances $d_{i}$ from the data point to the points defining the considered model curve and by looking for the $d_{i}$ for which the $d_{i} / \sigma_{i}$ ratio is minimal. Second, once the minimal distances for all the abundance diagrams considered in each system and for a given model are derived, we compute their weighted mean. Finally, the comparison of the weighted means obtained for different chemical models determines the best chemical evolution model which represents the data points and thus the DLA galaxy. The upper and lower limits are not taken into account in this test.

\subsection{1. $\mathrm{Q} 0100+13, z_{\mathrm{abs}}=2.309$}

To determine the nature of this DLA galaxy, we have five different abundance ratios at disposal. We can thus try to constrain the best chemical evolution model which reproduces this DLA galaxy by taking into account the information provided by the following five diagrams: $[\mathrm{S} / \mathrm{Zn}]$ versus $[\mathrm{Zn} / \mathrm{H}],[\mathrm{S} / \mathrm{Fe}]$ versus $[\mathrm{Fe} / \mathrm{H}],[\mathrm{Mg} / \mathrm{Fe}]$ versus $[\mathrm{Fe} / \mathrm{H}],[\mathrm{Ni} / \mathrm{Fe}]$ versus $[\mathrm{Fe} / \mathrm{H}]$ and $[\mathrm{N} / \mathrm{S}]$ versus $[\mathrm{S} / \mathrm{H}]$. For the absolute and relative abundances of refractory elements we consider the dust-corrected values as derived with the dust correction method of Vladilo (2002a,b, and see Tables 8 and 9). In this DLA system, however, the dust corrections are small, since the $[\mathrm{Zn} / \mathrm{Fe}]$ ratio is close to solar $(=+0.25 \pm 0.04)$.

We considered the "spiral" model at the galactocentric radii $R=6,8,12$, and $18 \mathrm{kpc}$. The "spiral" model at $R=8 \mathrm{kpc}$ best reproduces the five abundance ratios measured in this high redshift galaxy (see the thick dashed-dotted curves in Fig. 13). We might be satisfied with this solution, since the model curves are in agreement with the data points within less than $1 \sigma$ in four out of the five diagrams. In the case of the $\mathrm{Ni} / \mathrm{Fe}$ ratio, the model is in agreement only within $2-3 \sigma$. But, to have a complete picture and to check the uniqueness of the derived solution, we investigated whether a "dwarf irregular" model can also correctly reproduce the data points. We considered a "dwarf irregular" model with a single burst of SF and explored the following values for the three parameters characterizing the burst: $v=0.5,0.7,0.9,1 \mathrm{Gyr}^{-1}, \Delta t=0.05,0.07,0.1,0.2 \mathrm{Gyr}$ and $t_{\mathrm{b}}=1,1.1,1.5,2$ Gyr. The "dwarf irregular" model which best reproduces the data points has one burst with a star formation efficiency $v=0.9 \mathrm{Gyr}^{-1}$ and a short duration $\Delta t=0.07$ Gyr (see the thin solid curves in Fig. 13).
The burst occurs after $t_{\mathrm{b}}=2 \mathrm{Gyr}$ of continuous infall of pristine gas. These "dwarf irregular" model characteristics very well match the starburst parameters determined for the BCGs (e.g. Lanfranchi \& Matteucci 2003). In summary, two chemical evolution models yet tracing different star formation histories correctly reproduce the abundance ratio versus metallicity distributions observed in the DLA toward Q0100+13. The "spiral" model at $R=8 \mathrm{kpc}$ has a continuous SFH, whereas the "dwarf irregular" model constrained by the observations has a bursting SFH (see Sect. 6.1). The identification of the best model among these two is very difficult, because at this intermediate metallicity both models yield very similar chemical abundance patterns. The distinction is more pronounced at $[\mathrm{Fe} / \mathrm{H}]<-2$ or at $[\mathrm{Fe} / \mathrm{H}]>-1$.

We carried out the investigation of the age of the system, namely the most likely redshift of formation, $z_{\mathrm{f}}$, of the system, for the two models. For the "spiral" model at $R=8 \mathrm{kpc}$, we analyzed the $[\mathrm{Zn} / \mathrm{H}],[\mathrm{Ni} / \mathrm{Fe}],[\mathrm{Mg} / \mathrm{Fe}],[\mathrm{S} / \mathrm{Zn}],[\mathrm{S} / \mathrm{Fe}]$ and $[\mathrm{N} / \mathrm{S}]$ versus redshift diagrams. The estimated redshift of formation is between $z_{\mathrm{f}}=2.4-3$ with the best solution being at $z_{\mathrm{f}} \sim 2.5$ which is in excellent agreement with all the data points (see the thick dashed-dotted curves in Fig. 14). The DLA system is observed at $z_{\mathrm{abs}}=2.309$, hence $z_{\mathrm{f}} \sim 2.5$ corresponds to an age of the DLA galaxy of $0.25 \pm 0.20 \mathrm{Gyr}$. In the case of the "dwarf irregular" model, the determination of the age is more complex, because this model has several free parameters. The parameter having the lowest impact is the time of occurrence of the burst, $t_{\mathrm{b}}$, according to the adopted definition of the age (see Sect. 6.2). In addition, $t_{\mathrm{b}}$ is difficult to determine, whereas the star formation efficiency, $v$, and the burst duration, $\Delta t$, can be robustly constrained by the observations. It is the burst duration which has the largest weight in the determination of the age of the system. We satisfactorily reproduce the data points (within $1 \sigma$ ) for $\Delta t$ values between 0.05 and $0.20 \mathrm{Gyr}$. Hence, we feel confident in concluding that the possible age of the system is between $0.05-0.20$ Gyr. Given the assumption that the galaxy has undergone a single burst of SF, the corresponding redshift of formation is between 2.35-2.47.

Both models point to a young age for the DLA galaxy, lower than $250 \mathrm{Myr}$. This age is consistent with the $\alpha$-element over Fe-peak element enhancement observed in this DLA system (see Sect. 5.3), which indicates that the enrichment of this system is dominated by massive star products and requires a minimal contribution from type Ia SNe. In addition, we saw in Sect. 5.3 that the N/S ratio in this DLA system is relatively high, very close to the primary $\mathrm{N}$ "plateau", $[\mathrm{N} / \mathrm{S}]=$ $-0.83 \pm 0.14$. This $\mathrm{N} / \mathrm{S}$ ratio and the estimated age for the DLA galaxy provide important constraints on the $\mathrm{N}$ stellar progenitors. Indeed, such a high N/S value can be reached within less than $250 \mathrm{Myr}$, only if $\mathrm{N}$ is produced by intermediate-mass stars with masses between 5-8 $M_{\odot}$, which have lifetimes between 30 and $70 \mathrm{Myr}$, and by massive stars. Chiappini et al. (2003) recently showed that the same chemical evolution models as those used in this paper, but computed with the recent published stellar yields of Meynet \& Maeder (2002) which take into account the effects of rotation in the stellar evolution, still reproduce such high $\mathrm{N} / \mathrm{S}$ values within a timescale lower than 250 Myr. 

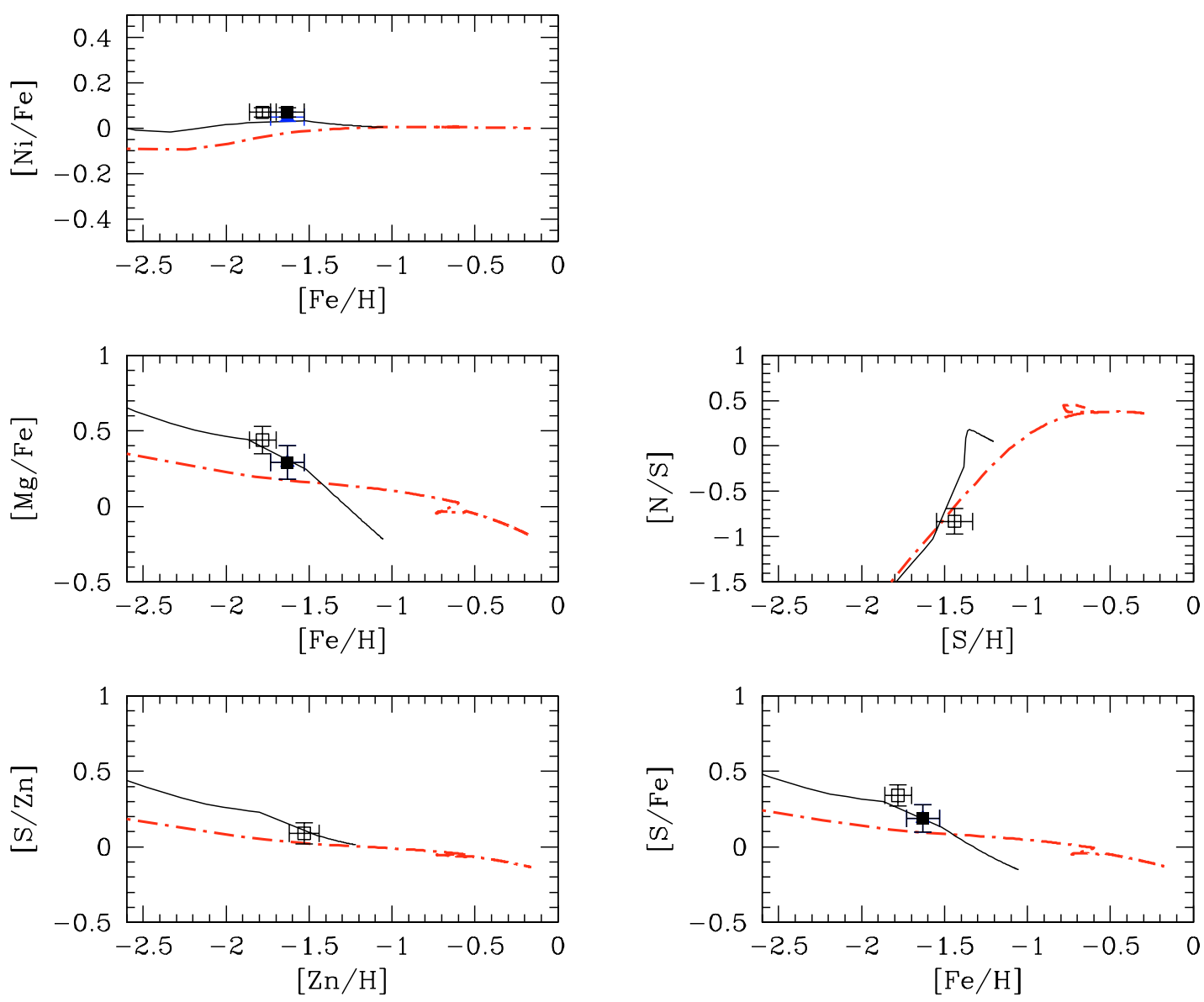

Fig. 13. Observed and predicted abundance ratios versus metallicity for the DLA at $z_{\text {abs }}=2.309$ toward Q0100+13. The thick dashed-dotted curves correspond to the "spiral" model at $R=8 \mathrm{kpc}$. The thin solid curves correspond to the "dwarf irregular" model with a single burst of SF occurring at $t_{\mathrm{b}}=2 \mathrm{Gyr}$ and with a star formation efficiency $v=0.9 \mathrm{Gyr}^{-1}$ and a duration $\Delta t=0.07$ Gyr. In this and all the following figures, the open squares represent the measured abundance ratio measurements, and the filled triangles and the filled squares represent the abundance ratios corrected for dust depletion according to the Vladilo (2002a) method using the models E00 and E11, respectively.

\subsection{2. $\mathrm{Q} 1331+17, \mathrm{zabs}_{\mathrm{a}}=1.776$}

We have collected a lot of information on this DLA system. The observed abundance ratios at our disposal are $[\mathrm{S} / \mathrm{Zn}],[\mathrm{S} / \mathrm{Fe}]$, $[\mathrm{Si} / \mathrm{Fe}],[\mathrm{Mg} / \mathrm{Fe}],[\mathrm{Ni} / \mathrm{Fe}]$ and the upper limits on $[\mathrm{N} / \mathrm{S}]$ and $[\mathrm{N} / \mathrm{Si}]$. This DLA system has a very high $[\mathrm{Zn} / \mathrm{Fe}]=0.75 \pm$ 0.05 ratio and exhibits one of the largest dust depletion level of any DLA. In Fig. 15 we can see the high differences between the observed and dust-corrected values.

First, we examined the "spiral" model at the galactocentric radii $R=8,12,16$ and $18 \mathrm{kpc}$. The best model reproducing the data points in the $[\mathrm{S} / \mathrm{Zn}]$ versus $[\mathrm{Zn} / \mathrm{H}],[\mathrm{S} / \mathrm{Fe}]$ versus $[\mathrm{Fe} / \mathrm{H}],[\mathrm{Si} / \mathrm{Fe}]$ versus $[\mathrm{Fe} / \mathrm{H}],[\mathrm{Mg} / \mathrm{Fe}]$ versus $[\mathrm{Fe} / \mathrm{H}]$ and $[\mathrm{Ni} / \mathrm{Fe}]$ versus $[\mathrm{Fe} / \mathrm{H}]$ diagrams is the "spiral" model of outer regions of the disk, at $R=16 \mathrm{kpc}$ (see the thick dashed-dotted curves in Fig. 15). The model curves are in excellent agreement with the data points within less than $1 \sigma$ in the five diagrams. Secondly, we investigated the "dwarf irregular" model to check the uniqueness of the solution. We considered the "dwarf irregular" model with a single burst of SF and explored a large range of values for the three parameters characterizing the burst. We found out that the "dwarf irregular" model with a bursting SFH cannot reproduce the observations, which show solar $\alpha$-element over Fe-peak element ratios, because the bursting SFH predicts $\alpha$-enhanced patterns. On the other hand, the "dwarf irregular" model with a continuous SFH characterized by a single burst of SF with a star formation efficiency in the range $v=0.02-0.09 \mathrm{Gyr}^{-1}$, with the best value being $v=0.03 \mathrm{Gyr}^{-1}$, and a duration over the whole Hubble time satisfactorily reproduces the observations (see the thin solid curves in Fig. 15).

Again none of these two models having a different star formation history - the "spiral" model of outer regions of the disk has a bursting SFH (see Sect. 6.1.1), while the "dwarf irregular" model constrained by the observations has an inefficient continuous SFH - can be easily discarded, because they yield similar chemical abundance patterns due to the fact that they both are characterized by a weak SF. The $\mathrm{Mg} / \mathrm{Fe}$ ratio could be used as a discriminant between the two models (see Fig. 15), but there are substantial uncertainties in the $\mathrm{Mg}$ yields (e.g. Chiappini et al. 1999).

We investigated the age of the DLA galaxy for the two models. For this purpose, we considered the $[\mathrm{Zn} / \mathrm{H}],[\mathrm{S} / \mathrm{Zn}]$, $[\mathrm{S} / \mathrm{Fe}],[\mathrm{Si} / \mathrm{Fe}],[\mathrm{Mg} / \mathrm{Fe}]$ and $[\mathrm{Ni} / \mathrm{Fe}]$ versus redshift diagrams. 

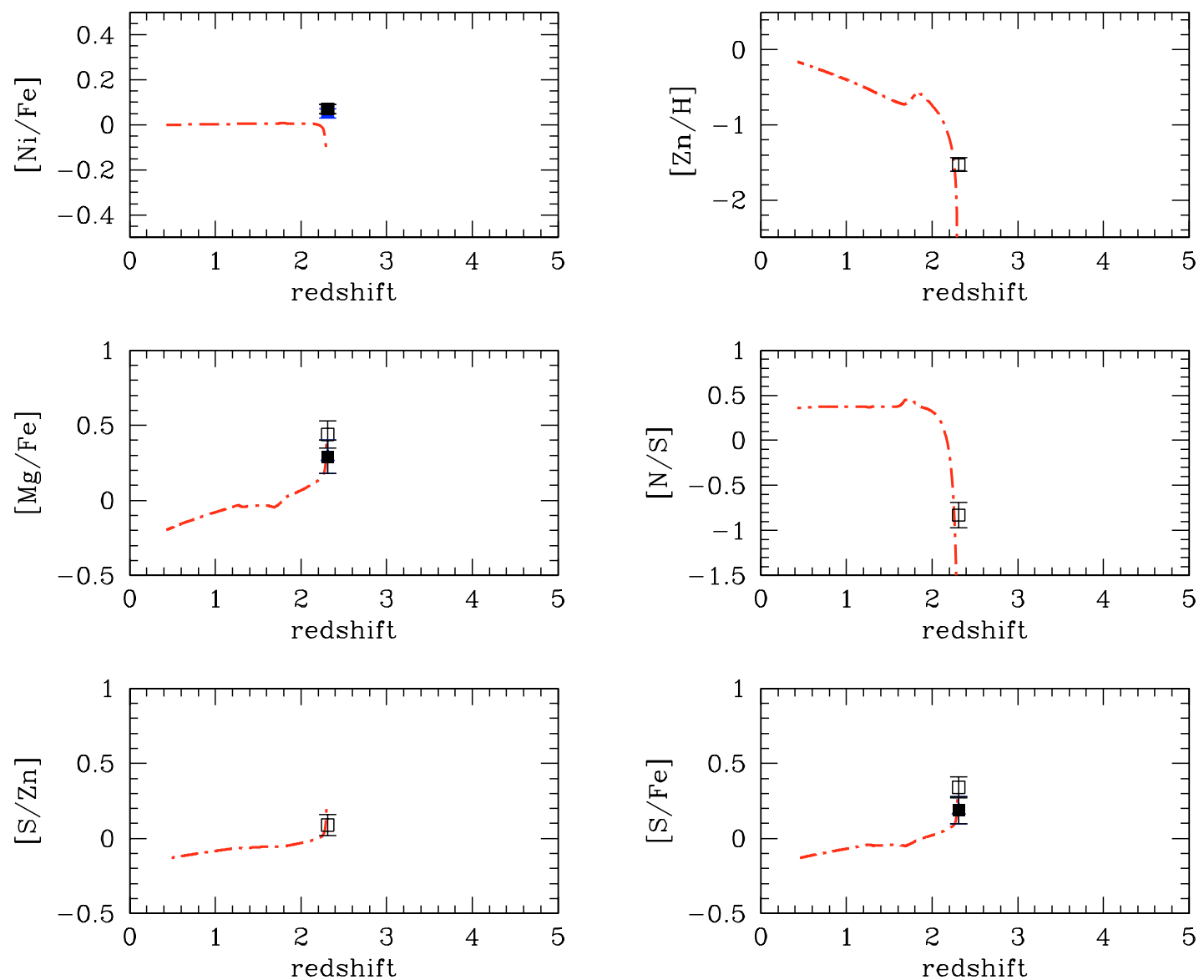

Fig. 14. Observed and predicted abundance ratios versus redshift for the DLA at $z_{\mathrm{abs}}=2.309$ toward Q0100+13. The thick dashed-dotted curves correspond to the "spiral" model at $R=8 \mathrm{kpc}$ and a redshift of formation $z_{\mathrm{f}}=2.5$. For the definition of symbols, see Fig. 13 .

The range of redshifts of formation determined for the "spiral" model at $R=16 \mathrm{kpc}$ is $z_{\mathrm{f}}=2.3-3$, with the best solution being at $z_{\mathrm{f}} \sim 2.8$ (see the thick dashed-dotted curves in Fig. 16). For the "dwarf irregular" model with an inefficient continuous SF rate, the estimated redshift of formation is large, roughly around $z_{\mathrm{f}} \sim 10$ (see the thin solid curves in Fig. 16). The DLA system is observed at $z_{\mathrm{abs}}=1.776$, hence $z_{\mathrm{f}} \sim 2.8$ and $z_{\mathrm{f}} \sim 10$ correspond to an age of the DLA galaxy of $1.5 \pm 0.4 \mathrm{Gyr}$ and $\gtrsim 3.5 \mathrm{Gyr}$, respectively.

According to the recent WMAP results, the reionization seems to have occurred at a redshift considerably higher than what was thought previously, namely at $z_{\text {reion }}=20_{-9}^{+10}$ (Bennett et al. 2003). Therefore, if the intergalactic medium has been reionized by the first stars formed in very young galaxies, a redshift of the order of 10 could be reasonable for the appearance of the first galactic structures in the Universe. The derived solution that the associated galaxy with the DLA system at $z_{\mathrm{abs}}=1.776$ toward Q1331+17 may be a dwarf irregular galaxy with a continuous SFH formed at $z_{\mathrm{f}} \sim 10$ thus is possible. The solution that the associated galaxy is a spiral galaxy with the QSO line of sight crossing the outer regions of its disk at $R=16 \mathrm{kpc}$ and an age of $1.5 \mathrm{Gyr}$ is also plausible. Both models point to a large age for this DLA galaxy. This reflects the long timescale necessary to accrete the gas as well as to reach the observed solar $\alpha$-element over Fe-peak element ratios (see Sect. 5.3) which require a substantial contribution from type Ia SNe, releasing the Fe-peak elements only after $10^{8}-10^{9} \mathrm{yrs}$. But, both models fail in reproducing the measured $[\mathrm{N} / \mathrm{S}]$ and $[\mathrm{N} / \mathrm{Si}]$ upper limits, since they predict almost solar $[\mathrm{N} / \mathrm{S}, \mathrm{Si}]$ ratios at $[\mathrm{S}, \mathrm{Si} / \mathrm{H}] \simeq-1.25$. Such high $\mathrm{N} / \alpha$ values at these low metallicities have never been observed in any DLA and H II region. This suggests that the yields of van den Hoeck \& Groenwegen (1997) may lead to an overestimation of the $\mathrm{N}$ production by intermediate-mass stars at the lower end of masses and by low-mass stars, which had time to release $\mathrm{N}$ in this DLA galaxy, given the inferred long age. In the case of the $\mathrm{N}$ production by massive intermediate-mass stars, the yields of van den Hoeck \& Groenwegen (1997) are more reliable.

\subsection{3. $Q 2231-00, z_{\mathrm{abs}}=2.066$}

In this system, the measured abundance ratios at disposal are $[\mathrm{S} / \mathrm{Zn}],[\mathrm{S} / \mathrm{Fe}],[\mathrm{Si} / \mathrm{Fe}],[\mathrm{Ni} / \mathrm{Fe}]$, and the upper limits on $[\mathrm{N} / \mathrm{Si}]$ and $[\mathrm{N} / \mathrm{S}]$. We consider the dust-corrected values, since nonnegligible dust corrections are required in this system with $[\mathrm{Zn} / \mathrm{Fe}]=+0.45 \pm 0.07$. We first investigated the "spiral" model to reproduce the $[\mathrm{S} / \mathrm{Zn}]$ versus $[\mathrm{Zn} / \mathrm{H}],[\mathrm{S} / \mathrm{Fe}]$ versus $[\mathrm{Fe} / \mathrm{H}],[\mathrm{Si} / \mathrm{Fe}]$ versus $[\mathrm{Fe} / \mathrm{H}]$ and $[\mathrm{Ni} / \mathrm{Fe}]$ versus $[\mathrm{Fe} / \mathrm{H}]$ diagrams. The favored "spiral" model turned out to be the one representing the inner regions of the galactic disk, i.e. at 

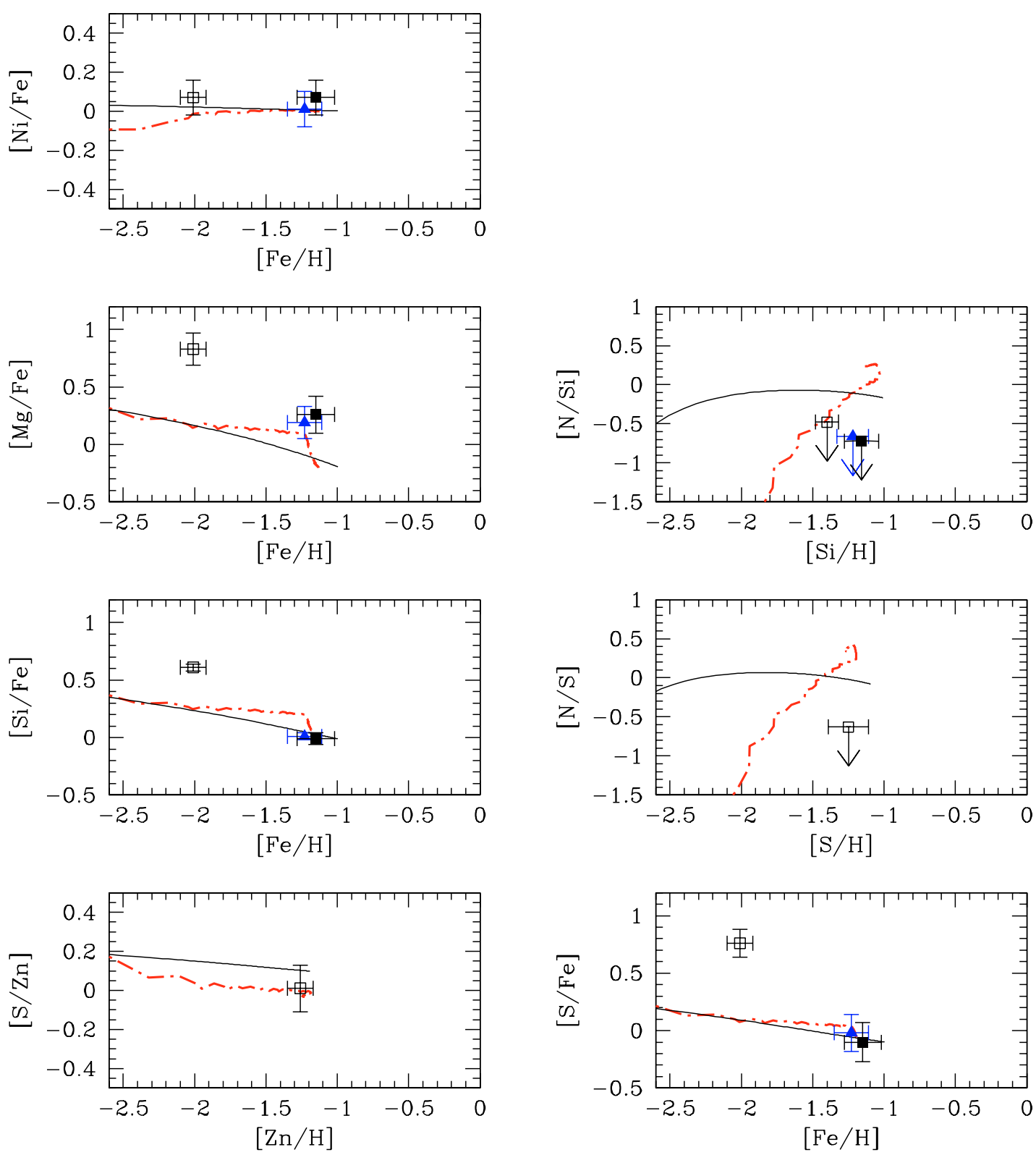

Fig. 15. Observed and predicted abundance ratios versus metallicity for the DLA at $z_{\mathrm{abs}}=1.776$ toward Q1331+17. The thick dashed-dotted curves correspond to the "spiral" model at $R=16 \mathrm{kpc}$ and the thin solid curves correspond to the "dwarf irregular" model with a continuous $\mathrm{SF}$ at an efficiency $v=0.03 \mathrm{Gyr}^{-1}$. For the definition of symbols, see Fig. 13.

galactocentric radii $R<8 \mathrm{kpc}$. In the inner regions of the disk compared to the outer regions, the infall of external gas is faster and leads to higher gas densities, and hence to a more efficient $\mathrm{SF}$ (see Sect. 6.1.1). The $\alpha$-elements thus are more enhanced relative to the Fe-peak elements at metallicities $[\mathrm{Fe} / \mathrm{H}]<-1.5$ dex in the inner regions of the disk than in the outer regions. Despite this, the "spiral" model of inner regions of the disk does not succeed in reproducing all the measured data points (see the thick dashed-dotted curves in Fig. 17). It mainly fails in correctly reproducing the $[\mathrm{S} / \mathrm{Zn}]$ versus $[\mathrm{Zn} / \mathrm{H}]$ and $[\mathrm{S} / \mathrm{Fe}]$ versus $[\mathrm{Fe} / \mathrm{H}]$ data points, and the $[\mathrm{N} / \mathrm{Si}]$ versus $[\mathrm{Si} / \mathrm{H}]$ and $[\mathrm{N} / \mathrm{S}]$ versus $[\mathrm{S} / \mathrm{H}]$ upper limits.

We then considered the "dwarf irregular" model. We assumed a single burst of SF and explored the following values for the three free parameters characterizing the burst: $v=2.8$ $5.0 \mathrm{Gyr}^{-1}, \Delta t=0.05,0.1,0.15 \mathrm{Gyr}$ and $t_{\mathrm{b}}=0.2,0.5,0.8 \mathrm{Gyr}$. The "dwarf irregular" model which best reproduces the data points has one burst with a very high star formation efficiency $v=4.2 \mathrm{Gyr}^{-1}$ and a duration $\Delta t=0.1 \mathrm{Gyr}$ (see the thin solid curves in Fig. 17). The burst occurs after $t_{\mathrm{b}}=0.5 \mathrm{Gyr}$ of continuous infall of pristine gas. This model is the favored one in comparison with the "spiral" model of inner regions of the disk. It reproduces at $1 \sigma$ all the abundance ratios. Only the $[\mathrm{N} / \mathrm{S}]$ upper limit is at odds.

We investigated the age of the DLA galaxy assuming the constrained "dwarf irregular" model. As in the case of the DLA toward Q0100+13, the parameter having the highest impact in the age determination is the duration of the burst. We feel 

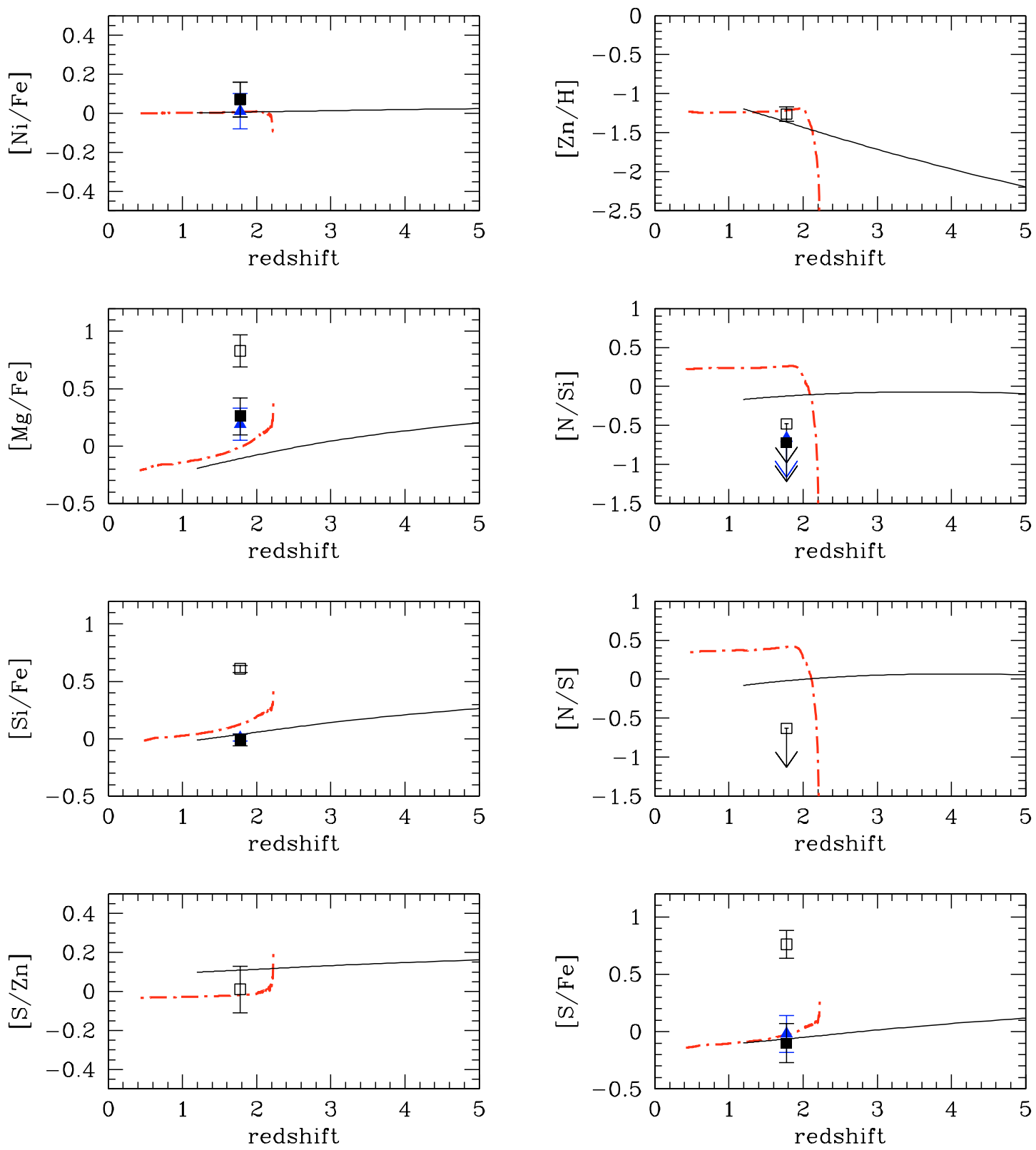

Fig. 16. Observed and predicted abundance ratios versus redshift for the DLA at $z_{\mathrm{abs}}=1.776$ toward Q1331+17. The thick dashed-dotted curves correspond to the "spiral" model at $R=16 \mathrm{kpc}$ and a redshift of formation $z_{\mathrm{f}}=2.8$. The thin solid curves correspond to the "dwarf irregular" model with a continuous SF at an efficiency $v=0.03 \mathrm{Gyr}^{-1}$ and a redshift of formation $z_{\mathrm{f}}=10$. For the definition of symbols, see Fig. 13 .

confident in assuming an age between $0.05-0.15 \mathrm{Gyr}$, corresponding to the range of values explored for $\Delta t$. This yields a redshift of formation between 2.10-2.16 given the assumption that the galaxy has undergone a single burst of SF.

\subsection{Star formation rates}

The star formation is a key parameter in the formation and evolution of galaxies. Therefore, the knowledge of the star formation rate $(S F R)$ of galaxies is very important. Kennicutt (1983) provided the first precise diagnostics for the measure of the $S F R$, such as emission-line fluxes and UV continuum luminosities. Later Madau et al. (1996) reconstructed the cosmic star formation history by measuring the comoving luminosity density of star-forming galaxies as a function of redshift. While the original work of Madau et al. (1996) showed a peak in the cosmic star formation at $z \approx 1-2$, recent results, based on larger samples of galaxies and corrections for dust extinction of the emitted starlight, do not show such a peak. The $S F R$ per unit comoving volume increases by a factor of $\sim 10$ in the redshift interval $z=[0,1]$, and then either remains constant at $z>1$ up to $z=6$ (Steidel et al. 1999) or keeps increasing to even higher redshifts (Lanzetta et al. 2002; Calura \& Matteucci 2003). However, the galaxies from which these results are derived are unlikely to be representative of the bulk of the galaxy population in the Universe. Indeed, whereas the $S F R$ per unit area for the Milky Way is $\psi \sim 4 \times 10^{-3} M_{\odot} \mathrm{yr}^{-1} \mathrm{kpc}^{-2}$ (Kennicutt 1998), the comoving $S F R$ at $\sim 3$ is inferred from 

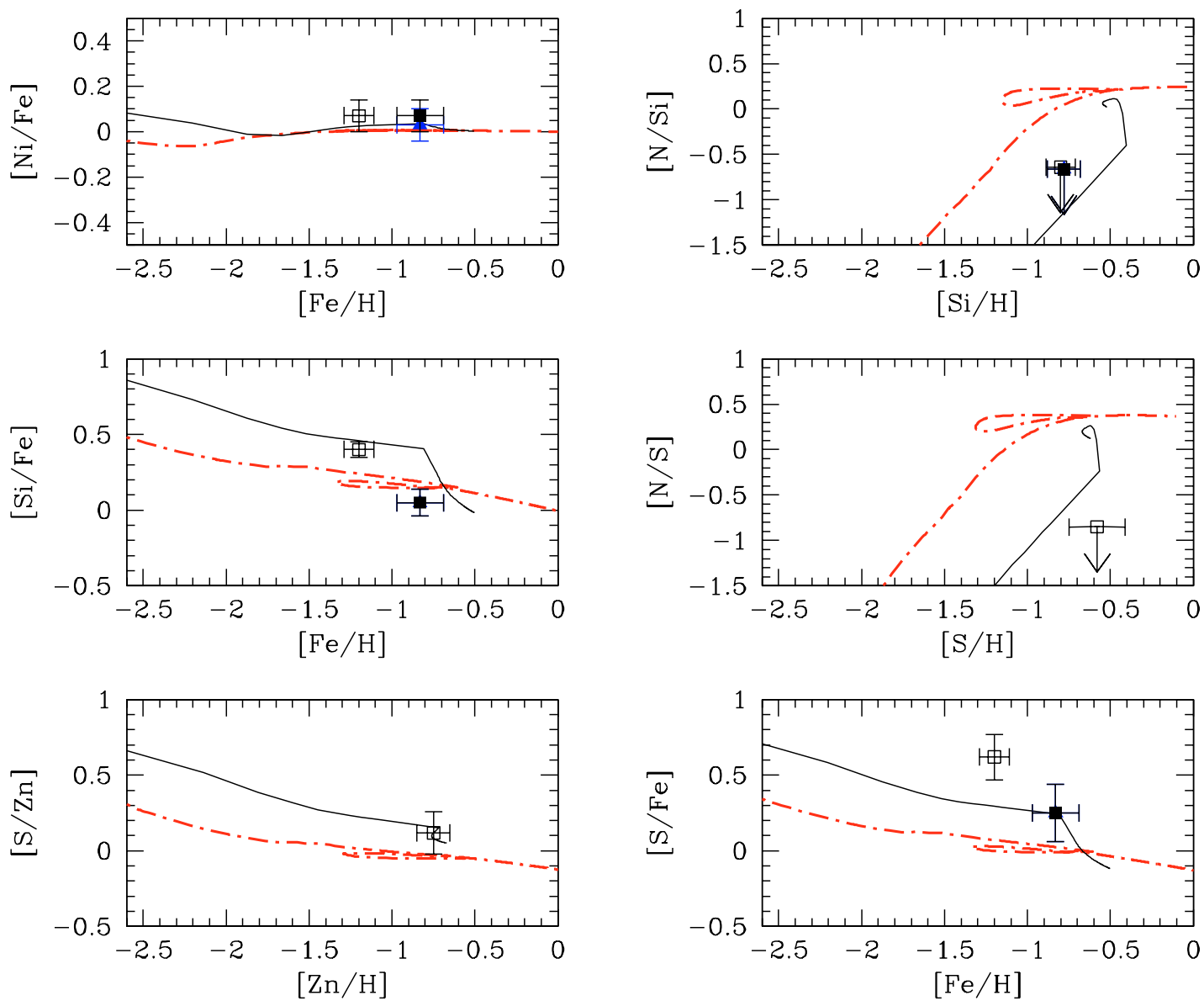

Fig. 17. Observed and predicted abundance ratios versus metallicity for the DLA at $z_{\mathrm{abs}}=2.066$ toward Q2231-00. The thick dashed-dotted curves correspond to the "spiral" model at $R=2 \mathrm{kpc}$. The thin solid curves correspond to the "dwarf irregular" model with a single burst of SF occurring at $t_{\mathrm{b}}=0.5 \mathrm{Gyr}$ and with a star formation efficiency $v=4.2 \mathrm{Gyr}^{-1}$ and a duration $\Delta t=0.1$ Gyr. For the definition of symbols, see Fig. 13.

Lyman-break galaxies, a highly luminous population of starforming objects in which $\psi \geq 1 M_{\odot} \mathrm{yr}^{-1} \mathrm{kpc}^{-2}$ (Pettini et al. 2001). As a result, the existing measurements of the cosmic star formation history take into account the contribution of only highly luminous and star-forming galaxies. The access to the star formation rates in other types of galaxies thus is crucial.

The DLA galaxy population is ideal for this purpose. Indeed, these objects sample various types of galaxies over a large range of lookback times (see Sects. 6.2.1-6.2.3), since they are detected independently of their distance, luminosity and SFH. In addition, because the DLAs are not drawn from a flux limited sample of galaxies, we are able to derive the $S F R$ values below those determined from radiation emitted by starforming galaxies. Recently, Wolfe et al. (2003a,b) provided the first estimations of SFRs per unit area in DLAs. Their technique to infer the SFRs consists in determining the rate at which the neutral gas in DLAs is heated.

In this work, we also have access to the SFRs of DLAs. Indeed, in the case of the "spiral" model the SFR per unit area is a direct output of the model, and in the case of the "dwarf irregular" model the different physical quantities correspond to the absolute values, thus to derive the $S F R$ per unit area we assume a spherical symmetry and a galactic radius of $5 \mathrm{kpc}$.
The derived star formation rates per unit area for the DLAs studied are given in Table 11, and are between $-2.1<\log \psi<$ $-1.5 M_{\odot} \mathrm{yr}^{-1} \mathrm{kpc}^{-2}$. They were obtained for the models constrained in the previous sections (Sects. 6.2.1-6.2.3). They correspond in the case of the "spiral" model to the SFR that the DLA galaxy has at the time of its observations and in the case of the "dwarf irregular" model to the average $S F R$ integrated over the time of the burst of SF, i.e. over the period when the $\mathrm{SF}$ is active. The errors on the derived $S F R$ s are very difficult to estimate. Our SFR measurements are in agreement with the interval of $S F R$ values obtained by Wolfe et al. $(2003 \mathrm{a}, \mathrm{b})^{2}$ in DLAs using a completely different technique. We thus confirm that the SFRs per unit area in DLAs are moderate and similar to that measured in the Milky Way disk.

\section{Conclusions}

The damped $\operatorname{Ly} \alpha$ systems are our best laboratory to study the high redshift galaxies. Indeed, accurate chemical abundances of these systems can be obtained over a large interval of cosmic

2 The SFRs obtained by Wolfe et al. (2003a,b) correspond to the $S F R$ values that the DLAs have at the time of their observations. 
Table 11. Determination of the star formation rates.

\begin{tabular}{lcc}
\hline \hline DLA system & $\begin{array}{c}S F R \text { for the best "spiral" model } \\
{\left[M_{\odot} \mathrm{yr}^{-1} \mathrm{kpc}^{-2}\right]}\end{array}$ & $\begin{array}{c}S F R \text { for the best "dwarf irregular" model } \\
{\left[M_{\odot} \mathrm{yr}^{-1} \mathrm{kpc}^{-2}\right]}\end{array}$ \\
\hline $\mathrm{Q} 0100+13, z_{\mathrm{abs}}=2.309$ & $8.5 \times 10^{-3}$ & $1.0 \times 10^{-2}$ \\
$\mathrm{Q} 1331+17, z_{\mathrm{abs}}=1.776$ & $7.5 \times 10^{-3}$ & $9.2 \times 10^{-5}$ \\
$\mathrm{Q} 2231-00, z_{\mathrm{abs}}=2.066$ & - & $4.0 \times 10^{-2}$ \\
\hline
\end{tabular}

time, and they thus offer the best opportunity to track the chemical evolution of galaxies in the Universe. The DLA galaxy population has until now been analyzed as a whole and chemical evolution models were constructed in order to interpret the abundance patterns of these objects as an ensemble, considering them as an evolutionary sequence. However, several pieces of evidence show that the DLAs likely trace galaxies of different types and with different evolutionary histories. At this stage of knowledge, one would like to define more precisely the star formation histories and the chemical evolution stages sampled by these objects. Therefore, we aimed at constructing a sample of DLAs for which one would be able to constrain the star formation history, the age and the star formation rate of each system individually. For this purpose, it is first imperative to obtain comprehensive sets of elemental abundances.

By combining our UVES-VLT spectra of a sample of four DLAs in the redshift interval $z_{\text {abs }}=1.7-2.5$ toward the quasars Q0100+13, Q1331+17, Q2231-00 and Q2343+12 with the existing HIRES-Keck spectra, we covered the total optical spectral range from 3150 to $10000 \AA$ for the four quasars. Thanks to this large wavelength coverage and the high quality of the spectra, we succeeded in measuring the column densities of up to 21 ions, namely of 15 elements $-\mathrm{N}, \mathrm{O}, \mathrm{Mg}, \mathrm{Al}, \mathrm{Si}, \mathrm{P}$, $\mathrm{S}, \mathrm{Cl}, \mathrm{Ar}, \mathrm{Ti}, \mathrm{Cr}, \mathrm{Mn}, \mathrm{Fe}, \mathrm{Ni}, \mathrm{Zn}$. With the detections of adjacent ions of the same element, such as $\mathrm{Al}^{+} / \mathrm{Al}^{++}, \mathrm{Fe}^{+} / \mathrm{Fe}^{++}$ and $\mathrm{N}^{0} / \mathrm{N}^{+}$, and of the $\mathrm{Ar} / \mathrm{Si}, \mathrm{S}$ ratios, we constrained the photoionization effects, which may affect the DLA gas-phase abundances. Our analysis revealed that the DLA toward Q2343+12 requires important ionization corrections, while in the three other DLAs the ionization corrections are negligible. With the detection of both refractory and mildly/non-refractory elements of the same nucleosynthetic origin, we evaluated the dust depletion effects and computed the dust corrections using the method of Vladilo (2002a,b). The dust corrections are particularly important in the DLA toward Q1331+17. This system with $[\mathrm{Zn} / \mathrm{Fe}]=+0.75 \pm 0.05$ exhibits one of the largest dust depletion level of any DLA. The constraint of both the photoionization and dust depletion effects allowed us to determine relatively robust intrinsic chemical abundance patterns of three out of the four DLAs studied.

The intrinsic chemical abundance patterns are the signature of the star formation history of galaxies, which one needs to correctly interpret. For this purpose, we called on the chemical evolution models for spiral and dwarf irregular galaxies (see Calura et al. 2003). By comparing these models with the distributions of the abundance ratios of two elements produced on different timescales as a function of the metallicity we can constrain the star formation history of a DLA galaxy, and with the distributions of the same abundance ratios considered as a function of the redshift we can constrain the age of a galaxy. The access to several abundance ratios, $[\mathrm{S} / \mathrm{Zn}],[\mathrm{S} / \mathrm{Fe}],[\mathrm{Si} / \mathrm{Fe}]$, $[\mathrm{O} / \mathrm{Zn}],[\mathrm{Mg} / \mathrm{Fe}],[\mathrm{Ni} / \mathrm{Fe}],[\mathrm{N} / \mathrm{S}],[\mathrm{N} / \mathrm{Si}]$ and $[\mathrm{N} / \mathrm{O}]$, in the same DLA allowed us for the first time to determine the star formation history and the age of three DLA systems. We were also able to estimate the star formation rates per unit area of the DLAs, since the SFR is a direct output of the chemical evolution models, once they are constrained. Our results show that the galaxy associated with the DLA toward Q0100+13 is either a spiral galaxy showing similar characteristics as those observed in the solar neighborhood $(R \sim 8 \mathrm{kpc})$ or a dwarf irregular galaxy with a single burst of SF with properties similar to the ones observed in the BCGs. It is a young galaxy with an age of $250 \pm 200 \mathrm{Myr}$ or between 50-200 Myr, respectively. The galaxy associated with the DLA toward Q1331+17 is very likely a spiral galaxy observed in the outer regions of its disk $(R \sim 16 \mathrm{kpc})$ and $1.5 \pm 0.4$ Gyr old. But, it can also well be a dwarf irregular galaxy with an inefficient continuous SFH and an age $\gtrsim 3.5$ Gyr. Finally, the galaxy associated with the DLA toward Q2231-00 is a dwarf irregular galaxy with a single intense burst of SF and an age between 50-150 Myr. The estimated star formation rates per unit area of these three objects are between $-2.1<\log \psi<-1.5 M_{\odot} \mathrm{yr}^{-1} \mathrm{kpc}^{-2}$. They are in agreement with the values obtained by Wolfe et al. (2003a,b) in DLAs using a different technique, and similar to the SFR per unit area measured in the Milky Way disk.

This work shows that the DLAs studied may either be associated with the outer regions of disks of spiral galaxies $(R \geq 8 \mathrm{kpc})$ or with dwarf irregular galaxies with bursting or continuous star formation histories. Observed at redshifts $z_{\mathrm{abs}}=1.7-2.5$, they may be very young galaxies with ages between 50-250 Myr, but also galaxies with ages larger than $>1.5$ Gyr. Although this work needs to be extended to a larger sample of DLA systems to be representative of the overall population of DLAs at high redshift, we can already derive a number of conclusions. Our limited sample is centered at $z \sim 2$, a redshift region where spectroscopic data on light-emitting galaxies just start to become available. We do not find any association of DLAs with episodes of massive star formation, the derived star formation rates per unit area are moderate. Clearly this is not the standard scenario, and we cannot exclude this possibility for all objects in the DLA sample. Actually there is an indication of a recent massive burst of star formation in at least the DLA at $z_{\mathrm{abs}}=3.025$ toward Q0347-3819 (Levshakov et al. 2002; Dessauges-Zavadsky et al. 2002b). The DLAs at $z \sim 2$ do not appear to be associated with a homogeneous population of galaxies, neither in terms of star formation history 
(morphological type) and age. This result is in agreement with the studies of DLAs at low redshifts $(z<1)$ where different types of galaxies have been shown to be the optical counterparts (e.g. Le Brun et al. 1997; Nestor et al. 2002). Our results confirm once again that simple plots of DLA abundances versus redshift cannot be used to estimate the overall metal evolution of the Universe, because the local parameters (as determined by the star formation history of the associated galaxy) would dominate the scatter in the measurements. We plan to extend our comparison of the DLA abundance patterns with chemical evolution models in 2004 to additional 5 objects for which the data have already been acquired.

Acknowledgements. The authors wish to extend special thanks to all people working at ESO/Paranal for the high quality of the UVES spectra obtained in service mode. We are grateful to G. Meynet for useful discussions and to A. M. Wolfe for interesting comments. We particularly thank C. Chiappini for many interesting advises and for her help to clarify important points. M.D.-Z. is supported by the Swiss National Funds. This work has partly been done during M.D.-Z. ESO studentship and has benefited of the support from the European Commission Research and Training Network "The Physics of the Intergalactic Medium".

\section{References}

Ballester, P., Modigliani, A., Boitquin, O., et al. 2000, Messenger, 101, 31

Bennett, C. L., Halpern, M., Hinshaw, G., et al. 2003, ApJS, 148, 1

Bergeson, S. D., \& Lawler, J. E. 1993a, ApJ, 408, 382

Bergeson, S. D., \& Lawler, J. E. 1993b, ApJ, 414, L137

Bergeson, S. D., Mullman, K. L., \& Lawler, J. E. 1994, ApJ, 435, L157

Bergeson, S. D., Mullman, K. L., Wickliffe, M. W., et al. 1996, ApJ, 464, 1044

Boissier, S., \& Prantzos, N. 1999, MNRAS, 307, 857

Bradamante, F., Matteucci, F., \& D'Ercole, A. 1998, A\&A, 337, 338

Calura, F., \& Matteucci, F. 2003, ApJ, 596, 734

Calura, F., Matteucci, F., \& Vladilo, G. 2003, MNRAS, 340, 59

Centurión, M., Bonifacio, P., Molaro, P., \& Vladilo, G. 2000, ApJ, 536, 540

Centurión, M., Molaro, P., Vladilo, G., et al. 2003, A\&A, 403, 55

Chen, Y. Q., Nissen, P. E., Zhao, G., Zhang, H. W., \& Benoni, T. 2000, A\&AS, 141, 491

Chiappini, C., Matteucci, F., \& Gratton, R. 1997, ApJ, 477, 765

Chiappini, C., Matteucci, F., Beers, T. C., \& Nomoto, K. 1999, ApJ, 515,226

Chiappini, C., Matteucci, F., \& Romano, D. 2001, ApJ, 554, 1044

Chiappini, C., Matteucci, F., \& Meynet, G. 2003, A\&A, 410, 257

Costa, R. D. D., Chiappini, C., Maciel, W. J., \& de Freitas Pacheco, J. A. 1996, A\&AS, 116, 249

Dessauges-Zavadsky, M., Prochaska, J. X., \& D’Odorico, S. 2002a, A\&A, 391, 801 (Paper I)

Dessauges-Zavadsky, M., D’Odorico, S., Levshakov, S. A., \& Molaro, P. 2002b, Chemical Enrichment of Intracluster and Intergalactic Medium, ed. R. Fusco-Femiano, \& F. Matteucci, ASP Conf. Ser., 253,137

D’Odorico, S., Cristiani, S., Dekker, H., et al. 2000, in SPIE 4005, Discoveries and Research Prospects from 8- to 10-Meter-Class Telescopes, ed. J. Bergeron, 121

D’Odorico, V., Petitjean, P., \& Cristiani, S. 2002, A\&A, 390, 13

Fan, X., Narayanan, V. K., Lupton, R. H., et al. 2001, AJ, 122, 2833
Fedchak, J. A., \& Lawler, J. E. 1999, ApJ, 523, 734

Fedchak, J. A., Wiese, L. M., \& Lawler, J. E. 2000, ApJ, 538, 773

Ferland, G. J., Korista, K. T., Verner, D. A., et al. 1998, PASP, 110, 1040

Fontana, A., \& Ballerster, P. 1995, Messenger, 80, 37

Goswami, A., \& Prantzos, N. 2000, A\&A, 359, 191

Gratton, R. G., \& Sneden, C. 1991, A\&A, 241, 501

Grevesse, N., \& Sauval, A. J. 1998, Space Sci. Rev., 85, 161

Hou, J. L., Boissier, S., \& Prantzos, N. 2001, A\&A, 370, 23

Howk, J. C., \& Sembach, K. R. 1999, ApJ, 523, L141

Howk, J. C., Savage, B. D., \& Fabian, D. 1999, ApJ, 525, 253

Izotov, Y. I., \& Thuan, T. X. 1999, ApJ, 511, 639

Izotov, Y. I., Shaerer, D., \& Charbonnel, C. 2001, ApJ, 549, 878

Jehin, E., Magain, P., Neuforge, C., et al. 1999, A\&A, 341, 241

Jenkins, E. B., Oegerle, W. R., Gry, C., et al. 2000, ApJ, 538, L81

Jimenez, R., Bowen, D. V., \& Matteucci, F. 1999, ApJ, 514, 83

Jura, M., \& York, D. G. 1978, ApJ, 219, 861

Kennicutt, R. C. Jr. 1983, ApJ, 272, 54

Kennicutt, R. C. Jr. 1998, ARA\&A, 36, 189

Kwitter, K. B., Henry, R. B. C., \& Milingo, J. B. 2003, PASP, 115, 80

Lanfranchi, G. A., \& Matteucci, F. 2003, MNRAS, 345, 71

Lanzetta, K. M., Noriaki, Y., Pascarelle, S., Chen, H.-W., \& Fernandez-Soto, A. 2002, ApJ, 570, 492

Le Brun, V., Bergeron, J., Boissé, P., \& Deharveng, J. M. 1997, A\&A, 321, 733

Ledoux, C., Srianand, R., \& Petitjean, P. 2002, A\&A, 392, 781

Levshakov, S. A., Dessauges-Zavadsky, M., D’Odorico, S., \& Molaro, P. 2002, ApJ, 565, 696

Lopez, S., Reimers, D., D’Odorico, S., \& Prochaska, J. X. 2002, A\&A, 385,778

Lopez, S., \& Ellison, S. L. 2003, A\&A, 403, 573

Lu, L., \& Wolfe, A. M. 1994, AJ, 108, 44

Lu, L., Sargent, W. L. W., Barlow, T. A., Churchill, C. W., \& Vogt, S. S. 1996, ApJS, 107, 475

Lu, L., Sargent, W. L. W., \& Barlow, T. A. 1998, AJ, 115, 55

Madau, P., Ferguson, H. C., Dickinson, M. E., et al. 1996, MNRAS, 283, 1388

Mathlin, G. P., Baker, A. C., Churches, D. K., \& Edmunds, M. G. 2001, MNRAS, 321, 743

Matteucci, F., \& Tornambé, A. 1987, A\&A, 185, 51

Matteucci, F., \& François, P. 1989, MNRAS, 239, 885

Matteucci, F., Raiteri, C. M., Busso, M., Gallino, R., \& Gratton, R. 1993, A\&A, 272, 421

Matteucci, F., Molaro, P., \& Vladilo, G. 1997, A\&A, 321, 45

Matteucci, F. 2001, The Chemical Evolution of the Galaxy, Astrophysics and Space Science Library, 253 (Dordrecht: Kluwer Academic Publisher)

McWilliam, A., Preston, G. W., Sneden, C., \& Searle, L. 1995, AJ, 109,2736

Meynet, G., \& Maeder, A. 2002, A\&A, 381, L25

Mishenina, T. V., Kovtyukh, V. V., Soubiran, C., Travaglio, C., \& Busso, M. 2002, A\&A, 396, 189

Molaro, P., Bonifacio, P., Centurión, M., et al. 2000, ApJ, 541, 54

Molaro, P., Levshakov, S. A., D’Odorico, S., Bonifacio, P., \& Centurión, M. 2001, ApJ, 549, 90

Morton, D. C. 1991, ApJS, 77, 119

Morton, D. C. 2000, ApJS, 130, 403

Morton, D. C. 2001, ApJS, 132, 411

Nestor, D. B., Rao, S. M., Turnshek, D. A., et al. 2002, in APS Conf. Ser., 254, Extragalactic Gas at Low Redshift, ed. J. S. Mulchaey, \& J. Stocke, 34

Nomoto, K., Hashimoto, M., Tsujimoto, T., et al. 1997a, Nucl. Phys. A, 616, 79 
Nomoto, K., Iwamoto, K., Nakasato, N. T., et al. 1997b, Nucl. Phys. A, 621,467

Nussbaumer, H., \& Storey, P. J. 1986, A\&AS, 64, 545

Outram, P. J., Chaffee, F. H., \& Carswell R. F. 1999, MNRAS, 310, 289

Pei, Y. C., Fall, S. M., \& Bechtold, J. 1991, ApJ, 378, 6

Pettini, M., Boksenberg, A., \& Hunstead, R. W. 1990, ApJ, 348, 48

Pettini, M., Smith, L. J., Hunstead, R. W., \& King, D. L. 1994, ApJ, 426, 79

Pettini, M., Ellison, S. L., Steidel, C. C., Shapley, A. E., \& Bowen, D. V. 2000, ApJ, 532, 65

Pettini, M., Shapley, A. E., Steidel, C. C., et al. 2001, ApJ, 554, 981

Pettini, M., Ellison, S. L., Bergeron, J., \& Petitjean, P. 2002, A\&A, 391,21

Pilyugin, L. S., Mollá, M., Ferrini, F., \& Vílchez, J. M. 2002, A\&A, 383,14

Prochaska, J. X., \& Wolfe, A. M. 1996, ApJ, 470, 403

Prochaska, J. X., \& Wolfe, A. M. 1998, ApJ, 507, 113

Prochaska, J. X., \& Wolfe, A. M. 1999, ApJS, 121, 369

Prochaska, J. X., Naumov, S. O., Carney, B. W., McWilliam, A., \& Wolfe, A. M. 2000, AJ, 120, 2513

Prochaska, J. X., Wolfe, A. M., Tytler, D., et al. 2001, ApJS, 137, 21

Prochaska, J. X, Henry, R. B. C., O’Meara, J. M., et al., 2002a, PASP, 114,933

Prochaska, J. X., Howk, J. C., O’Meara, J. M., et al. 2002b, ApJ, 571, 693

Prochaska, J. X., \& Wolfe, A. M. 2002, ApJ, 566, 68

Prochaska, J. X. 2003, ApJ, 582, 49

Prochaska, J. X., Howk, J. C., \& Wolfe, A. M. 2003, Nature, 423, 57

Raassen, A. J. J., \& Uylings, P. H. M. 1998, A\&A, 340, 300

Ryan, S. G., Norris, J. E., \& Beers, T. C. 1996, ApJ, 471, 254

Recchi, S., Matteucci, F., \& D'Ercole, A. 2001, MNRAS, 322, 800

Recchi, S., Matteucci, F., D’Ercole, A., \& Tosi, M. 2002, A\&A, 384, 799

Salpeter, E. E. 1955, ApJ, 121, 161

Sargent, W. L. W., Boksenbrg, A., \& Steidel, C. C. 1988, ApJS, 68, 539

Savage, B. D., \& Sembach, K. R. 1991, ApJ, 379, 245

Savage, B. D., \& Sembach, K. R. 1996, ARA\&A, 34, 279
Savaglio, S., Panagia, N., \& Stiavelli, M. 2000, in Cosmic Evolution and Galaxy Formation: Structure, Interactions, and Feedback, ed. J. Franco, L. Terlevich, O. Lopez-Cruz, \& I. Aretxaga, ASP Conf. Ser., 215,65

Scalo, J. M. 1986, FCPh, 11, 1

Schectman, R. M., Povolny, H. S., \& Curtis, L. J. 1998, ApJ, 504, 921

Sembach, K. R., Howk, J. C., Ryans, R. S. I., \& Keenan, F. P. 2000, ApJ, 528, 310

Sofia, U. J., \& Jenkins, E. B. 1998, ApJ, 499, 951

Steidel, C. C., Adelberger, K. L., Giavalisco, M., Dickinson, M., \& Pettini, M. 1999, ApJ, 519, 1

Stephens, A. 1999, AJ, 117, 1771

Storrie-Lombardi, L. J., \& Wolfe, A. M. 2000, ApJ, 543, 552

Storrie-Lombardi, L. J., Irwin, M. J., \& McMahon, R. G. 1996, MNRAS, 282, 207

Talbot, R. J. Jr., \& Arnett, D. W. 1974, ApJ, 190, 605

Theodosiou, C. E., \& Federman, S. R. 1999, ApJ, 527, 470

Tosi, M., Greggio, L., Marconi, G., \& Focardi, P. 1991, AJ, 102, 951

Tripp, T. M., Lu, L., \& Savage, B. D. 1996, ApJS, 102, 239

van den Hoeck, L. B., \& Groenwegen, M. A. T. 1997, A\&AS, 123, 305

Verner, D. A., Barthel, P. D., \& Tytler, D. 1994, A\&AS, 108, 287

Verner, D. A. 1996, At. Data Nucl. Data Tables, 64, 1

Viegas, S. M. 1995, MNRAS, 276, 268

Vladilo, G. 1998, ApJ, 493, 583

Vladilo, G., Centurión, M., Bonifacio, P., \& Howk, J. C. 2001, ApJ, 557,1007

Vladilo, G. 2002a, A\&A, 391, 407

Vladilo, G. 2002b, ApJ, 569, 295

Vladilo, G., Centurión, M., D’Odorico, V., \& Péroux, C. 2003, A\&A, 402, 487

Welty, D. E., Frisch, P. C., Sonneborn, G., \& York, D. G. 1999, ApJ, 512,636

Wiese, L. M., Fedchak, J. A., \& Lawler, J. E. 2001, ApJ, 547, 1178

Wolfe, A. M., Fan, X.-M., Tytler, D., et al. 1994, ApJ, 435, L101

Wolfe, A. M., \& Prochaska, J. X. 2000, ApJ, 545, 603

Wolfe, A. M., Prochaska, J. X., \& Gawiser, E. 2003a, ApJ, 593, 215

Wolfe, A. M., Gawiser, E., \& Prochaska, J. X. 2003b, ApJ, 593, 235

Woosley, S. E., \& Weaver, T. A. 1995, ApJS, 101, 181 